\begin{tabular}{|c|c|c|c|c|c|c|c|c|}
\hline \multicolumn{3}{|c|}{$\begin{array}{l}\text { 2. 10: (Receiving Organization) } \\
\text { WRAP } 1\end{array}$} & \multicolumn{2}{|c|}{$\begin{array}{l}\text { 3. From: (Originating Organization) } \\
\text { WRAP } 1\end{array}$} & \multicolumn{4}{|c|}{$\begin{aligned} \text { 4. Related EDT No.: } \\
\text { N/A }\end{aligned}$} \\
\hline \multicolumn{3}{|c|}{$\begin{array}{l}\text { 5. Proj./Prog./Dept./Div.: } \\
\text { W026/Solid Waste } \\
\text { Construction }\end{array}$} & \multicolumn{2}{|c|}{$\begin{array}{l}\text { 6. Design Authority/ Design Agent/Cog. } \\
\text { Engr.: } \\
\text { TL Watson }\end{array}$} & \multicolumn{4}{|c|}{$\begin{array}{l}\text { 7. Purchase order No.: } \\
\qquad N / A\end{array}$} \\
\hline \multirow{2}{*}{\multicolumn{5}{|c|}{$\begin{array}{l}\text { 8. Originator Remarks: } \\
\text { For release }\end{array}$}} & \multicolumn{4}{|c|}{$\begin{array}{l}\text { 9. Equip./Component No.: } \\
\text { N/A }\end{array}$} \\
\hline & & & & & \multicolumn{4}{|c|}{$\begin{array}{c}\text { 10. System/8ldy./Facility: } \\
\mathrm{N} / \mathrm{A}\end{array}$} \\
\hline \multirow{3}{*}{\multicolumn{3}{|c|}{ 11. Receiver Renarks: }} & \multirow{3}{*}{\multicolumn{2}{|c|}{ asel ine Document? [] res $[X]$ No }} & \multicolumn{4}{|c|}{$\begin{array}{l}\text { 12. Major Assm. Dwg. No.: } \\
\text { N/A }\end{array}$} \\
\hline & & & & & \multicolumn{4}{|c|}{$\begin{array}{l}\text { 13. Permit/Perinit Application No.: } \\
N / A\end{array}$} \\
\hline & & & & & \multicolumn{4}{|c|}{$\begin{array}{l}\text { 14. Required Response Date: } \\
\text { N/A }\end{array}$} \\
\hline \multicolumn{2}{|l|}{15.} & \multicolumn{3}{|c|}{ DATA TRANSMITTED } & (F) & (G) & $(\mathrm{H})$ & (I) \\
\hline $\begin{array}{l}\text { (A) } \\
\text { Item } \\
\text { No. }\end{array}$ & (B) Document/Drawing No. & $\begin{array}{l}\text { (C) } \\
\text { sheat } \\
\text { No. }\end{array}$ & $\begin{array}{l}\text { (D) } \\
\text { Rev. } \\
\text { No. }\end{array}$ & $\begin{array}{l}\text { (E) Title or Description of Data } \\
\text { Transmitted }\end{array}$ & $\begin{array}{l}\text { Approval } \\
\text { Desig- } \\
\text { nator }\end{array}$ & $\begin{array}{l}\text { Reaton } \\
\text { for } \\
\text { Trans. } \\
\text { mit:al }\end{array}$ & $\begin{array}{l}\text { Origi- } \\
\text { nator } \\
\text { Dispo- } \\
\text { sition }\end{array}$ & $\begin{array}{l}\text { Receiv- } \\
\text { ef } \\
\text { Dispo- } \\
\text { sition }\end{array}$ \\
\hline 1 & WHC-SD-W026-ATP-013 & & 0 & Fire Alarm System Test Plan & Q & 2 & 1 & \\
\hline$>2$ & WHC-SD-W026-ATR-013 & & 0 & Fire Alarm System Iest Report & a & 2 & 1 & \\
\hline & & & & & & & & \\
\hline & & & & & & & & \\
\hline & & & & & & & & \\
\hline
\end{tabular}

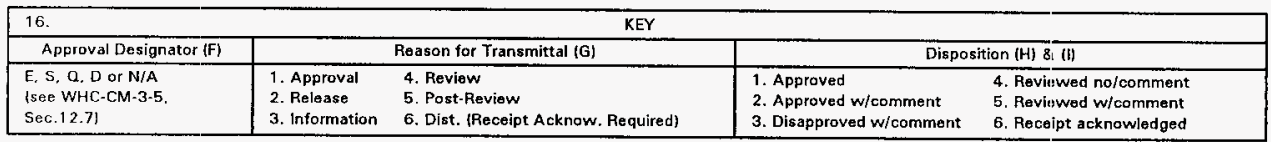

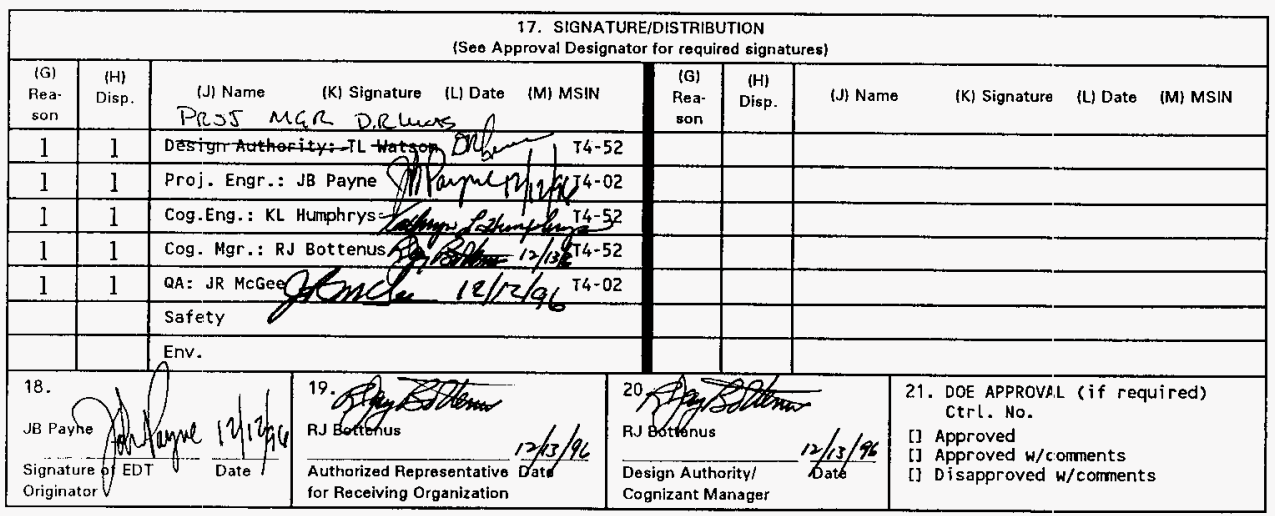



WHC-SD-W026-ATR-013, Rev. 0

\section{W-026, Acceptance Test Report Fire Alarm System (Submittal \# 1571.1)}

TL Watson

Westinghouse Hanford, Richland, WA 99352

U.S. Department of Energy Contract DE-AC06-87RL10930

$\begin{array}{lll}\text { EDT/ECN: } & 161635 & \text { UC: } \\ \text { Org Code: } & 04 E 00 & \text { Charge Code: P1JF18 } \\ \text { B\&R Code: } & 39 E W 3130020 & \text { Tota1 Pages: } 92\end{array}$

Key Words: ATR, WRAP 1, Fire Alarm, Fire Detection, Fire Suppression

Abstract: This Acceptance Test Report was written by 3-D Protection Systems, Inc., and PCL Construction. WRAP 1 Facility Engineering, Solid Waste Fire Safety, Kaiser Acceptance Inspector and Hanford Fire Department personnel witnessed this test. All exceptions were resolved. The resolutions are attached. Contractor's Material and Test Certificates are attached. Results from Solid Waste Industrial Hygiene sound level surveys are also included.

IRADEMARK DISCLAIMER. Reference herein to any specific commercial product, process, or service by trade name, trademark, manufacturer, or otherwise, does not necessarily constitute or imply its endorsement, recommendation, or favoring by the United States Government or any agency thereof or its contractors or subcontractors.

Printed in the United States of America. To obtain copies of this document, contact: INC/BCS Document Control Services, P.O. Box 1970, Mailstop H6-08, Richland WA 99352, Phone (509) 372-2420; Fax (509) 376-4989.
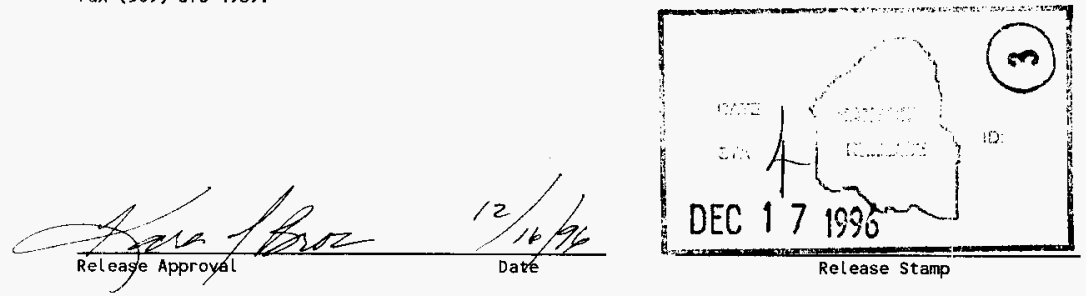

Approved for Public Release 

KH-5366-ATP REV. 4

ACCEPTANCE TEST PROCEDURE KH-5366-ATP REV. 4 DATE 4/26/96

TEST TITLE FIRE ALARM SYSTEM

IOCATIOH WASTE RECEIVING \& PROCESSING FACILITY

QROJECT NUMBER $\underline{W-026}(F-5918-P)$

PEOJECT TITLE WASTE RECEIVING E PROCESSING FACILITY

Prepared by

3-D PROTECTION SYSTEMS, INC.

FOI ICF RAISER ENGINEERS

Contract $\mathrm{KH}-5366$

PROCEDURE APRROVAL

ICF KIISER HENEORD COMPANY (ICE KU)
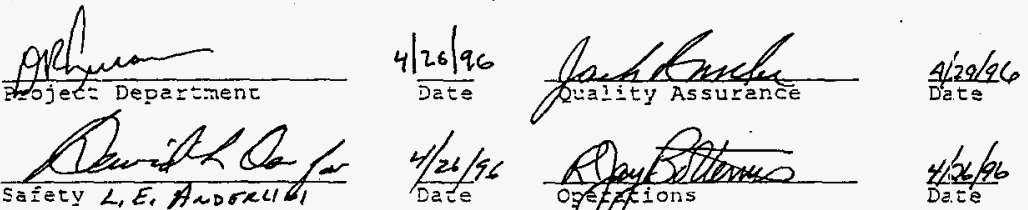

$4 / 26 / 46$

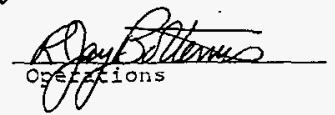

$\frac{4 / 26 / 96}{D a .0 e}$

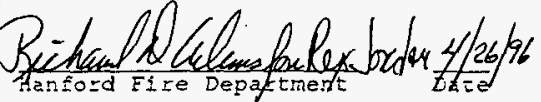




$$
\text { WHC-SD-W026-ATR-013, Rev. } 0 \text { Page } 02
$$$$
\text { KH-5366-ATP REV. } 4
$$

EXECUTION AND TEST APRROVAL

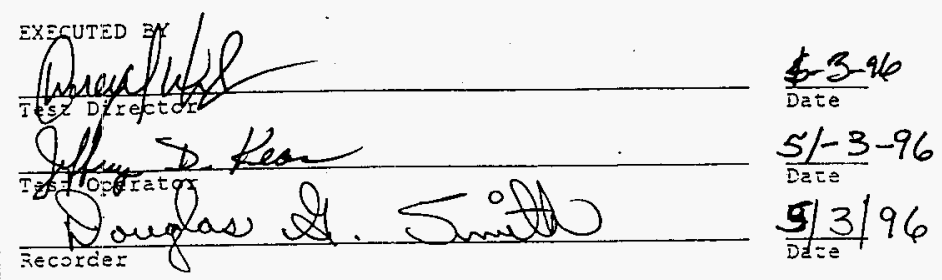

WITES SES

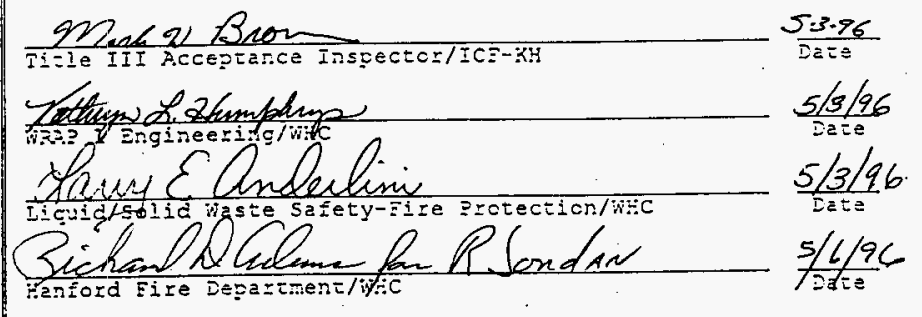

TEST APDROVAL AND A.CCEPTALICE
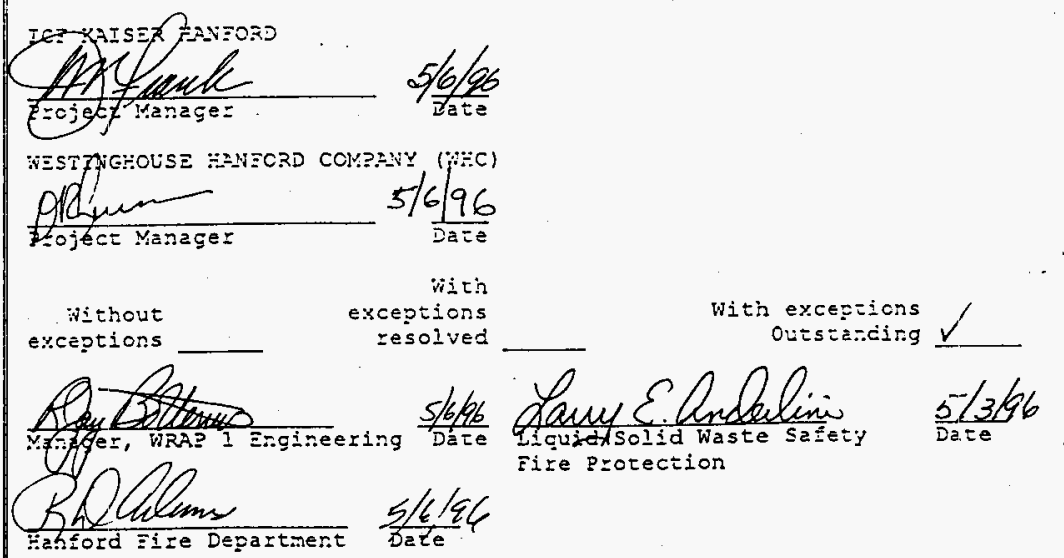

Ifoidetsolid waste satety

$\frac{5 / 366}{5 a=e}$

Fafford Eire Departmert Date

ii 
TABLE OF CONTENTS

1.0 PURPOSE. . . . . . . . . . . . . . . . . . . . . . . . . . . . . 1

2.0 REFERENCES.............................. 1

2.1 DRAUINES . . . . . . . . . . . . . . . . . . . . . . . . . 1

2.2 SPECIEICATIONS . . . . . . . . . . . .............. . . . . . . .

2.3 ENGINEERING CHANGE NOTZCES (ECN). . . . . . . . . . . . . . . . 1

3.0 RESPONSIBIIITIES . . . . . . . . . . . . . . . . . . . . . . . . . .

3.1 GENEKZL . . . . . . . . . . . . . . . . . . . . 2

3.2 TEST DIRECTOR . . . . . . . . . . . . . . . . . . . . . .2

3.3 WITLESSES . . . . . . . . . . . . . . . . . . . . . . . . .3

3.4 RECCRDER............................ 3

3.5 TEST CEERTOR. . . . . . . . . . . . . . . . . 4

4.0 CEANGE CONTROL . . . . . . . . . . . . . . . . . . . . . . . . . . 4

5.0 Execotion . . . . . . . . . . . . . . . . . . . . . . . . . . . . . .4 4

5.1 OCCU?AEICNAI SAEETY AND HEATH. . . . . . . . . . . . . . . . . .5

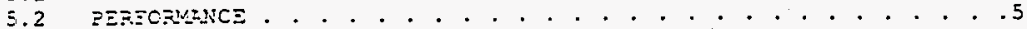

6.0 EXCEPTIONS . . . . . . . . . . . . . . . . . . . . . . . . 5

6.1 GENEAAL. . . . . . . . . . . . . . . . . . . . . . . . . 5

E.3 RETEST/RESOLUTION . . . . . . . . . . . . . . . . . . . . . . . .

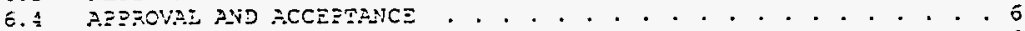

6.5 DISRRIEUIICU . . . . . . . . . . . . . . . . . . . . . . . . 6

7.0 IREZEQUISTES AND EQUIERENT FEQUIRED . . . . . . . . . . . . . . . . . 7

7.1 PEIREQUISITES . . . . . . . . . . . . . . . . . . . .

7.2 EQUIZWENT AND INSTRUEZTS . . . . . . . . . . . . . . 8

7.3 R.3EKEVIATIONS . . . . . . . . . . . . . . . . . . . .

8.0 FIRE ALAEY SYSIZM TESTS. . . . . . . . . . . . . . . . . . . . .9

8.1 DEEZZRZTION . . . . . . . . . . . . . . . . . . 9

8.2 PCNER TENNSEER. . . . . . . . . . . . . . . . . . . . 10

2.3 CIECUIT SUEERVISION . . . . . . . . . . . . . . . . . . . . . . 11

8.4 3EIE/SIROEZ SYZASS TEST. . . . . . . . . . . . . . . . . . . 17

8.5 SHUIDCWN SYPASS TEST . . . . . . . . . . . . . . . . . .18

9.6 PIDES FRID SUPERVISORY TEST . . . . . . . . . . . . . . . . . . . 19

9.7 HIGE/ICN PRESSURE SWITC: TEST . . . . . . . . . . . . . . . . 31

8.8 PIV AND TAMPER SWITCH TEST . . . . . . . . . . . . . . . .32

8.9. DRY CHEMICPI SYSTEY RUD SHUTDON TEST . . . . . . . . . . . . . 33

8.10 ZUXILIARY ECNER SUPQIY SLPESVISION TEST . . . . . . . . . . . . 36

8.11 S:HUTDONN REIAY PCWER SU?ERVISICN . . . . . . . . . . . . . . . .37

3.12 PIZVT MZNAGENEITT SYSMEM TEST . . . . . . . . . . . . . . . . . . 37

8.13 E.ATIERY DRAIN DONN. . . . . . . . . . . . . . . . . . . . . . . .38

9.0 SECURE EROM ACCEPTANCE TEST. . . . . . . . . . . . . . . . . . 41

A.ZZENDIX A: INSTALIER CHECKLIST

MPPENDIX $3:$ EXCEPTICN S:EETS 


\section{KH-5366-ATP REV. 4 \\ ACCEPTANCE TEST PROCEDDRE \\ FOR TES 2336- W \\ WASTE RECEIVING AND PROCESSING FACILITY \\ MODOLE I (WRAP 1)}

\subsection{PURPOSE}

This Acceptance Test Procedure (AT2) has been prepared to cemonstrate that the Eire Erotection systen functions as recuired by the project criteria.

The cest results will by issued as an accentence test report (ATR) a:teer all testing is complete. The rumber assigned to the ATR is Mr-53Ga-ATR.

\subsection{REEERENCES}

2.1 DRAVIINGS

3-D PROTECTION SYSTEXS, INC. E-5\$18-Z SHEETS 1,2,3,4, 5, \&6

2.2 SEECIEICATIONS

$16720,15300,15370,16050,16110,16120$, IND $15 I 52$

2.3 EUGINEERING CHNNE NOIICES 


\section{KH-5366-ATP REV. 4}

3 RESPONSIEIUTES

II GENERAL

Eech company cr organization garicipating in this ATP will designate ferscrnel to ossumg the resporsiojübes and dutes as denned herein for thetr ascectiva rales. The decignees stial tecome familier with this ATP and tha systems irvolved to ine extent thet tiey cal fertiont disir assigned duties.

\subsection{TEST OIRETIOR}

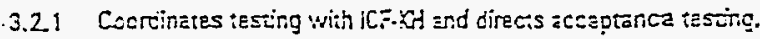

3.22 Ac:s as ileison cetween ibe farticifants in acceptanca terting.

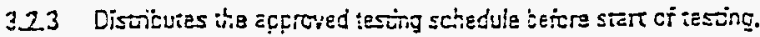

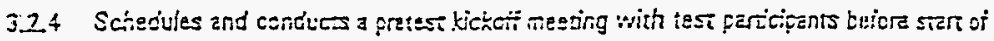
tascing when recessary.

3.25 Nowīes the persons pericming and wiosescing the test 2 wering ciays beicre tha stert criessing.

\subsection{Schedules a dry rin winen necessary.}

3.27 Nomines concerried paries when a chanç is nade in the testing sciedule.

32.1 Takes necessery icsion io ciesr excepicns io the test

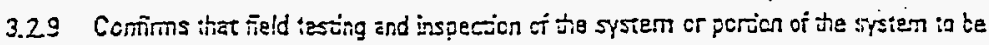
ierted has kean cencileted.

3 -10 Steps any astwich, in hisher judgmen may cause denaçe to the sysiem unti the froolem has ben resclved.

3.2.11 Afier verifying there is no aciversa impaca, nay ahter the sequenez in whicit systems or suisysians are tesied.

3.2.12 Eosures that required enviconmental conditions are maintained

3.213 If a test is to ta suspenced for a period of time, ensures that the srstem is iert in a saie micde. 
3.214 Eeicio resiaring susçencied :ast, reveriñes ite est frerequisites.

3.2.15 Initiates ECNs to dociment required chançes io the ATP.

3.216 Reviews secorded data, ciscreșancies, and excepticns.

3.2.17 Obtains information cr chançes hecessary to dear or resohe objections diring tie pericmence citive tert

3.2.13 Signs Exection and Test Af̣civel paça wren ast has besn feritrmed.

32.19 Signs Erçọtion iorm when excspicion heș ien resoived.

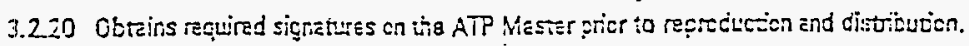

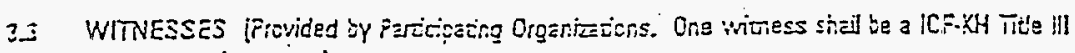
aceapticnce insçecior.|

\subsubsection{Witress ith test:}

3.32 Review resuits ct testing.

3.3.3 Assigt the Test Direcer Winen requestad.

3.3.4 Siçn Ëection and Texi Aç̣oral façe when iest has been fericmed.

3.3.5 Sicn Excsption fim when extepion has isen resclved.

\subsection{AECORDEA}

3.4.1 Prepares a Fiald eapy from itia ATP Masier.

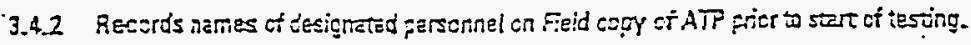

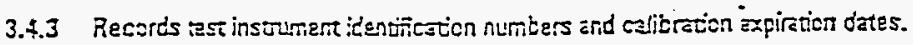

3.4.4 Inibiels and dates each iest steg on the fieid copy as it is conpletad, next to the siep number, or cn a daa sheet wrien provided. Records Tesa Deta. On data sheets where itere is not reon icr sceh tie initizl and ciate. dats may te entered at batton of column.

3.4.5 Fecerds objections and excopticrs on an Excepticn form. Transiars intomation in ink or type 10 an Encepcion icm masier. Uses adcrional Exception forms as needed. 
3.4.6 Signs Erecution and Tess Ạç̣toval paçe when iest has been periomed.

3.4.j After iast is finished, assignis aloha numeric paoge numbers is edded deta shegrs and Erception forms. Recaris page numcers in the Table ct Contents.

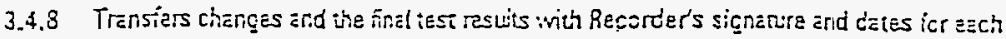

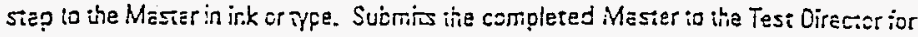

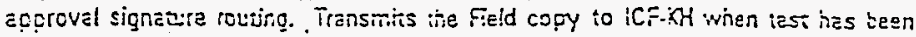
acseg̣ied and excêpritions cioseci.out.

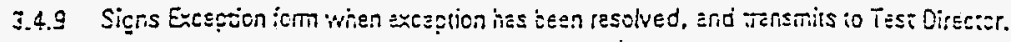

\section{S.E TEST OFEFATOR}

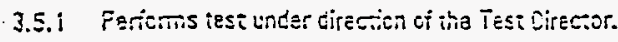

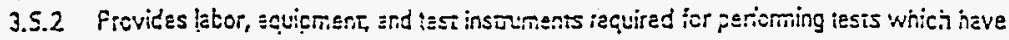
net been cesignated as teing provided by outers.

3.5.3 Reguests in writing form ICF.KM tiose servicss. materiais. or squipment that have Eeen desicnated as beirg stectied by otters.

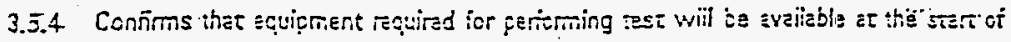
iesing.

3.5.E Signs ihs Execution ard Test Aforoval gags. .

4 CHANEE CONIROL

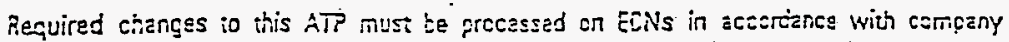
procedures. if a need for chença is jiscevered in tie sourse of nunirg the iest, the iert shall be sicpped until the ECN is aecroved. Heivever, this coss not prevent tis ninning ci anchter porion of the tert unatiaced by the change.

$\Xi \quad$ EKECUTION

5.1 OCCUPATIONAL SAFETY AND HEALTH

Inorviduais shail camy-out their assigned work in a saie manner to protect themsetves and cthers from undus hazerds and to prevent damaģe to property and ervironment. Faciily line managers shall assure the safety of acsivides within their aress to grevent injury, preperty demaģe. or interniption of operavion. Pericrmencz of test accivities stiell ahways includi safery and health aspects. 


\section{KH-5366-ATP REV. 4}

\section{\$2 PEAFORMANCE}

\subsection{Use ICE-RH concirted cspr ci this ATP.}

5.2.2 Conciuc ter meeting iesfonsïbitity requirements of Secion 3 of this ATP.

5.23 Feriom test in accordence with sieps in Secsicn(s) \& (9, etc) of the ATP.

5.2.7 When test has been completed obtain signantes of test enecuticners and withesses.

5.2.5 Sucrit ita testrepon to iCf-Ah ier afproval. $=$ -

NOTE I: At this poirr. tha "proedure" becenes a "ieport".

NOTE 2 Thera may be sacaptions outstanding.

\subsubsection{Ciear excegtions and submir :o iC:-KH for actroval.}

NOTE: Idesily, the clesrad arceptions woudd be submitied with the report. Cesceinate with iCF-KH as to allewable delay benween iest complesion and submitials.

\section{E ERCETIONS}

\section{S.I " GEVERAL}

Exceptions to the required iest sesuis are sequenciaily numbered and iecoried on incividual Excapoion foms. Tris enables cesa-by-ese resoivioi and efproval ot ach excaurion

Erors/excsprions in the ATP itseff sireiliNoT be procesed as test excepticns (see Secsion 4).

\subsection{FECDRDING}

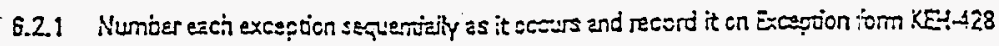
(samp̣le ap̣ended), or an aççrved sisetiture.

6.22 Enter nems and org̣anization oi objecsing farty for escì exception.

6.2.3 Enter planned acsion to resolva each exceprion when such detemination is made.

\subsection{REIEST/RESOLUTION}

Record tie acjion tcken to resolve each excapjon. Action tekeri may not be the sarre as planned acsion. 


\section{KH-5366-ATP REV. 4}

6.3.1 When action izken resulis in an accepteble retest, sign and cata Retest Execiuion and Acesptancs secion ci the Exception fom.

6.3.2 Wh:en action teken does not invoho an acoppable retert soike out tie Retest Erecurion and Acceprance section of the Exception form.

6.4. AFPROVAL AND ACCETTANCE

ICF-XH provides inal approval and acraprance ci exceptions by checking one of the icilowing on Encepoion iorm:

5.4.1 Reter Approved and Acested: Applicatle when Retest Evecrion asd Acteprence secion is comp̣ieted.

6.4.2 Exception Á.coepied-Ass-is. Requires deziled explanavion.

6.4.3 Cuther Requires dstailed exp̣lanation.

ICF-XH signs and dates tha Excepoion form and oticins theñ cuscomer apgroval, as required.

5.5 OISTRIBUTICN

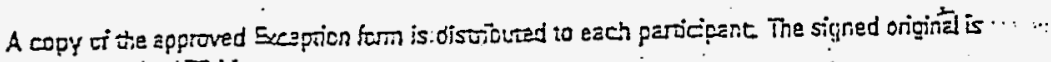
aracied to the ATP Masier. 


\section{KH-5365-ATP REV. 4}

\subsection{FREREQUISTIES AND EQUIPMENT REQUIRED}

\subsection{RREREQUISITES}

The following conditicns shall exist at the start of the testing for that portion of tie system being tested.

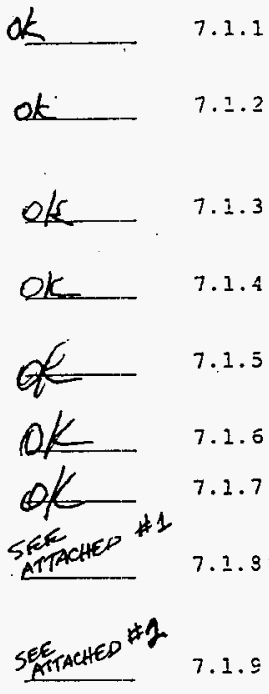
systems have been irspected for ccmpliance with
constructicn documents

keference dccuments inclucing this AT? have been verizied for correct revision numier ard outstanding ECNs.

Apterdix A for this AT? tas been satisfactorily ccmpleted and signed by installer and witness.

The REAR panel has zean verified coezational and placed in service with alarms bypassec.

$\vec{F}=C Z / R E A R$ zone and $t=0$ bile concuctors have been terminated.

Poner is available.

Test instuments have a valid calibration stano attaches.

Cortractor's Vateriai * Test Certificate for j- Loveground Eiping has iesn compleced in accordance with NEZA 13, as axplicable.

Contractor's Materied \& Test Certificete for U-cierground Piping has been completed in accorciance with NEZA 24, as apolicable. 
WHC-SD-W026-ATR-013, Rev. D Page 11

KH-5366-ATP REV. 4

7.2 EQDIPMENT AND INSTROMENTS

All ecuipment and instruments are to be supplied by the Test operator, Instalier, or Coatractor.

7.2.1 Portable Volt-chmezers (VOM): Rarge 0-50 volts DC and $0-1$ megohn.

Instrument No.80719018 Calizzation Expiration Date $4 / 30 / 97$

7.2.2 Eortable imeter: kange $0-10$ amperes.

$\phi$

Insizunent No. 63850299 caliszaicn Expiraticn Date $4 / 30 / 97$

7.2.3 ?crable Lecitel Vets=: Range 0-20c̈b

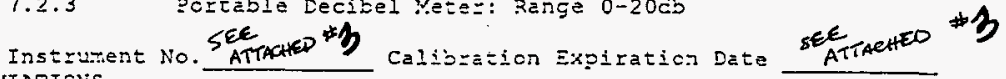

7.3 : ESREVIATIONS

EC. - Ergineering Change Notice

EAC? - Zi:

REAR - Radio Fire Alarm Reporting Eox

ATE - Pccepiace Test proceduse

ATK - Acceptance Test Report

¿ZD - Fanford Eire Depaztrent

ICE-3:- Integrated Constructicn Zorces, Kaiss= Hanford

IED - íghi Emiz=ir:g Diocie

ZXs - Ziant Management system

Wif - Mestingrouse Hanfozd Congary

vCY - Volt-Chrrister

cat. of eal.

Tagk BEL-IC96-10 model 107 luke calrepont $x 10$ Toge BE- IC 96-1l model 153 Becteman-BEL96

8 of 41 


\section{KH-5366-ATP REV. 4}

\subsection{FIRE AIARM SYSTEM TESTS}

This AT? will test the Pyrotronics system fire alarm control panel (EAC?), and inceriaces with tre REAR, alarm/stieervisory initiating devices, and alarm incicating appliances.

8.1 FREPFRATICN

QK 8.1 .1 Verify prezecuisites of subsection 7.1 have been net.
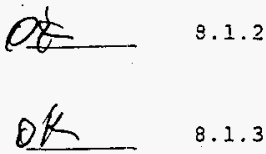

$8 \cdot 1 \cdot 3$
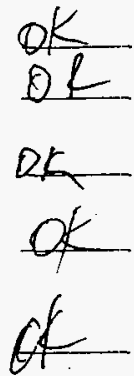

8.1 .6

8.1 .7

8.1 .4

8.1 .5

3.2 .8
Recuest the :anford Eire Departhent dispatcher bypass ali sigrals Erom tre TEAR 2730 and ackrowlecige ali sucervisory ard alarms received.

Announce to perscnnel in building that a fire alazm test is in progress; evacuaticr. is rot recuizec.

Install RE:R door tamper switch overzice device. Vezify bot: FACD cors aze keyed to open with CAI 60 $\mathrm{K} \in \mathrm{Y}$.

Depress laxp test sinizsi on FAC? ard verify all iEDs cone ch.

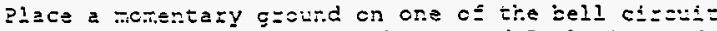

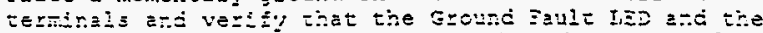
System Izciale IED come cN. 32 \& 35 To

Place a nctentary grcund on cne of ine zone ingut cizcuiz tez-.inals ard verify that the Ground Faul IDD and sysien Trouble IED come ON. 


\subsection{POWER TRANSFER}

This test will verify autcmatic trensfer to battery tpon AC pcher interruption and turn to rormal upon restoration of ic pcwer.

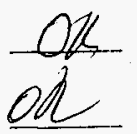

9.2.1 At powar suply parel, open FaCP stpply breakez to interrupt $A C$ power to FACP.

8.2 .2

Verify EAC? AC powez $I E D$ is $O F F$ and $t=c$ uble buzzer is OL_ 8.2 .3 sotidikg. Silence trouble buzzer.

ok

8.2.4

At poive supply parel, open REJR sLpply breake= to irterrupt $=C$ power to REAR.

Verify 2730 "Iccal ?arel Trouble" ressege follonad after approximately $\leqq 0$ second delay by "Conn Tril REAR" Inessage received by dispatchez.

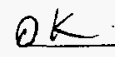

8.2 .5

Rese ŻC? and REAR.

$1 / \mathcal{K}_{1}$

8.2 .6

Place a momentary jumpe= betreen Terminals 2 and 5 , on Moćule $2 D$ of F.C.C?.

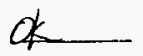

8. 2.7

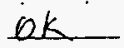

$3.2 \cdot 3$

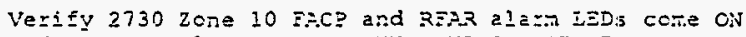
ard systens alarn gongs sOUHD AND SIRCEES Fi_ASE.

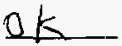

8.2 .9

Verify 2730 "Zone 10 ‥1arm" messace received by dispatcier.

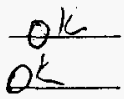

8.2 .10

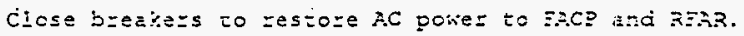

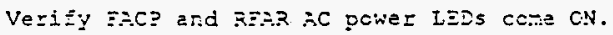

8.2.11

Reset Eac? end $\mathbf{s}=2.3$. 


\section{KH-5366-ATP REV. 4}

\subsection{CIRCOIT SUPERVISION}

This test will verify RERR Zone switch position and that both the gong and the input zone wiring are supervised.

8.3.1 RFAR Switch And CiIcuit sugervision

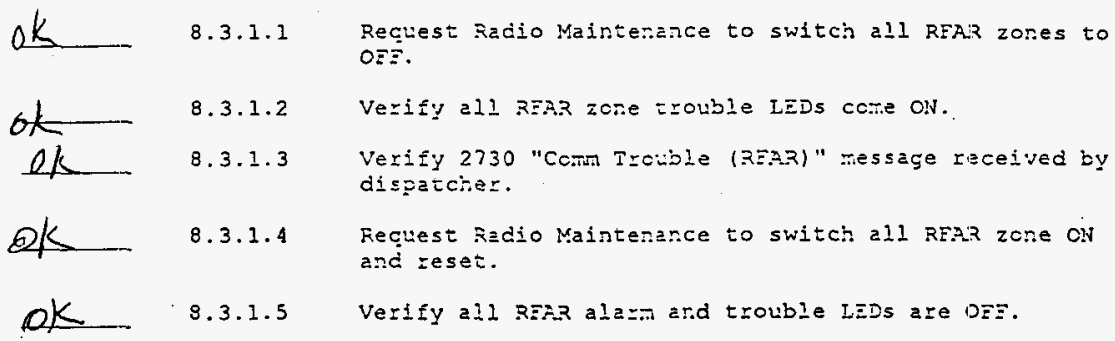




\section{KH-5366-ATP REV. 4}

\subsubsection{EACP ZONE SUPERVISION}

Record the following steps for the iters shown on Data sheet 8.3.2.

8.3.2.1 Disconnect the end of line (E.0.1) cevice for the indicated zone.

8.3.2.2. Verify E.:C? system and zone/audible circuit trouble IEDs are CiN.

8.3.2.3 Vezify 2730 "Iccal Earel Trouble" nessage fo: RE:AR zeceived by cispatcher.

8.3:2.4 Recorrect the E.0.I. Cevice. Reset E:CP.

8.3.2.3 Vezify E:C? system, zcne/audible cifcuit, and REAR troulie IEDs are OFE.

\begin{tabular}{|c|c|c|c|c|c|c|}
\hline . & \multicolumn{6}{|c|}{ DATA S:EET 8.3 .2} \\
\hline & & & INEU & TSDI & IE 20 & \\
\hline S?E? & EERECKY/VERIEY & 21 & 22 & 23 & 24 & 25 \\
\hline 8.3 .2 .1 & Discoriect E.0.I. Sevice & 依 & ok & ok & $0 k$ & OK \\
\hline 8.3 .2 .2 & Trolible LEDs a $=0$ ON & & $\Delta k$ & $8 K$ & ok & $\alpha<$ \\
\hline 9.3 .2 .3 & Trouble ressage zecEived & ok & ok & ok & $\overline{2 k}$ & $O F$ \\
\hline 8.3 .2 .4 & Recctiect E.0.I, cievice & DK & $\theta k$ & $D K$ & $a k$ & ok \\
\hline $8 . \overline{3} \cdot 2.5$ & T=OLible IEDS aze CSZ & & & of & $o k$ & ok \\
\hline
\end{tabular}


WHC-SD-W026-ATR-013, Rev. 0 Page 16

KH-5366-ATP REV. 4

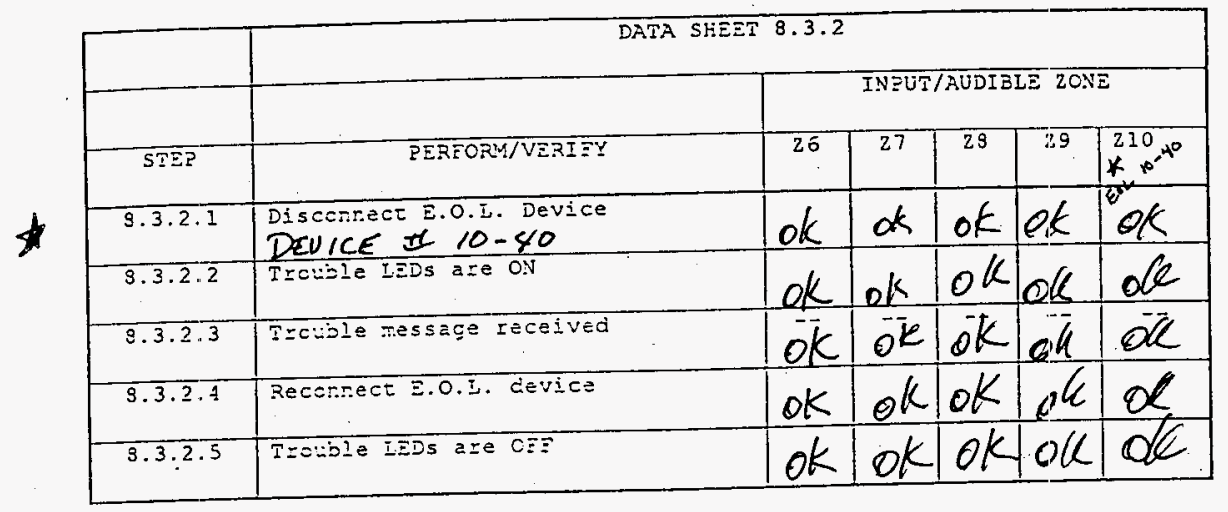

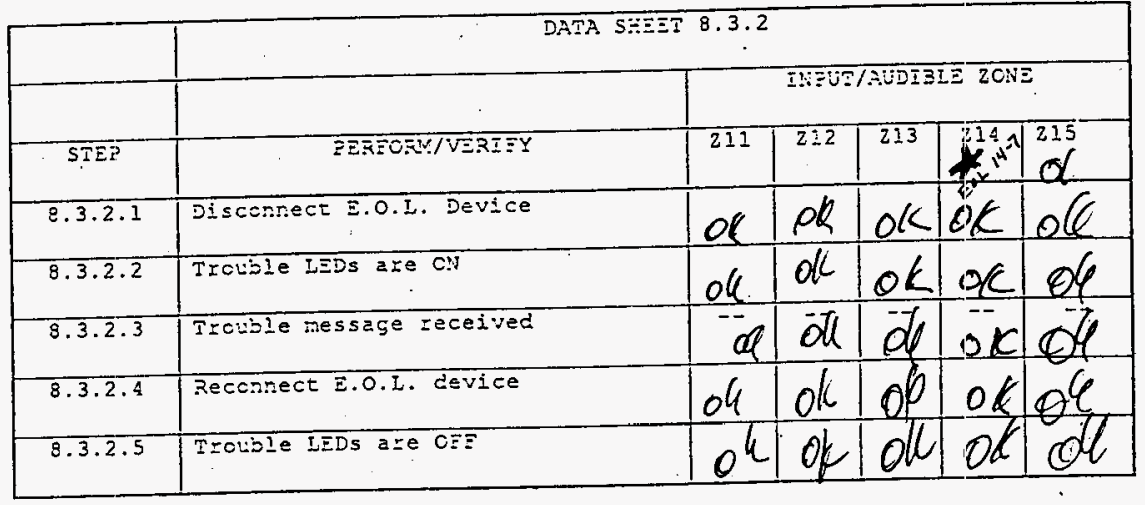

* Exception 4

13 of 41 
KH-5366-ATP REV. 4

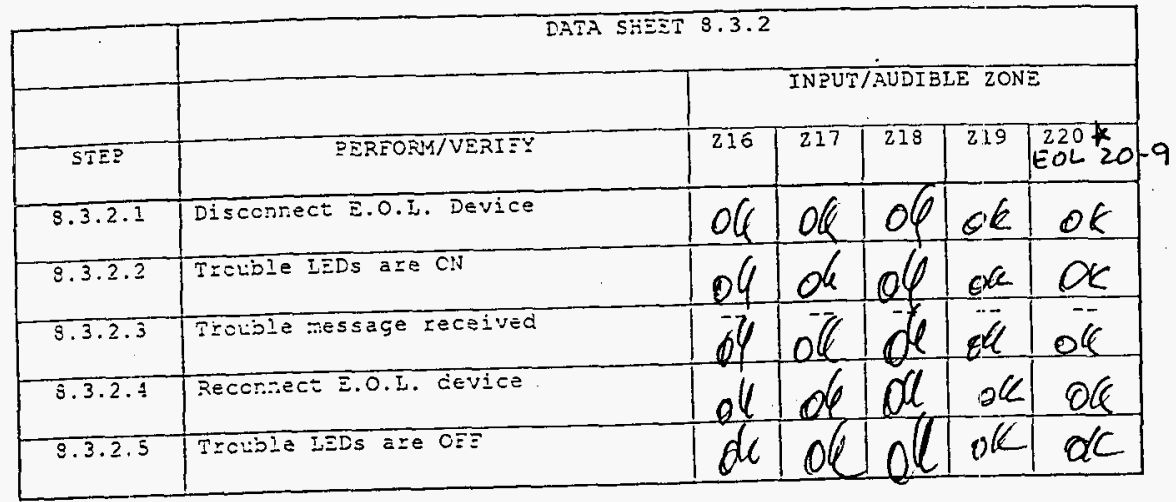

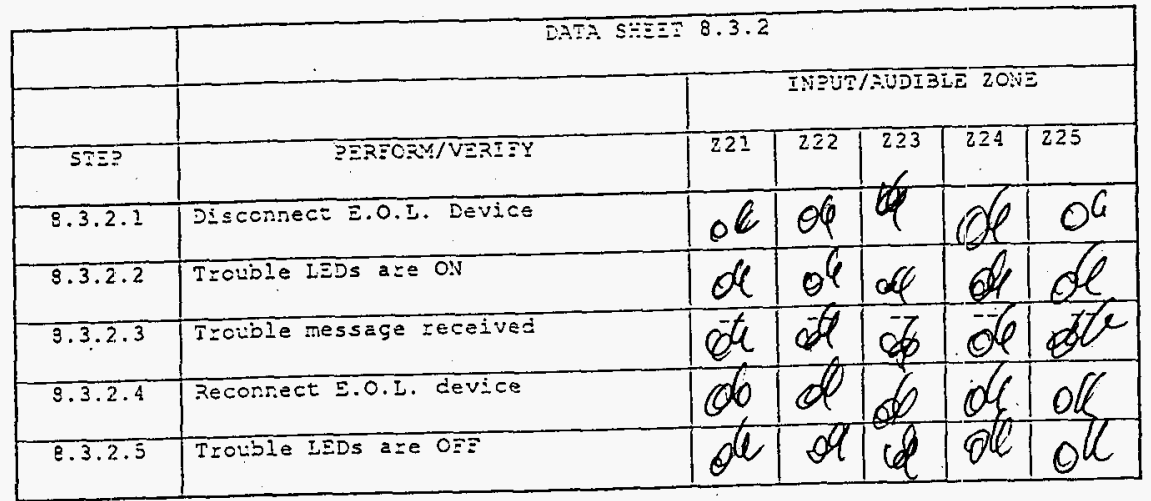

K EXCEPTION \#1

14 of 41 
WHC-SD-WO26-ATR-013, Rev. 0 Page 18

KH-5366-ATP REV. 4

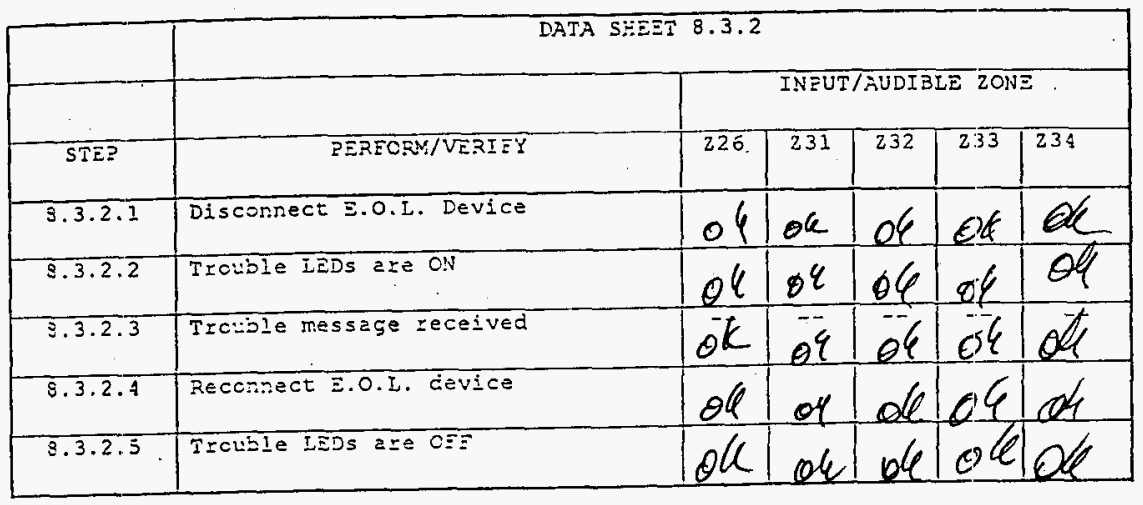

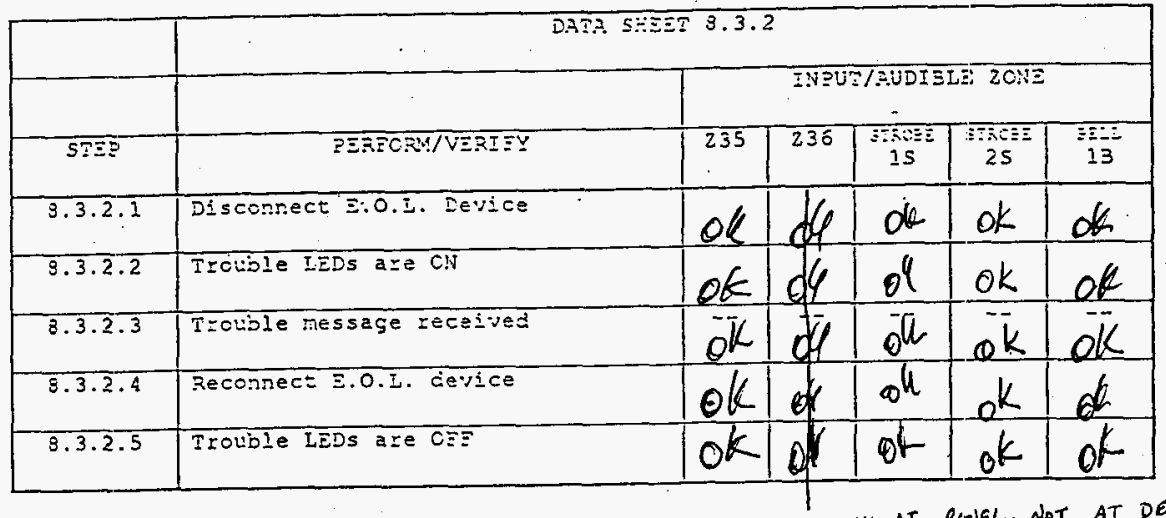

D.D THLS AT PENEL, NOT aT DENIE ExcelTiOA $\# 8$

15 of 41 
KH-5366-ATP REV. 4

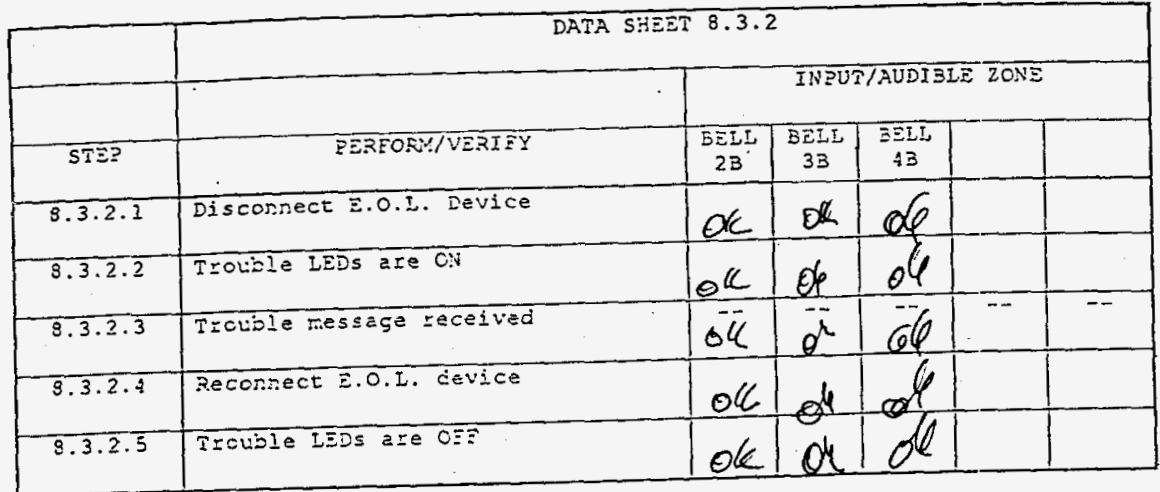




$$
\text { - }
$$


WHC-SD-W026-ATR-013, Rev. 0 Page 20

\section{KH-5366-ATP REV. 4}

8.4 BELL/STROBE BYPASS TEST

This test will verify the supervision and performance of the bell/bypass switch.

ok

8.4 .0

Jypass tells and strobes.

8. 4.1

Verify systen trouble IED is old.

Dasign will EXCETION

Bypuss

oL

\$. A. 2

Verify trouble nessage raceived by dispatcher.

8. $4 \cdot 3$

Verify trouble sigral on giant management system.

ok

9. $2 \cdot 4$

short across iezminals 2 a:c 5 on module $2 D$.

8.4. 5

Verify EAC? zone elarn IED is ON.

Q4e

8.4 .6

Vezizy FAC? systen alarn izD is ON.

3. 4.7

Verify bells ard strobes are OFF.

8.4 .8

Verify REAR zcre alerm zeceived by dispatcier.

B. 4.9

Return byoess switch to rozmal gosition.

B. 4.10

Verify cpezaticn cf the belis arc stzojes.

9.4 .11

ackrowlecge system alara.

8. 1.12

Return bypass sinitch to rormal positich.

3. 4.13

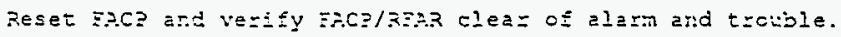




\section{KH-5366-ATP REV. 4}

\subsection{SHOTDOWN BYPASS TEST}

This test will verify the supervision and perfomance of the bypass switch.

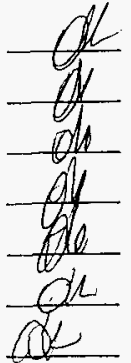

8.5 .0

8.5 .1

8.5 .2

8.5 .3

8.5 .4

8.5 .5

8.5 .6

8. 5.7

8. $5 \cdot 8$

8.5 .9

$8 \cdot 5 \cdot 10$

$8.5 \cdot 11$

8.5 .12

$8.5 \cdot 13$
Bypass shutdown function.

Verify systen trouble IED is ON.

Verify trouble nessage received by dispatcher.

Verify trouble signal on plant management system.

$\div$

short across teminals 2 and 5 on module $3 c$.

Verify EACP zone alarn LED is ON.

Verify FACP system alarn LED is ON.

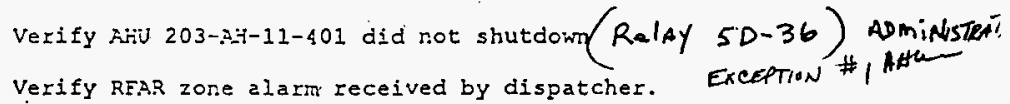

Return the by-pess switch to the nome? position.

Verify Shutdown function.

Acknowledge system alam.

Retura bypass switch to nomal position.

Reset FAC? and verify EACP/REAR clear of alam and trouble. 


\section{KH-5366-ATP REV. 4}

\subsection{AIARM AND SUPERVISORY TEST}

This test will verify the initiation device capability of all elarm and supervisory input devices. Record steps 8.6.1 through 8.6.6 on Data Sheet 8.6 for each device

is ised.
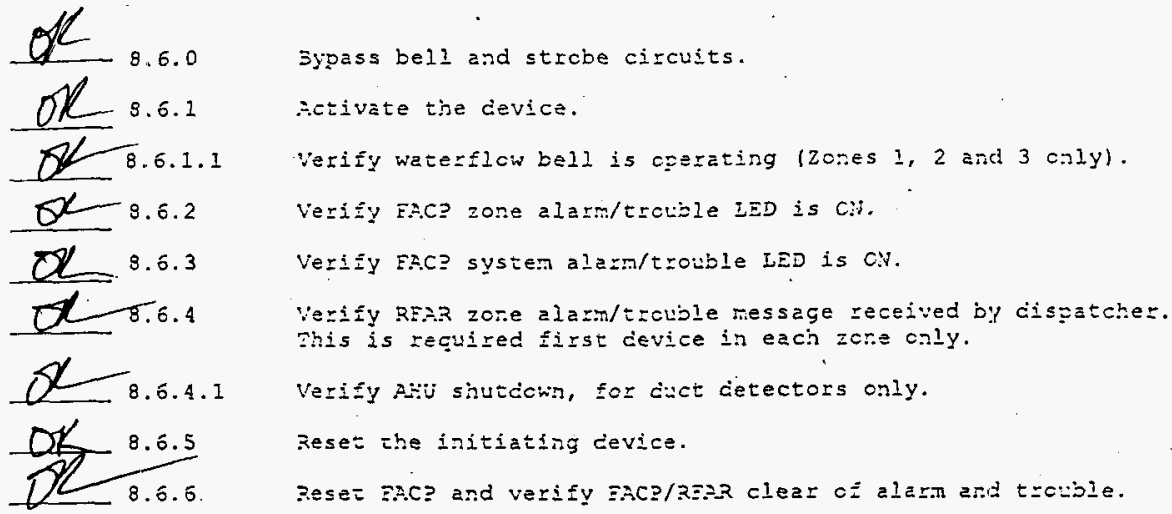
KH-5366-ATP REV. 4

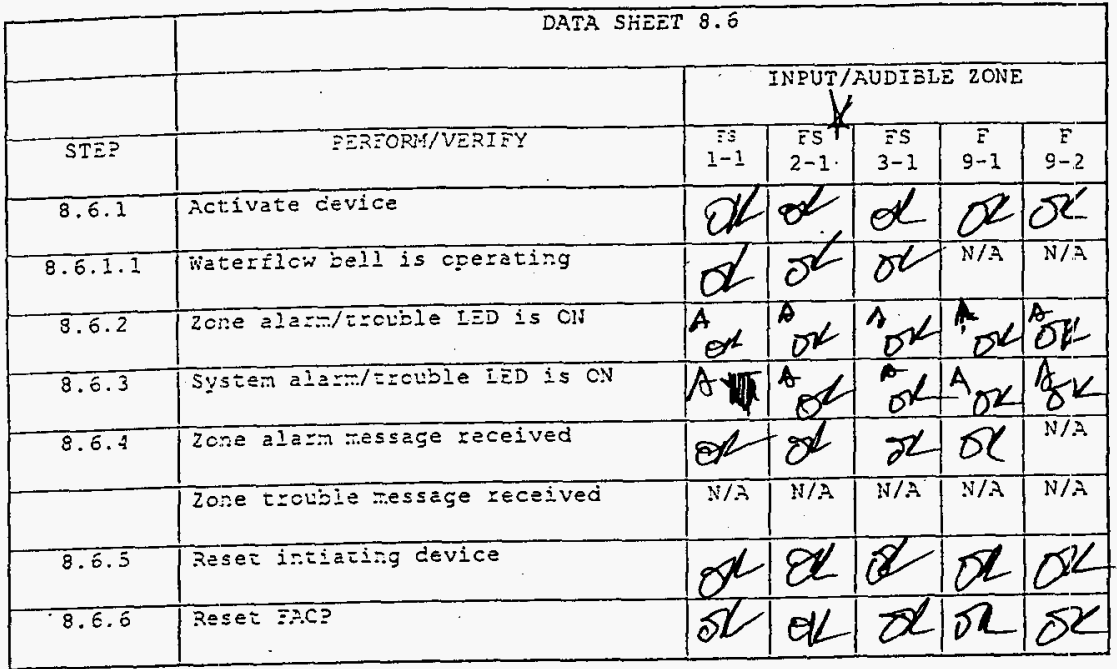

4 Exception \#3 


\begin{tabular}{|c|c|c|c|c|c|c|}
\hline 28 & 20 & 28 & $\infty$ & & $\sum \partial \forall Z$ Zәsәg & $9 \cdot 9 \cdot 8$ \\
\hline$x Q$ & 20 & $x$ & $\not 3$ & & 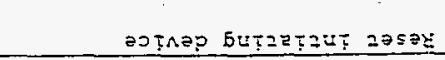 & $5 \cdot 9 \cdot 8$ \\
\hline$\forall / N$ & $\checkmark / \mathrm{Ai}$ & $\because / \aleph$ & $\forall / N$ & $F / \ddot{i}$ & 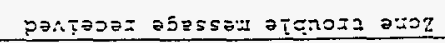 & \\
\hline$\ddot{z} / \mathbf{N}$ & $\forall / M$ & $\forall / R$ & $E / N$ & $Y / i$ & 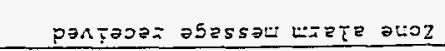 & $=9 \cdot 8$ \\
\hline & 20 & $\pi 0$ & & & 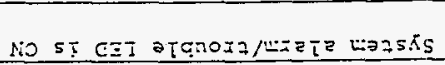 & $\varepsilon \cdot 9 \cdot 8$ \\
\hline & 20 & 25 & & & 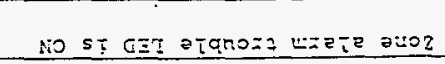 & $2 \cdot 9 \cdot 8$ \\
\hline & $\rightarrow$ & $x$ & & & 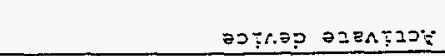 & {$[\% .8$} \\
\hline $\begin{array}{c}Z \cdot \tau-\dot{6} \\
I\end{array}$ & $\begin{array}{c}\tau I-\sigma \\
I\end{array}$ & $\begin{array}{c}0 \longleftarrow-6 \\
\dot{3}\end{array}$ & $\begin{array}{c}6-5 \\
4\end{array}$ & $a-5$ & 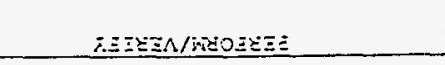 & $\varepsilon \equiv \Sigma S$ \\
\hline & $\mathrm{ENOZ}=$ & EzGR & InEN & & & \\
\hline & & & & 0.5 & 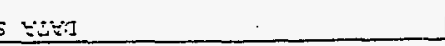 & \\
\hline
\end{tabular}

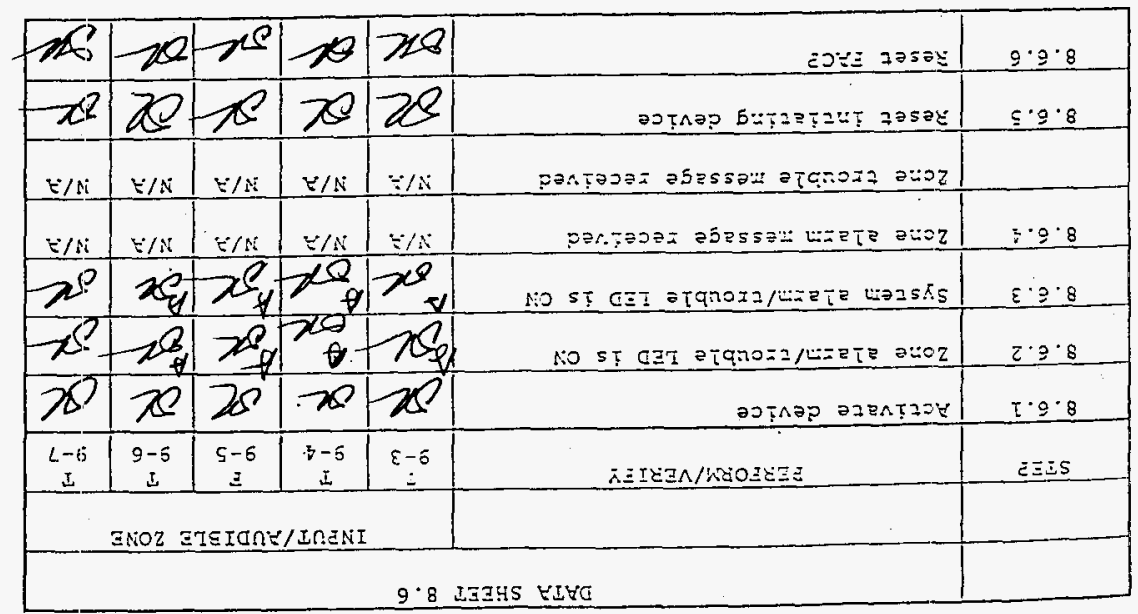

$\downarrow \wedge \exists y \quad d \perp \forall-99 \varepsilon s-H X$ 
KH-5366-ATP REV. 4

\begin{tabular}{|c|c|c|c|c|c|c|}
\hline & \multicolumn{6}{|c|}{ DATA SHEET 8.6} \\
\hline & & & INEUT & AUDISI & $\Xi \mathrm{ZONE}$ & \\
\hline STE? & PERTORM/VERIEY & $9-13$ & $\begin{array}{c}T \\
g-1 A\end{array}$ & $\begin{array}{c}T \\
9-15\end{array}$ & $5-16$ & $10-1$ \\
\hline 8.6 .1 & Activate cevice & $x$ & $\sigma^{2}$ & $\alpha$ & & $i$ \\
\hline 8.5 .2 & Zone alarm/trouble IED is of & & $\infty$ & & & $\mathscr{x}$ \\
\hline 3.5 .3 & System alarm/tzocible ISO is C:N & & 2 & & & 20 \\
\hline $8 . \overline{6} . \frac{1}{4}$ & Zore alazm thessage received & $N / 1$ & $N / A$ & iv/ & $N / A$ & $3 \pi$ \\
\hline & Zcre trouble ressage received & $N / A$ & $N / A$ & $\mathrm{~N} / \mathrm{A}$ & $\mathrm{N} / \mathrm{A}$ & II/A \\
\hline 8.6 .5 & Reser intiaring cievice & $\delta^{2}$ & $\delta^{\prime}$ & $O C$ & $\delta x$ & $w 2$ \\
\hline 8.6 .6 & Resat EAC? & i火 & 5 & $8 x^{2}$ & $0 \mathbb{Z}$ & $x$ \\
\hline
\end{tabular}

\begin{tabular}{|c|c|c|c|c|c|c|}
\hline & \multicolumn{6}{|c|}{ DATA SEEET 8.6 } \\
\hline & & \multicolumn{5}{|c|}{ INOUT/ALDIELE 2CHE } \\
\hline STE? & PEREOSMTVERIE? & $10-2$ & $\begin{array}{c}7 \\
10-3\end{array}$ & $\frac{3}{20-4}$ & $\begin{array}{c}T \\
10-5\end{array}$ & $\begin{array}{c}T \\
1.0-6\end{array}$ \\
\hline 8.6 .1 & Activate cevice & & & & \&h & $\partial$ \\
\hline 8.6 .2 & Zone alarn/tzoLizle IED is oN & & & & De & $8 x$ \\
\hline 3.6 .3 & System alarm/trouble IED is CN & & & & $\pi$ & $2 x$ \\
\hline 8.6 .4 & Zone alarm tressage received & IN/A & $\mathrm{N} / \mathrm{A}$ & $N / A$ & $N / 2$ & $N / A$ \\
\hline & 2one tzouble message recuived & $N / 2$ & N/A & $N / \therefore$ & $\mathrm{N} / \mathrm{A}$ & N/A \\
\hline 8.6 .5 & Reset intiating cevice & & 2 & 5 & 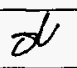 & st \\
\hline 8.5 .6 & Reset $\overline{\text { FAC? }}$ & & 2 & $o^{d}$ & $\delta x$ & 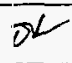 \\
\hline
\end{tabular}


KH-5366-ATP REV. 4

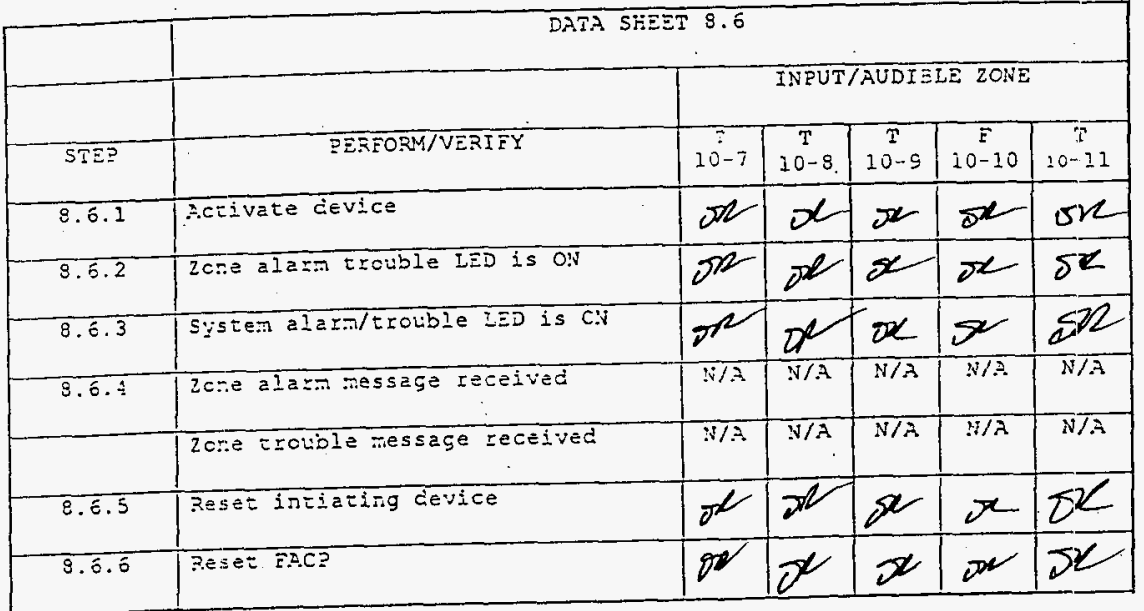

\begin{tabular}{|c|c|c|c|c|c|c|}
\hline & & & INPUT & FUIJI & $20 N E$ & \\
\hline & EERECER/1)ERIE' & $10-12$ & $10-13$ & $\frac{T}{10-34}$ & $10-15$ & $10^{2}-16$ \\
\hline 8.5 .1 & Activate cevice & 52 & $D^{2}$ & & $D$ & $\infty$ \\
\hline 8.5 .2 & Zcre alarm/Erclible IED is CN & & & & ox & $\infty$ \\
\hline 9.6 .3 & Sysiem alerm/trouble IED is oN & $\Delta 2$ & & & 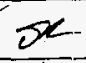 & 8 \\
\hline 8.6 .4 & Zcre alarm message zecéved & $\because i / A$ & N/A & $N / A$ & $\mathrm{~N} / \mathrm{A}$ & $N / A$ \\
\hline & Zcre trcliele message =eceived & $\mathrm{N} / \mathrm{A}$ & $N / A$ & $N / A$ & $\bar{N} / \mathrm{A}$ & $\mathrm{N} / \mathrm{A}$ \\
\hline 8.6 .5 & Resec intiating device & $\mathscr{D}$ & $s^{2}$ & & $\mathscr{2}$ & 22 \\
\hline 8.6 .6 & Reset FAC? & O & Der & $\bar{O}$ & $2 z^{2}$ & $x$ \\
\hline
\end{tabular}


WHC-SD-W026-ATR-013, Rev. 0 Page 27

KH-5366-ATP REV. 4

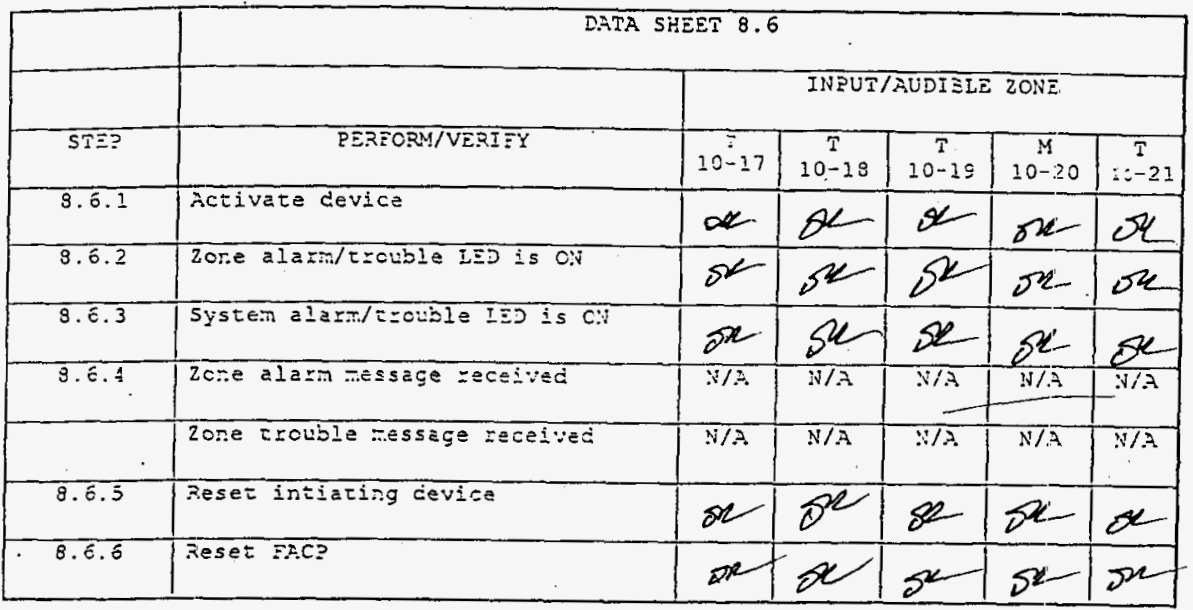

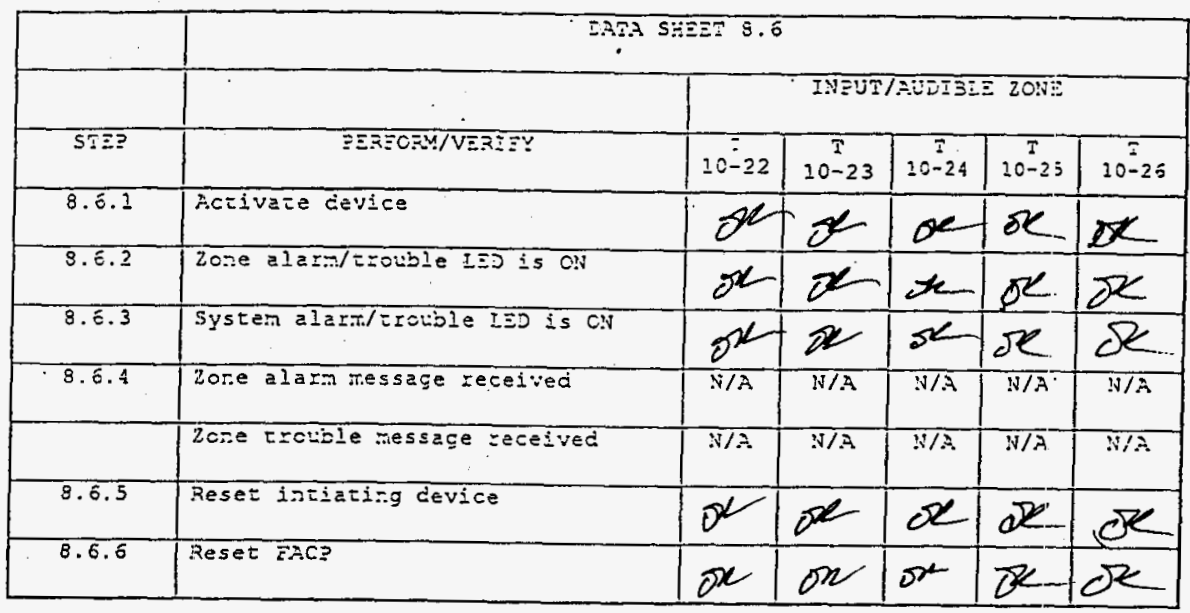

24 of 41 
KH-5366-ATP REV, 4

\begin{tabular}{|c|c|c|c|c|c|c|}
\hline & \multicolumn{6}{|c|}{ DATA SHEET 8.6} \\
\hline & & \multicolumn{5}{|c|}{ INFUT/AUDISIE 2ONE } \\
\hline STE? & ZEREOEM/VERIFY & $10-27$ & $\frac{T}{10-28}$ & $\frac{T}{10-29}$ & $\frac{T}{10-30}$ & $\begin{array}{c}7 \\
\therefore-31\end{array}$ \\
\hline 8.6 .1 & Activate cevice & of & $\mathbb{E}$ & $\sigma^{2}$ & De & o \\
\hline 8.5 .2 & 2 cone alarm trcuble lED is of & $\partial$ & 2 & $y$ & $\infty$ & $p^{2}$ \\
\hline 3.6 .3 & System alara/trouble lED is $0 . N$ & $\delta x$ & $\delta^{2}$ & $\delta$ & se & $\sigma^{2}$ \\
\hline $3.6 . \frac{1}{4}$ & $20: 3$ elarm fessace received & $\$ 1 / 3$ & $\mathrm{~N} / \mathrm{A}$ & $\overline{i / A}$ & 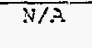 & $3 / \bar{A}$ \\
\hline & Zone erouble nessage received & N/S & $\mathrm{N} / \mathrm{A}$ & N/S & $\mathrm{N} / \mathrm{A}$ & $\$ N / A$ \\
\hline 8.6 .5 & Seset intiating cerice & $s$ & $x$ & \& & $\partial x$ & 82. \\
\hline 8.6.6 & Seset FRC? & 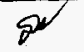 & $\rho^{2}$ & De & $0 \times 2$ & $\Delta x$ \\
\hline
\end{tabular}

\begin{tabular}{|c|c|c|c|c|c|c|}
\hline & \multicolumn{6}{|c|}{ SAT3 SHEET 3.6} \\
\hline & & & INPUT & AUSIES & $20 \mathrm{NE}$ & \\
\hline STE? & EERECYM/VERIEY & $10-52$ & $\begin{array}{c}T \\
10-33\end{array}$ & $\begin{array}{c}2 \\
10-34\end{array}$ & $\frac{7}{20^{2}-35}$ & $10-35$ \\
\hline 9.6 .1 & Activaze device & $\delta$ & $\infty$ & $\sigma^{2}$ & $x$ & $\infty$ \\
\hline 8.6 .2 & Zone alarm/t=cuble ted is oN & De & $D 2$ & $s$ & $\infty$ & $\dot{\delta}$ \\
\hline 8.6 .3 & System alare/crouble IED is $\mathrm{ON}$ & If & $O 2$ & 8 & $D^{2}$ & Se \\
\hline 8.6 .4 & Zore elarn ressage rectived & N/A & $N / A$ & $\mathbb{Y} / \mathrm{A}$ & $\mathrm{N} / \mathrm{A}-$ & N/A \\
\hline & Zore trouble ressage zeceived & $1 / \%$ & N/A & $N / A$ & $N / A$ & N/A \\
\hline 8.6 .5 & Reset intiating device & $D$ & 82 & $S$ & $x^{2}$ & $\infty$ \\
\hline 8.6 .6 & Reset Fac? & $b^{2}$ & $0^{2}$ & $2 x$ & $x^{2}$ & De \\
\hline
\end{tabular}


KH-5366-ATP REV. 4

\begin{tabular}{|c|c|c|c|c|c|c|}
\hline & \multicolumn{6}{|c|}{ DATA SRIEET 3.6} \\
\hline & & & INEUT & IADIBI & ZONE & \\
\hline STE? & ZEREORN/VERI I YY & $\begin{array}{c}=37 \\
10-37\end{array}$ & $\begin{array}{c}\mathrm{T} \\
10-38\end{array}$ & $\begin{array}{c}T \\
10-39\end{array}$ & $\begin{array}{c}\mathrm{T} \\
10-40\end{array}$ & $\therefore \begin{array}{c}\mathrm{T} \\
\therefore-41\end{array}$ \\
\hline 8.6 .1 & Acrivare device & $\infty$ & or & ok & $\delta^{3}$ & $x$ \\
\hline 8.6 .2 & Zo:e ala=:/ercuble IID is CN & $x$ & $x$ & $s$ & $\pi$ & 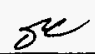 \\
\hline 3.6 .3 & System alazm/tzouble iED is oN & $D E$ & or & $x$ & $\pi^{\prime}$ & $\sqrt{2}$ \\
\hline 8.5 .4 & Zone alarn ressage received & 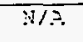 & IN/A & $\mathrm{N} / \mathrm{A}$ & N/A & $: 1 / \mathrm{A}$ \\
\hline . & Zene croulis lessage zeceived & $\because / A$ & N/A & N/A & N/A & $N / A$ \\
\hline 8.6 .5 & Reset irfieting device & $x$ & $\mathscr{Q}$ & $2 x$ & $x$ & $d x$ \\
\hline 8.6 .5 & SESET FAC? & & se & $x$ & $\delta$ & 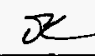 \\
\hline
\end{tabular}

\begin{tabular}{|c|c|c|c|c|}
\hline & \multicolumn{4}{|c|}{ DATA SHEET 3.6} \\
\hline & & 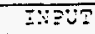 & AUDIJI & $20 \% 5$ \\
\hline STE? & EEREORM/VERIFY & $10-2$ & $\begin{array}{c}\bar{z} \\
10-43 .\end{array}$ & 13 \\
\hline 8.6 .1 & Activare cevice & $\delta$ & of & ot \\
\hline 8.5 .2 & Zore alazen/r=cible lED is $\mathrm{CN}$ & SE & $D^{\mathscr{N}}$ & $\sigma^{2}$ \\
\hline 8.6 .3 & Zcre ales:- /t=ouble LED is o:d & $\ddot{2}$ & $S^{2}$ & $\delta$ \\
\hline 8.6 .4 & Zore alarn ressage received & $\mathrm{SAO}$ & $N / A$ & $x$ \\
\hline & Zone trotile thessage received & $\$ / 2$ & N/A & $N / A$ \\
\hline 8.5 .5 & Reset intiating device & $D x$ & De & 82 \\
\hline 8.6 .5 & Reset FAC? & & $\infty$ & $O^{X}$ \\
\hline
\end{tabular}


KH-5366-ATP REV. 4

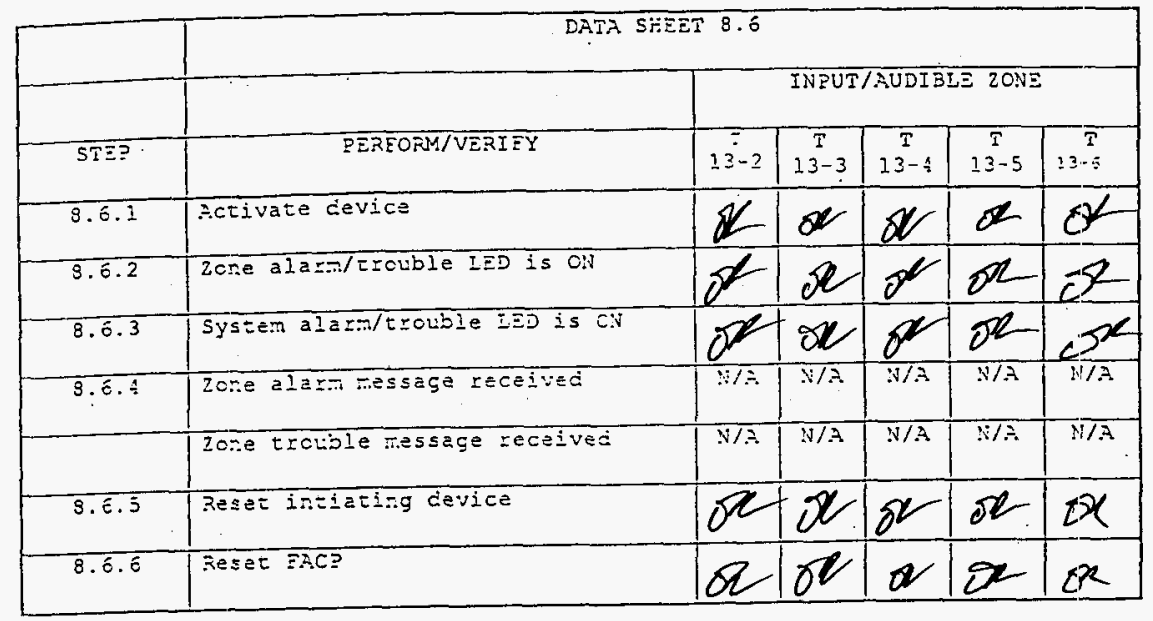

\begin{tabular}{|c|c|c|c|c|c|c|}
\hline & . & & & & & \\
\hline & & $13-7$ & $\begin{array}{c}I \\
13-8\end{array}$ & $\bar{T}$ & $\underset{14-1}{2}$ & $\frac{7}{14-2}$ \\
\hline 3.6. 1 & Acsivate device & $\mathscr{D}$ & & $\varnothing$ & Od & $O 2$ \\
\hline 9.6 .2 & Zone alarn/frouble LED is CN & $x$ & $\widetilde{D}$ & & & 22 \\
\hline 8.6 .3 & System alam/trolble IED is CN & & & & & 82 \\
\hline 3.6 .4 & 2ore alazer ressage received & $\therefore A$ & $N / A$ & $N / A$ & & N/A \\
\hline & 2cre trcliole message received & $1 / \therefore$ & $N / A$ & $N / A$ & 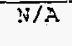 & N/A \\
\hline 8.6 .5 & Reset intiating device & $\sigma^{2}$ & $\sigma 2$ & & $\alpha$ & DR \\
\hline 3.6 .6 & Reset EACP & & $O^{2}$ & & ot & \\
\hline
\end{tabular}




$$
\text { WHC-SD-W026-ATR-013, Rev. } 0 \text { Page } 31
$$

KH-5366-ATP REV. 4

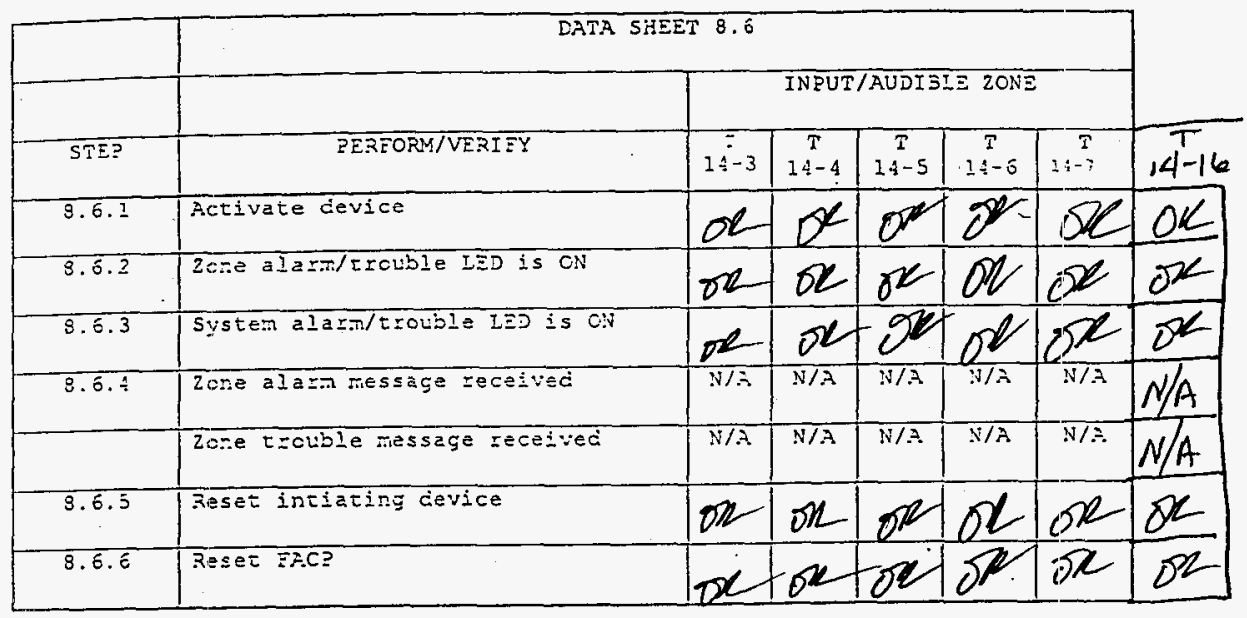

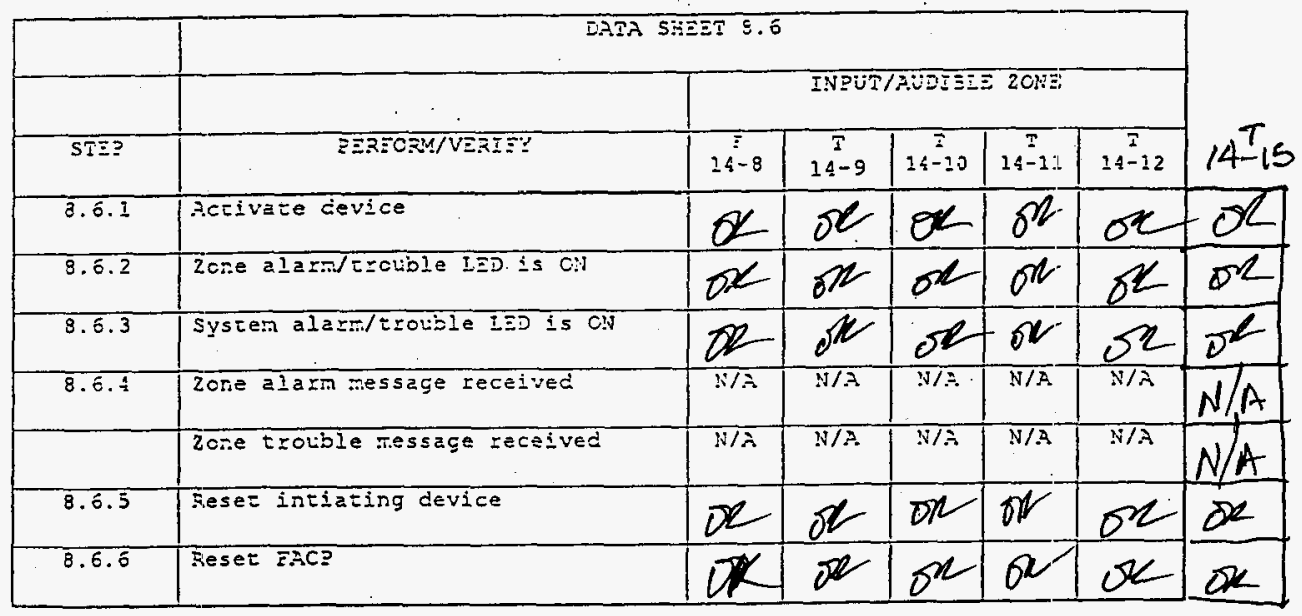

28 of 41 


$$
\text { WHC-SD-W026-ATR-013, Rev. O Page } 32
$$

KH-5366-ATP REV. 4

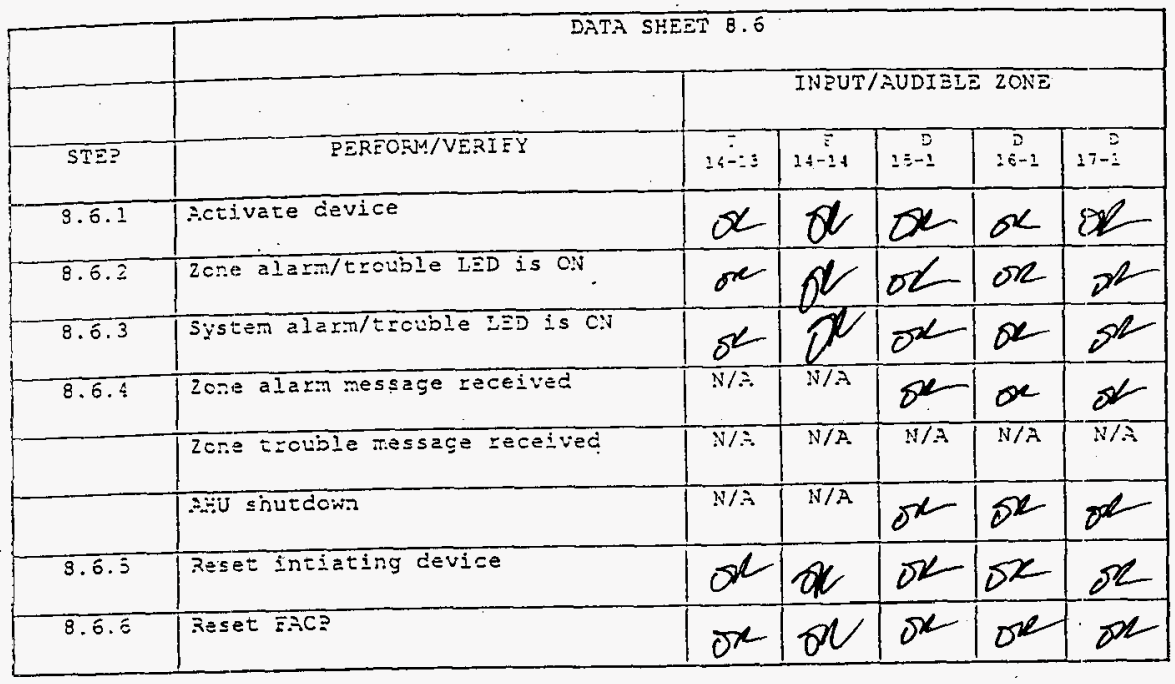

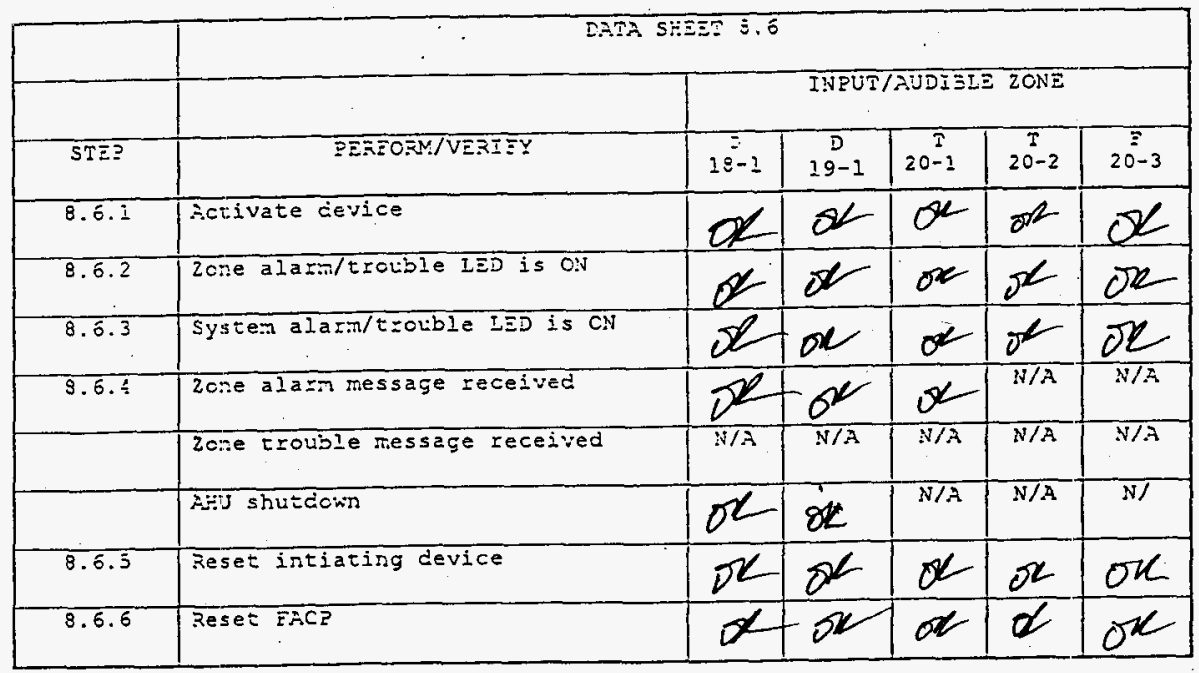

29 of 41 

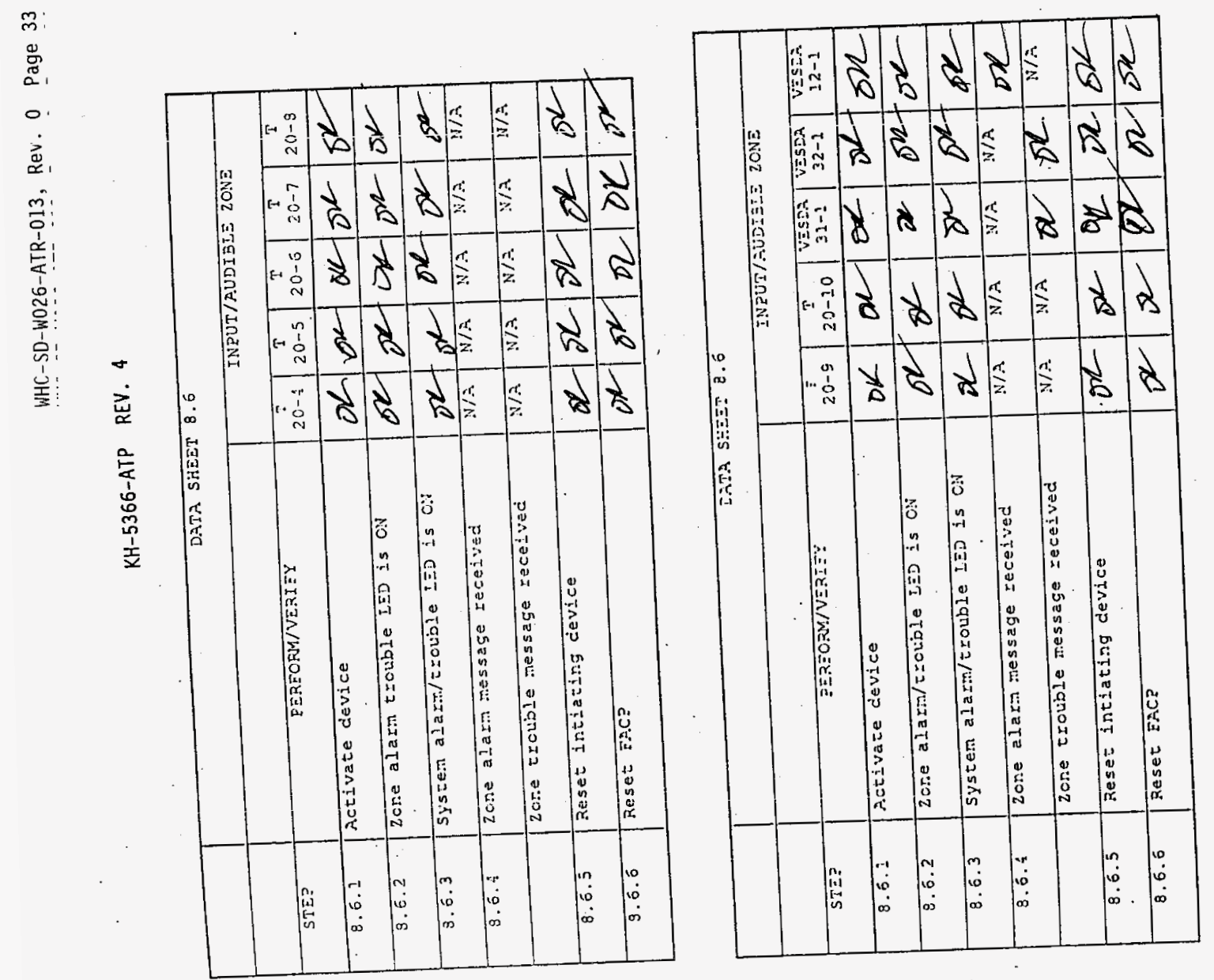


\section{KH-5366-ATP REV. 4}

$\operatorname{mog}^{\text {te }}$ N/A efeotion

EIGH/LOW PRESSURE SWITCB TEST (225pSi-20pSi)

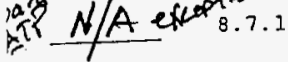

or

ix

$0 \% \quad 3.7 .4$

3.7 .5

or

196

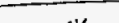

$+5$

of $\quad 3.7 .6$

\begin{tabular}{|c|}
\hline ok \\
\hline ok \\
\hline of \\
\hline
\end{tabular}

Test device H/I 33-1 for low pressure trip and record the pressure on data sheet 9.9 .7

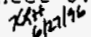

Verify EAC? zene trouble LED is on.

syster trouble IED is on.

Verify system trcuble message received.

Seset E:C?.

Test cievice :̈/I 33-1 for hich pressuze trip and record the presslize on disza sieet of 8.7

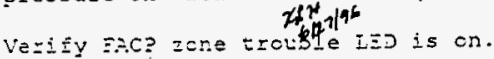

Verify systam trovile nessaga receivad.

Resez ZaC?.

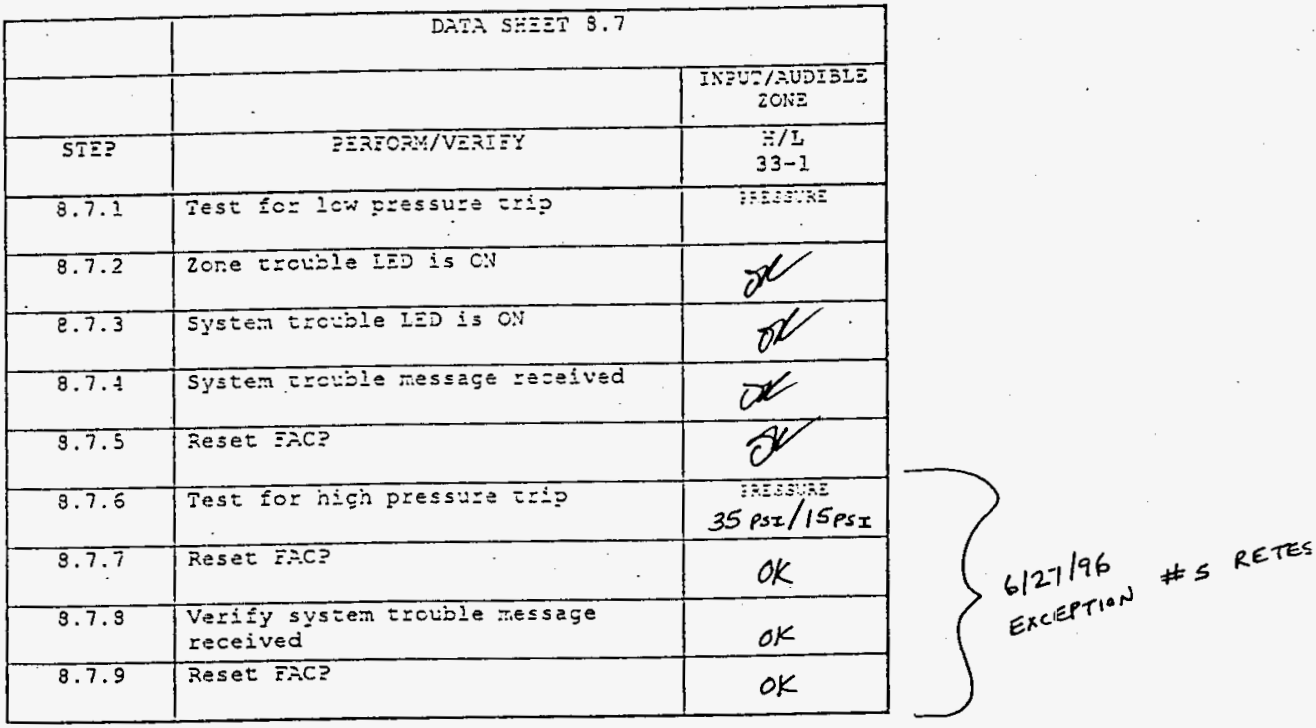


RE-5366-ATP REV. 3

8.8 PIV AND TAMPER SWITCH TESTING

8.8.1 Activate the supervisory device by turning the valve.

8.8.2 Record the number of turns required (less than 2 ).

8.8.3 Verify EACR zone trouble LED is on.

8.8.4 Verify FACP system trouble.

8.8.5 Verify REAR system trouble message received.

8.8.6 Reset supervisory device.

8.8.7 Reset FACP.

\begin{tabular}{|c|c|c|c|c|c|c|c|}
\hline & \multicolumn{6}{|c|}{ DATA SHEET 8.8} & \\
\hline & & & INPU' & AUDIB & ZONE & & \\
\hline STEP & PERFORM/VERIFY & $\begin{array}{c}\text { PIV } \\
34-1\end{array}$ & $\begin{array}{c}\mathrm{TS} \\
35-1\end{array}$ & $\begin{array}{c}\text { TS } \\
35-2\end{array}$ & $\begin{array}{c}\text { TS } \\
36-1\end{array}$ & $\begin{array}{c}\text { TS } \\
36-2\end{array}$ & \\
\hline 8.8 .1 & Trip device by turning value. & & & & & & $6 / 27 / 26$ \\
\hline 8.8 .2 & Record number of turns in 8.8 .1 & & & & & & xatf $6 / 27 / 26$ \\
\hline 8.8 .3 & EACP zone trouble LED ON & & & & & & $x$ a* $6 / 27 / 96$ \\
\hline 8.8 .4 & FACP system trouble LED ON & & & & & & xix $6 / 20 / 26$ \\
\hline 8.8 .5 & RFAR trouble message received & & & & & & $20 \% 6 / 27 / 76$ \\
\hline 8.8 .6 & Reset device & & & & & & $x / 4+6127 / 26$ \\
\hline 8.8 .7 & Reset FACP & & & & & & 2064t 4/22/76 \\
\hline
\end{tabular}




\section{KH-5366-ATP REV. 4}

\subsection{ORERATION OF DRY CEEMICAI SYSTEMS AND SEDTDOHNS}

Simulate alarm condition by shorting across FACP terminals \#2 and 45 , or :Il, as appropriate for each dry chemical system. Simulate the trouble condition by lifting one end of the EOI device.

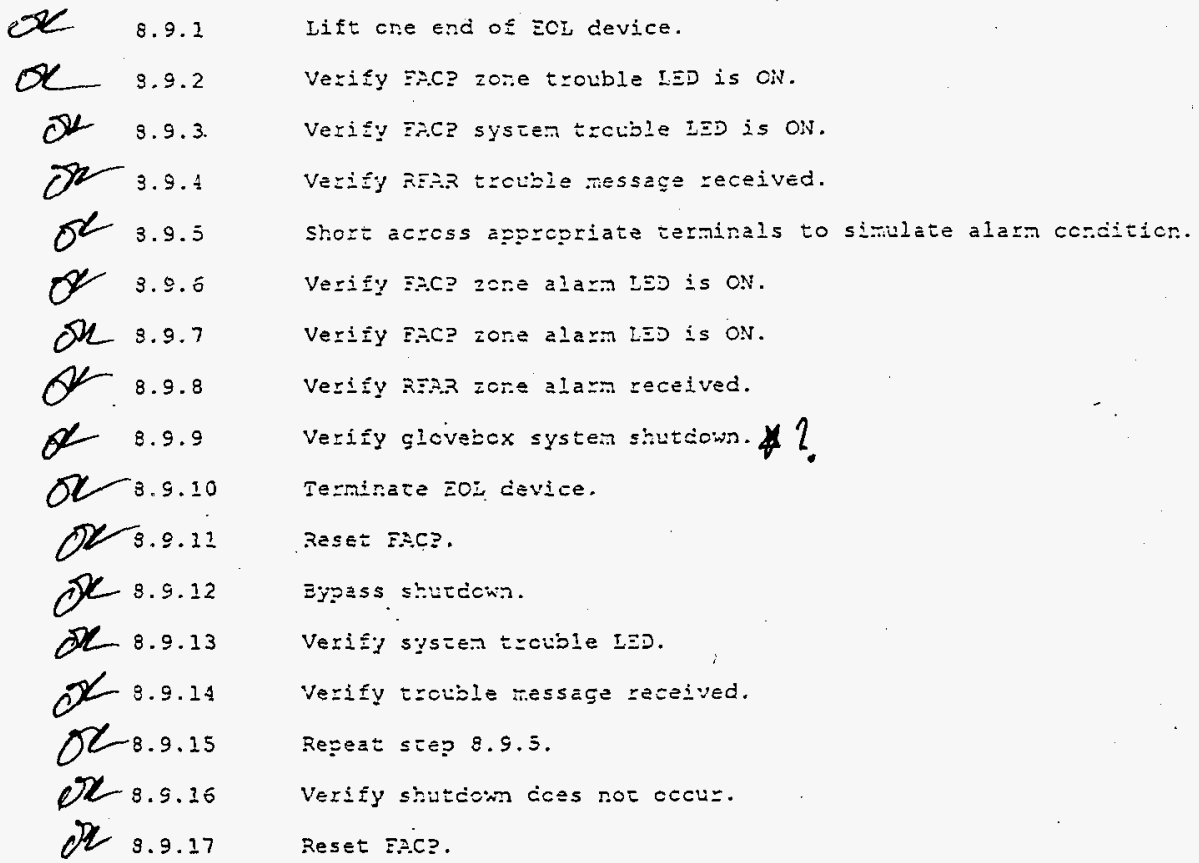


KH-5366-ATP REV. 4

\begin{tabular}{|c|c|c|c|c|c|c|c|}
\hline & \multicolumn{7}{|c|}{ DATA SHEET 8.9} \\
\hline & & & INEU & T/AUDI & SLE 2 & ONE & \\
\hline STE? & PEREORMTVERIEY & $\begin{array}{ll}0=-1 \\
i=-1\end{array}$ & 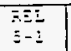 & (2) & 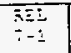 & $\begin{array}{ll}5=-1 \\
z-1\end{array}$ & - \\
\hline 3.5 .1 & Li:t cre end of $\overline{\mathrm{E} O I}$. & $d$ & or & $a$ & $\mathscr{O}$ & $0^{2}$ & $x$ \\
\hline 8.9 .2 & EAC? zone tratile LED ON & $\alpha$ & $\beta^{2}$ & a & or & or & $\infty$ \\
\hline 8.9 .3 & s:st=n Erouble IED CiN & or & $r^{2}$ & $y$ & $d$ & or & 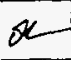 \\
\hline 8.9 .4 & $\begin{array}{l}\text { Aras :rcuble message } \\
\text { zeceived }\end{array}$ & of & on & $0^{2}$ & $\alpha$ & th & oe \\
\hline$\overline{3.5 .5}$ & 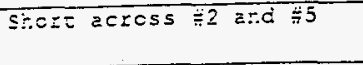 & $\phi^{\prime}$ & W/A & or & $\mathrm{N} / \mathrm{A}$ & si & $\overline{N / s}$ \\
\hline & stort across $\sharp 3$ and $\ddot{n} 11$ & $\because / \div$ & $\mathscr{F}$ & $N / A$ & $a$ & $i / \lambda$ & 0 \\
\hline $8 . \overline{9} .6$ & ZAC? zore ala:N IED ON & $\alpha$ & $\partial x$ & or & o & $x^{2}$ & sk \\
\hline 3.9 .7 & System alarm IED ON & $\theta$ & $\infty$ & or & $c$ & $x$ & Jw \\
\hline 8.0 .5 & $\begin{array}{l}\text { RExh alarm message } \\
\text { =eceived }\end{array}$ & $\bar{Z}$ & 2 & $d$ & of & $\mathbb{x}$ & st \\
\hline 3.9 .5 & Glovedox syste- shutcewh & $0 x$ & $\not 2$ & $\infty$ & or & \& & $\Delta 2$ \\
\hline 8.9 .10 & Tezinaze zoL cevice & qu & QL & or & $\theta$ & $d e$ & O4 \\
\hline 3.9 .11 & रeset: 2 Ac? & $\alpha$ & $\theta$ & $\mathscr{y}$ & $o^{2}$ & $\infty$ & B \\
\hline 8.9 .12 & Eyeass shutcionits & $\phi$ & $\mathbb{N}$ & Q & or & $D$ & or \\
\hline 8.9 .13 & Systam trcLble LED ON & $x$ & st & $D$ & $a$ & 5 & foc \\
\hline $8.9 .1 \frac{1}{4}$ & $\begin{array}{l}\text { Raza trouble nssace } \\
\text { recoived }\end{array}$ & on & ot & 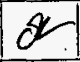 & OV & $\mathbb{Z}$ & $0^{2}$ \\
\hline 8.9 .15 & Repeat step 8.5 .5 & ox & 2 & 12 & or & $\pi$ & to \\
\hline 8.9 .16 & Shutdown does net occur & $x$ & Dr & $O L$ & Oh & $\mathbb{R}$ & ot \\
\hline $8 . \overline{9} . \overline{17}$ & Sesec $\bar{Z}$.CF & $a$ & $\alpha$ & ON & $d$ & $\alpha$ & 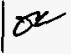 \\
\hline
\end{tabular}


WHC-SU-WO26-ATK-013, KeV. D Page 38

KH-5366-ATP REV. 4

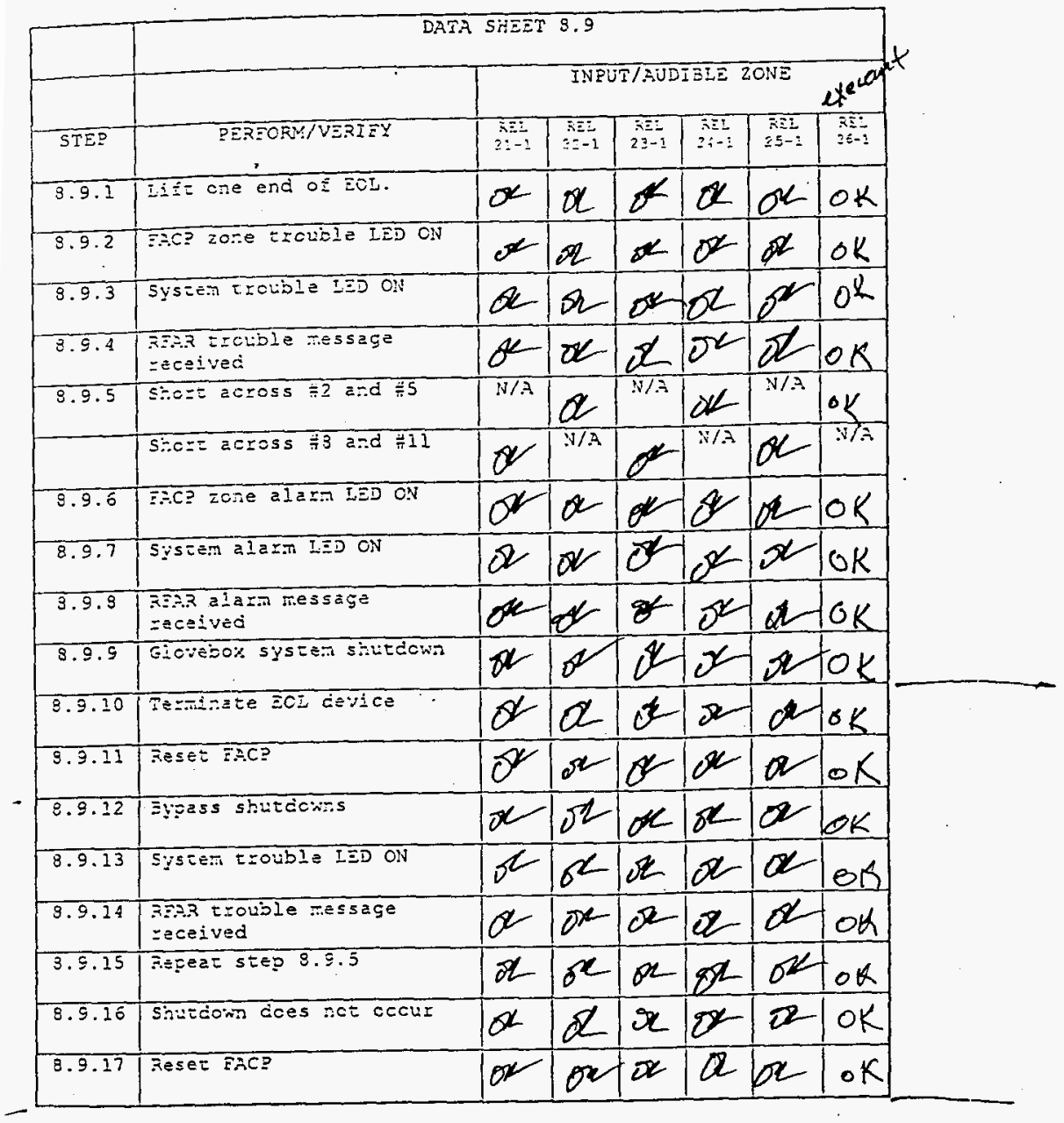

35 of 41 


\section{KH-5366-ATP REV. 4}

8.10 TESTING SUPERVISION OF AOXILIARY POWER SUPPLY

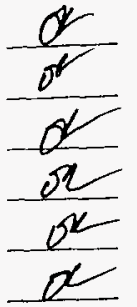

$8 \cdot 10.1$

8.10 .2

8.10 .3

8.10 .4

8.10 .5

8.10 .6

3.10 .7

8.10 .8

9.10 .9

8.10 .10

8.10 .11
Disconnect AC power from auxiliary power supply.

Vexify yellow fault LED is flashing.

Verify FAC? systen trolble LED is ON.

Verify systen trouble message receive.

Sestore AC power.

Very systen trolible testcred.

Reñve batzezy connecticn.

Sepeat steps $3.10 .2-9.10 .4$

Restore AC gowez.

Peconnect betzezy.

Verify systen nozmal. 


\section{KH-5366-ATP REV, 4}

8.11 TEST POHER SUPERVISION TO SFUTDOH'N RELAYS

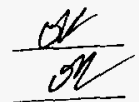

8.11 .1

Remove wite 4G-5 fron module 15, terminal 5 .

8.11 .2

Verify systen trouble IED is ON.

S.11..3

verify trouble message is received.

8.11. 4

Connect wile $i G-5$ to module $1 \vec{F}$, terminal 5.

8. 11.5

Verify systen rormal.

\subsection{TEST OF PIANT MANAGEANT SYSTEM (FNS)}

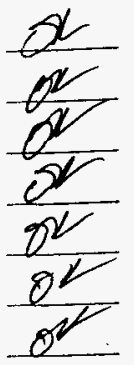

\$.12.1 Eypess bells ard strcbes.

8.12.2 Verify systen troule received by RMS.

8.12.3 Shor across terminals 2 a:d 5 , mociule $2 \mathrm{E}$.

8.12.4 Verify systen alarm receivec by PMS.

3.12.5 Reset EAC?.

8.12.6 Tutn bell byass swizch to nomal.

8.12 .7

VExiły systan cleaz of alarns and trouble, 


\subsection{BATTERY DRAW DOWN}

This test will verify battery capacity and system recharge capability as well as verify audibility of the fize alarm appliances.

or

$\gamma 2$

$\alpha 2$

529.13 .4

ON

8.13 .5

8.13 .6

9.13 .7

8. 13.8

8.13 .9

8.13 .10

8.13.11

9.13 .12

8.13 .13

8.13 .14

8.13 .15
Disconnect one battery lead and verify FAC? system toctile IED is C.N.

Connect ammeter in battezy/ganel circuit and verify Ej.c sysien cEoLible IED is OEE.

kecord bactery voltage.

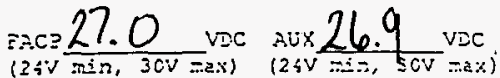

Record batzeyy curgent.

cyen the Fac? sypoly breaker to intartuge AC gcier. Secosd cies/time $6 \mathrm{rll}-47 \% 10110$

Verify AC po:ver crouble ircication.

secord battery voltage.

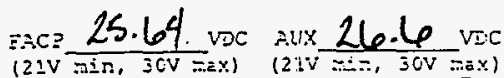

Fecord battery disciarg

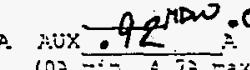

\section{Recectis}

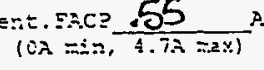
$\therefore$

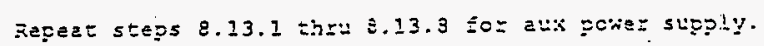

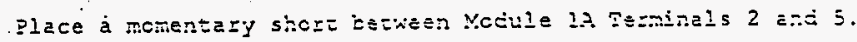

Verify that buildirg alaris sourd and Elash.

2lace a momentazy short betiest Kccule 1. Teminals 8 atc 11.

Vezify EAC? Zcnes 1 and 2 and RZXR zone alezan LEDs are CN.

Vezify three rcurds of fize alang ressages 2730 "Zoise I" ard 2730

"Zone 2" are received in sezwence by tre dispatcher.

Verify builcing alarms scusp for a minimum oz 5 minutes, anc perform steps 8.13 .16 and 3.13 .17 du=irg this period.

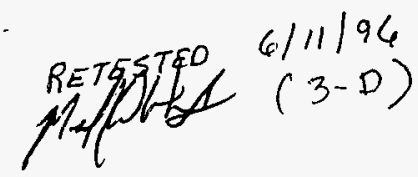




\section{KH-5366-ATP REV. 4}

\subsection{BATTERY DRAW DOHN}

This test will verify battery cepacity and system recharge capability as well as verify avibility of the fize alarm eopliances.

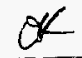

$8 n$

OK

OK

$O K$

8.13.5

8.13 .6

8.13 .7

8.13.8

8.13 .9

8.13 .10

8.13 .11

8.13 .12

8.13 .13

8.13 .14

8.13 .15

se
Disconect one tattery lead and verify EAC? system trotble LED is OiJ.

Connect amneter in battery/panel circuit and verify Fac? system ErCuble I.ED is OFE.

Fecord bettery voltage.

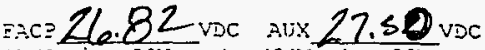

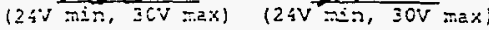

Secord batrery curzent.

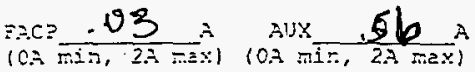

Coen the EAC? supply breaker to inzezzlot AC poier. Kecozd ciseltine $5 / 2 / x_{0} / 5 i 15$

Verify IC poiser rrouble ircicarich.

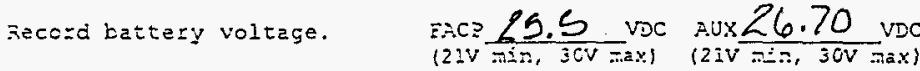

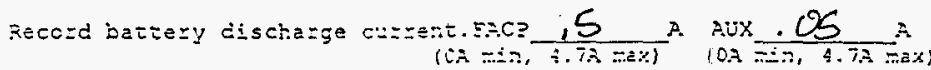

kepeat steps 8.33 .1 thzu 3.13 .3 for aux gcrez sueply.

2lace a monentery short dezieen Yocule ia Tezminals 2 and 5.

Verify that building alams sound and Elash.

2lace a nomentazy short befween Vocule lA Teringls 8 and 11.

Verify FaC? Zones 1 and 2 and REPR zone alazn LEDs are CN.

Verify three rcuzds of fize alazn messages 2730 "205e 1" and 2730

"Zore $2 "$ are received in secuence by tre cispatcher.
Verify building alarns scuid for a minimun of 5 minutes, ard gertorn steps 8.13 .16 and 8.13 .17 cuzing this perjod. 
Corcuct a walking survey of the entire area serviced ty aucible alarms cperated by the FAC? of this AT2. Verify all aucible devices have a sound 10cb above background at the time of test. Record on data sheets 8.13.16. Document nonccmoliances as exception to the ATP.

8.13 .17

8.13 .18

$3 .: 3.19$

8.13 .20

9.13 .21

3.13 .22

9.13 .23

$\varepsilon .13 .24$

8.13 .25

Verify all stzcbes. Record on data sheet 8.13.17.

Record battery roltage.

IAc? 24.45 vDC (2.05V/ee11)

Record discharge current.

silence alern gcrgs.

Close ard secure I:CC? st:zly bzeaker.

Recosd ba: $=$

EC? Is 18.53

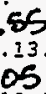
A... 10.05 diegce curreas

Ex.C?

(0.) min, 23 न तex A.: Is 18.13 .5

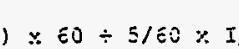

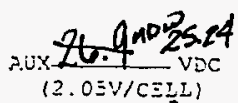

A vid 10 Nong (OA min, 4.7. max) (CA min, 4.7.A max)

of

\subsection{6 \\ $.191=3318_{\text {fur }}$ sours.

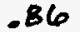 \\ $=3.072: 12: 0:=s$.}

RETESTEP

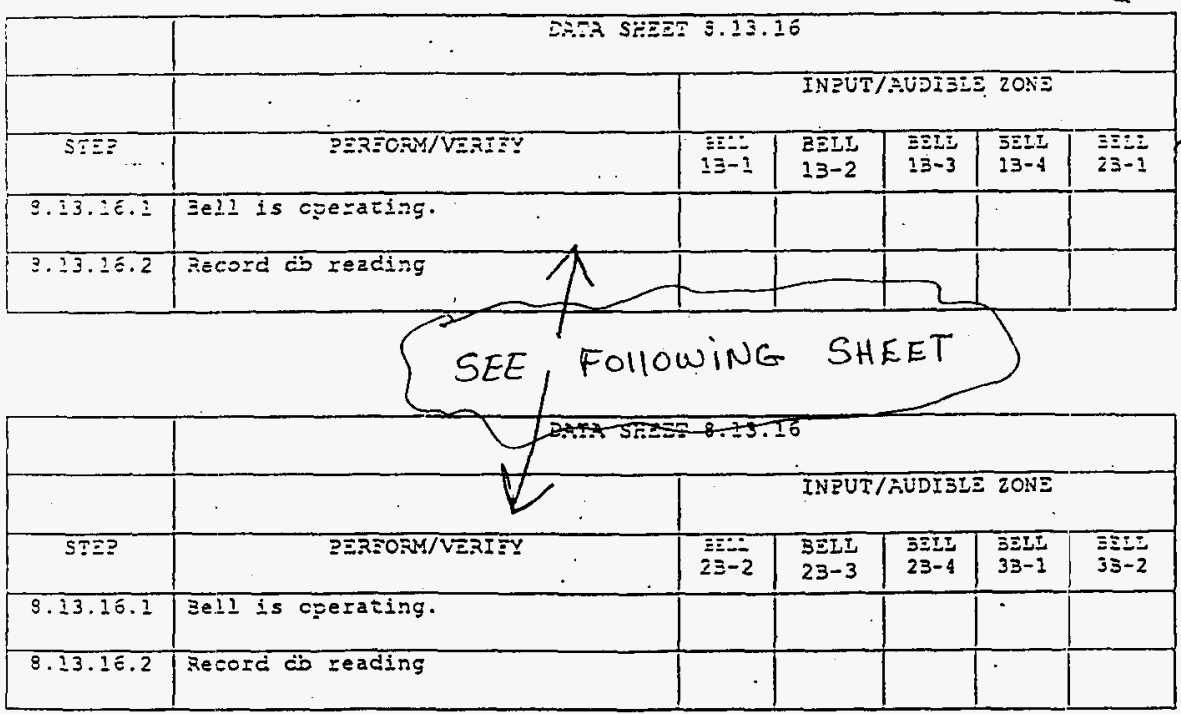


8.13.16 Conduct a walking survey of the entire area serviced by audible alarms operated by the FACP of this ATP. Verify all audible devices have a sound lodb above background at the time of test. Record on ciata sheets 8.13.16. Document noncompliances as exception to the AIP.

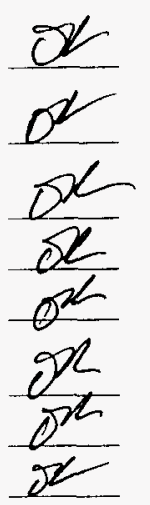

8.13.17 Verify all strobes. Record on data sheet 8.13.17.

8.13.18 Record battery voltage.

FACP $\frac{24.6}{(2.05 \mathrm{~V} / \mathrm{cel} 1)} \mathrm{VDC}$

AUX 24,8 \{2.05V/CELL\}

8.13.19 Record discharge current. EACP Z.5 A AUX 28

8.13.20 Silence alarm gongs.

(OA min, 4.7A $\max$ ) (OA $\min , 4.7 \mathrm{~A} \max$ )

8.13.21 :Close and secure FACP supply breaker.

8.13.22 Record battery charge current EACP 1.59 A AUX-3.4

8.13.23 Reset FACP.

8.13 .24

FACP IS $(8.13 .8) \times 60+5 / 60 \times I_{\mathrm{n}}(8.13 .19)=302_{\text {AMP }}$ hours.

8.13 .25

Aux Is $(8.13 .8) \times 60+5 / 60 \times I_{3}(8.13 .19)=3.2$ AMP hours.

\begin{tabular}{|c|c|c|c|c|c|c|}
\hline & \multicolumn{6}{|c|}{ DATA SHEET 8.13 .16} \\
\hline & & \multicolumn{5}{|c|}{ INPUT/AUDIBLE ZONE } \\
\hline STE? & PERFORM/VERIFY & $\begin{array}{c}5 E \\
1 B-1\end{array}$ & $\begin{array}{l}\text { BELI } \\
13-2\end{array}$ & $\begin{array}{l}\text { BELI } \\
1 \mathrm{~B}-3\end{array}$ & $\begin{array}{l}\text { BELL } \\
1 B-4\end{array}$ & $\begin{array}{l}\text { BELI } \\
2 B-1\end{array}$ \\
\hline 8.13 .16 .1 & Bell is operating. & & & 20 & & \\
\hline 8.13 .16 .2 & Record db reading & & & $\begin{array}{l}\text { 85/65 } \\
\mathrm{RH} / 34\end{array}$ & $\begin{array}{l}22 / 72 \\
\text { endile }\end{array}$ & $78 / 81$ \\
\hline
\end{tabular}

\begin{tabular}{|c|c|c|c|c|c|c|}
\hline & \multicolumn{6}{|c|}{ DATA SHEET 8.13 .16} \\
\hline & & & INPU & AUDIB & ZONE & \\
\hline STEP & PERFORM/VERIFY & $\begin{array}{l}E E:-i \\
2 B \sim 2\end{array}$ & $\begin{array}{l}\text { BELL } \\
2 B-3\end{array}$ & $\begin{array}{l}\text { BELL } \\
2 B-4\end{array}$ & $\begin{array}{l}\text { BELL } \\
3 B-1\end{array}$ & $\begin{array}{l}\text { BELL } \\
3 B-2\end{array}$ \\
\hline 8.13 .16 .1 & Bell is operating. & & & & & \\
\hline 8.13 .16 .2 & Record db reading & 2874 & $70 / 24$ & $6 \times 79$ & 64780 & 6780 \\
\hline
\end{tabular}


KH-5366-ATP REV. \&

8.13.16 Conduct a walking survey of the entire area sezviced by audible alarms operated by the FACP of this ATP. Verify alli audible devices have a sound $10 \mathrm{db}$ above background at the time of test. Record on data sheets 8.13.16. Document noncompliances as exception to the ATP.

8.13.17 Verify all strobes. Record on data sheet 8.13 .17 .

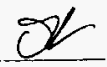

8.13.18 Record battery voltage.

EAC? $24 . \leqslant$ VDC

AUX 24.8

$$
\text { (2.05V/cel1) }
$$

(2.05V/CEIL)

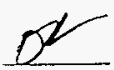

8.13 .19

Record discharge current.

EACP 2.5 AUX 28

(OA min, 4.7A max) (OA min, 4.7A max)

8.13.20 Silence alarm gongs.

8.13.21 :Close and secure EACP supply breaker.

8.13.22 Record battery charge current EACP 1.59 A AUX-3.4 (OA $\operatorname{min,} 2 A \max )$ (OA MIN, 2A max)

8.13.23 Reset FACP.

8.13.24 FACP $I_{3}(8.13 .8) \times 60+5 / 60 \times I_{n}(8.13 .19)=302_{\text {AMP hours. }}$

8.13.25 Aux $I_{3}(8.13 .8) \times 60 \div 5 / 60 \times I_{\lambda}(8.13 .19)=3.2$ AMP hours.

\begin{tabular}{|c|c|c|c|c|c|c|}
\hline & \multicolumn{6}{|c|}{ DATA SHEET 8.73 .16} \\
\hline & & \multicolumn{5}{|c|}{ INPUT/AUDIBIE ZONE } \\
\hline STEP & PEREORM/VERIFY & $\begin{array}{l}E \equiv E \\
I B-1\end{array}$ & $\begin{array}{l}\text { BELI } \\
1 B-2\end{array}$ & $\begin{array}{l}\text { BELL } \\
1 B-3\end{array}$ & $\begin{array}{l}\text { BELI } \\
1 B-4\end{array}$ & $\begin{array}{l}\text { EELI } \\
2 B-1\end{array}$ \\
\hline 2.13 .16 .1 & Bell is opezating. & & & 2 & & $\infty$ \\
\hline 3.13 .16 .2 & Record db reading & & & $\begin{array}{l}3 / 6 t \\
R M / 34\end{array}$ & $\begin{array}{l}22 / 72 \\
\text { crille }\end{array}$ & $7 \% / 81$ \\
\hline
\end{tabular}

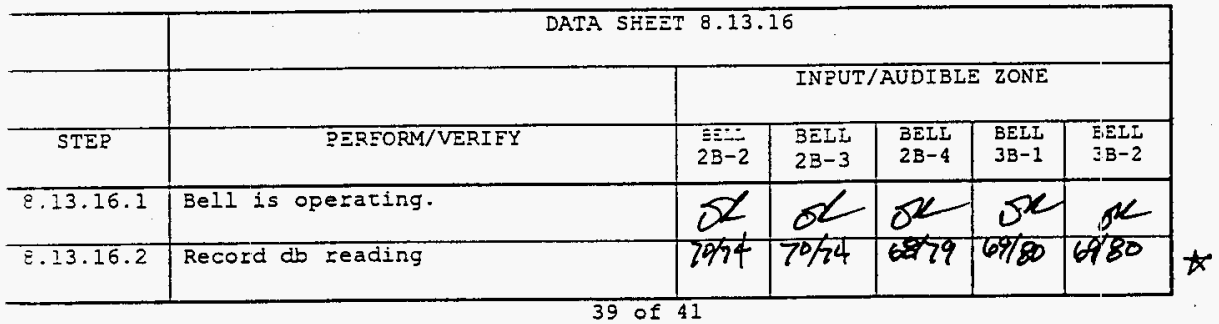

* SEE atTacheo industrial hycFists REPRT. EXeEPTIN \#6. 
KH-5356-ATP REV. 4

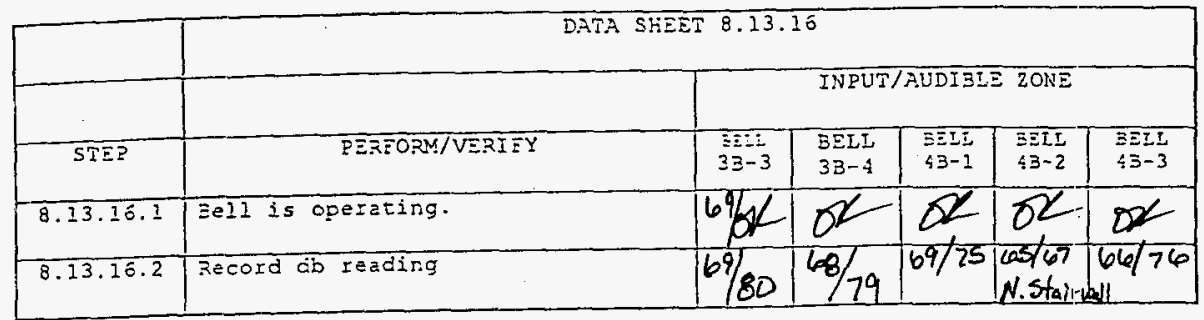

\begin{tabular}{|c|c|c|c|c|c|c|}
\hline & \multicolumn{6}{|c|}{ IAT S:IEET $\$ .13 .17$} \\
\hline & & & INPUT & SUDE三E三 & ZCNE & \\
\hline $5 T=?$ & EERECRM/VERIZY & $\begin{array}{l}3=\mathrm{s}: 5 \pm \\
15-1\end{array}$ & $\begin{array}{l}3-5: 5 \\
15-2\end{array}$ & $\begin{array}{c}2+2+\infty \\
1 S-3\end{array}$ & $\begin{array}{l}\text { into: } \\
\text { is }-4\end{array}$ & $\begin{array}{c}5: 5 \equiv \\
15-5\end{array}$ \\
\hline 8.13 .27 & sircice is creratirg. & & & & & \\
\hline
\end{tabular}

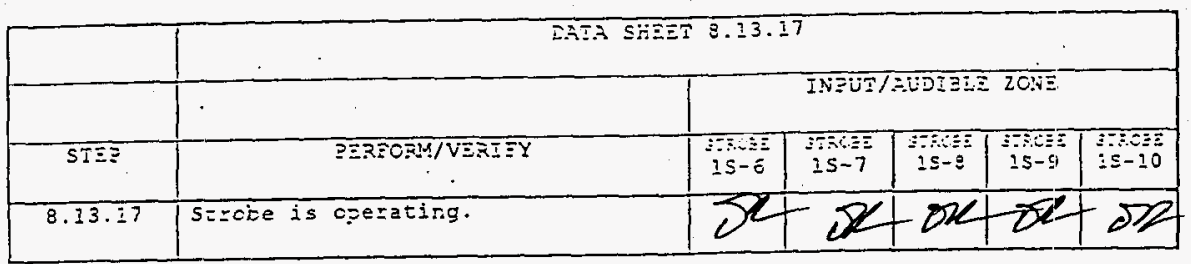

\begin{tabular}{|c|c|c|c|c|c|c|}
\hline & \multicolumn{6}{|c|}{ DETA SHEII 8.13 .17} \\
\hline & & & INDUT/ & UDะラİ & $20 N:$ & \\
\hline STE? & ZEREORM/VERIEY & $\begin{array}{l}3205 \overline{2} \\
15-11\end{array}$ & $\begin{array}{c}5 A 0 E \equiv \\
25-1\end{array}$ & $\begin{array}{c}3: S \equiv z \\
6 \$-2\end{array}$ & $\begin{array}{c}3-205 \\
25-3\end{array}$ & $\begin{array}{c}325 \\
25-4\end{array}$ \\
\hline 8.13 .17 & stzcice is cperating. & & & & & \\
\hline
\end{tabular}

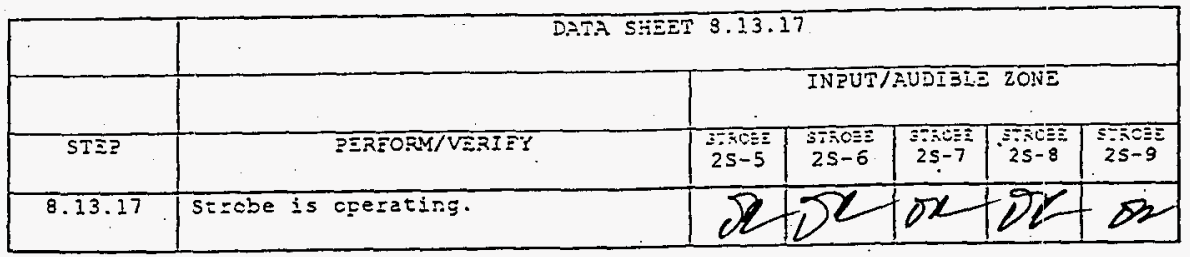


XH-5366-ATP REV. 4
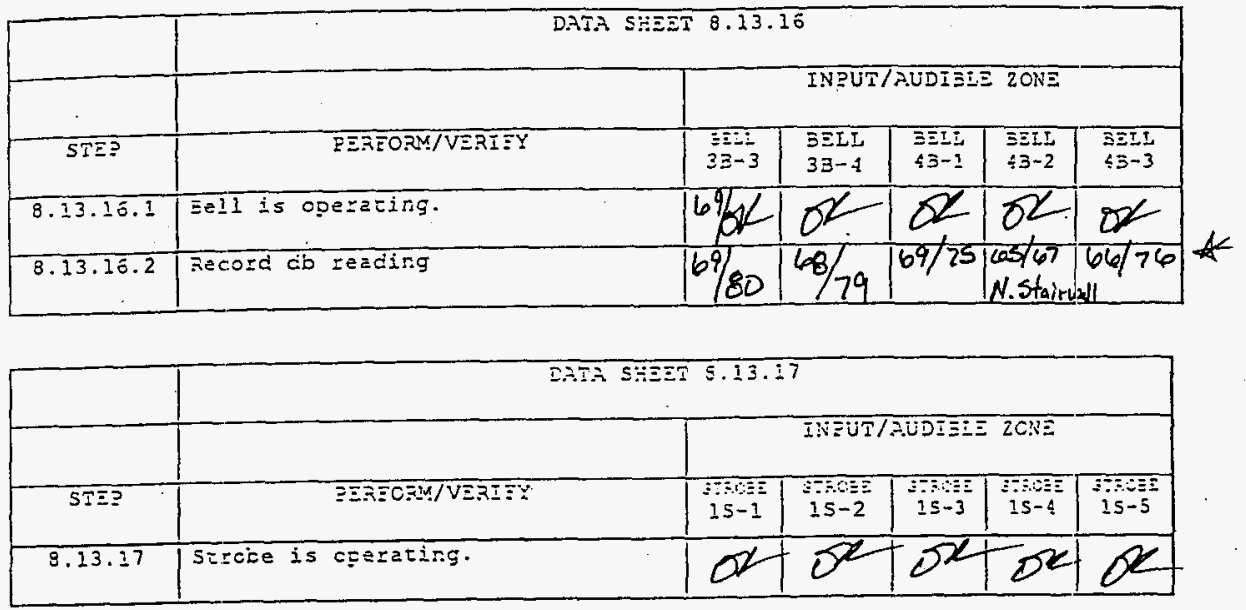

\begin{tabular}{|c|c|c|c|c|c|c|}
\hline & \multicolumn{6}{|c|}{ EATA SFEE? 8.13 .17} \\
\hline & & & 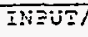 & 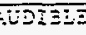 & $20 \times 5$ & \\
\hline STEE & IEREOEU!VERIEY & 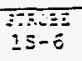 & $\begin{array}{c}\text { SREE } \\
\text { is }-7\end{array}$ & $\begin{array}{c}25-2 \\
15-2\end{array}$ & $\begin{array}{l}375=2 \\
15-9\end{array}$ & $\begin{array}{l}5: 5 \\
\vdots 5-10\end{array}$ \\
\hline 8.13 .17 & strciee is cperatirg. & & & & & \\
\hline
\end{tabular}

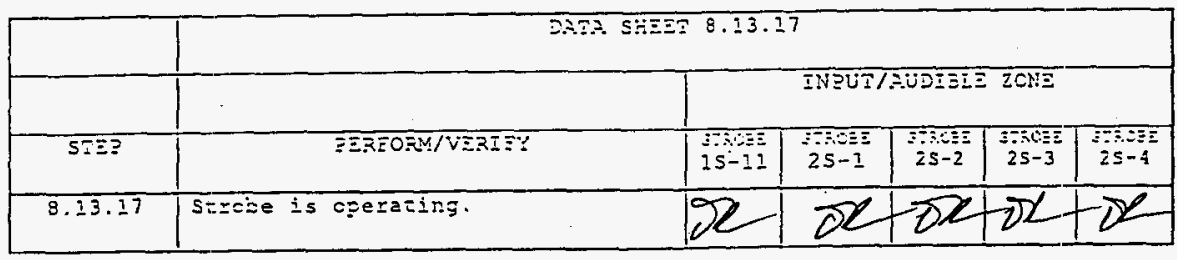

\begin{tabular}{|c|c|c|c|c|c|c|}
\hline & \multicolumn{6}{|c|}{ D.XA SHEET 3.13 .17} \\
\hline & & & INEUT & UDISE & $201 \% \mathrm{E}$ & \\
\hline $5 \bar{T} \equiv ?$ & 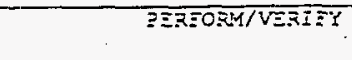 & $\begin{array}{c}2705 \\
2 s-5\end{array}$ & $\begin{array}{c}5 \pi C E E \\
25-6\end{array}$ & $\begin{array}{c}250 \equiv \\
2 s-7\end{array}$ & $\begin{array}{c}S=R O E: \\
2 s-8\end{array}$ & $\begin{array}{c}5 \text { SरCEE } \\
25-9\end{array}$ \\
\hline 8.13 .17 & $s=$ cloe is cperatirg. & & & & & \\
\hline
\end{tabular}


WHC-SD-W026-ATR-013, Rev. 0 Page 48 KH-5366-ATP REV. 4

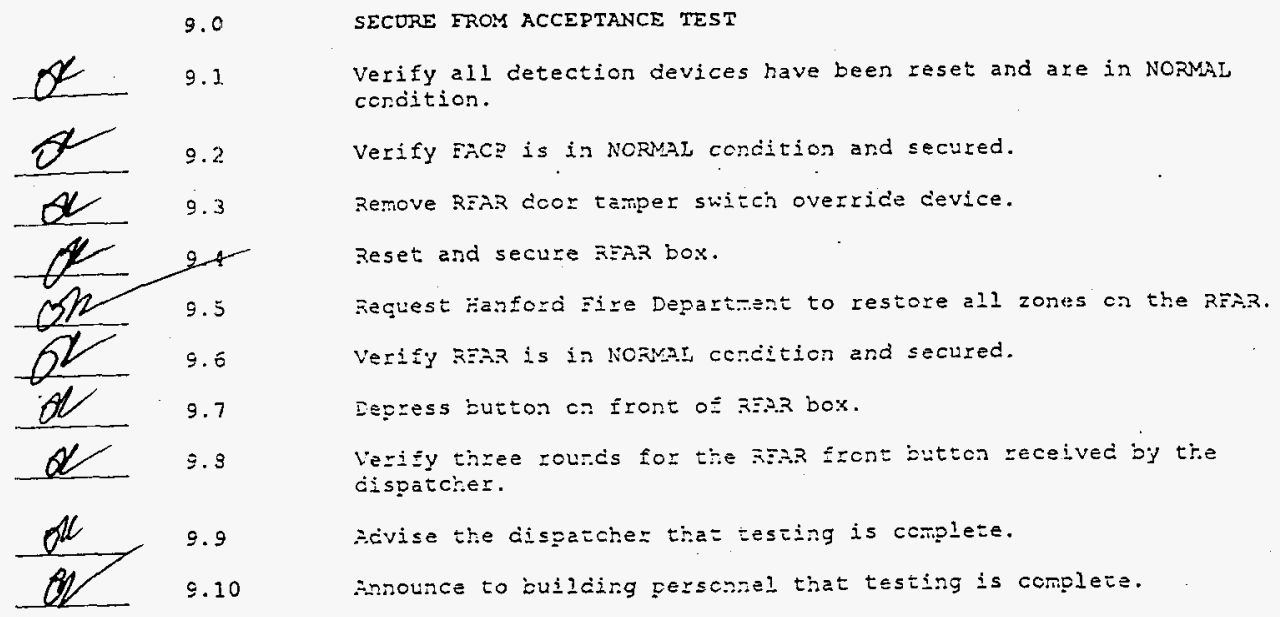

END OE TEST

41 of 41 
KH-5366-ATP REV. 4

ADPENDIX A

INSTAILER CHECRIIST

A-1 


\section{KH-5366-ATP REV. 4}

\section{APPENDIX A}

\section{A. PURPOSE}

This apcendix is provided as a chectlist of activities and rezifications to be cerformad by the fire alarn systen installer to ensure proper installation and cperation. Responsibilicy for complecion of the Eollcwing items are the respcrsiblility of the installer, botin to meet ND2A 72 H installacion testing reguirements and to insure that tre IIP proceecis in en efficient manner.

\section{B. INSTALLER}

1.0 Erior to cerminaticn concuctors that have been laic or pullec outsice of

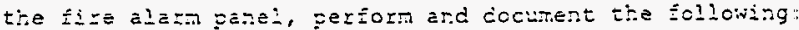

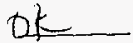

1.1 Verify that all concuctors cher than thcse intentionaly and

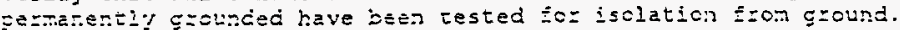

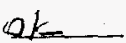

1.2 Vezify $t h \equiv=\equiv 11$ conductors have teen tested for ccacuctoz-toconductor isclaticn.

1.3 Verity tine exterral circuiz loop resistance neasurenents are within the following limits:

a. Waxima $3 \tilde{c}$ chas for initiating device ingut circuits.

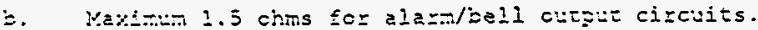

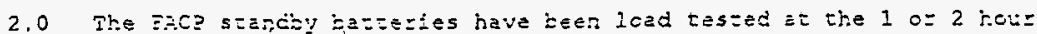

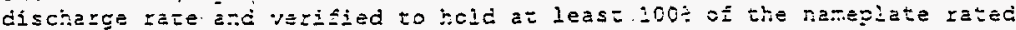
capacizy. The dazzeries have been recharged and rave been cu a charger tor ac least 43 icoss in the past week.
a. Eastery exclosure datzeries

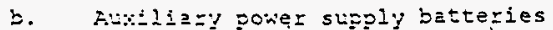

3.0 Complete arca sumiz alarm systen Cerzificate of compliance in accorciance with $: \bar{v}=2 \mathrm{~A} 72$. 


\section{KH-5366-ATP REV. 4}

\section{INSTALIER AND INSPECTOR}

After completing tie terminations of the system and conductors in accorciance with the installation drawings, perform and verify the following:

of 2. 2. Verizy that the systen is installed and wired in accordance with the Oł

2. Inspect each initiating device and alarn cutput cevice and verify that the corcletors are p=ozerly wired for supexvision.

Dt_

QLE

$\underline{0 k}$

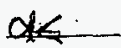

$2 E$

ok
3. Verify tiat each recuized erd-of-line cevice is in place.

4. Witi jattery installed and normel power suppled, verify that.the Fac? is in a NCELI supervisozy conditich as cefined by lie manufacturer's marial.

5. Ey lizing leacis cr enci-cf-line cevices, verify that each sugervised zone Eroduces proper rrcuble incication.

6. Verify that each alaza/supervisozy initiating device prodicss p=coer alarn corcitions it:en activatec.

7. Verify tiat alazk cells/chimes/stzcies operate properly.

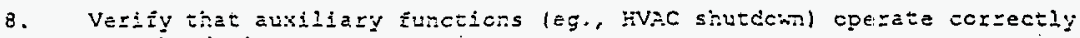
pez the design. 
WHC-SD-W026-ATR-013, Rev. 0 Page 52

KH-5366-ATP REV. 4

APPENDIX 3

EXCEYTION SEEETS

$3-1$ 


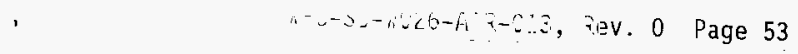

$$
\begin{gathered}
\text { KH-5366-ATP REV. } 4 \\
\text { EXCEPTION SEEII }
\end{gathered}
$$

EXCEPTION NUMEZR:

DATE: $5 / 3 / 96$

EXCEITION: DRAWINE CHANGES SEE ATTAC HED.

ELAZY:D ACITON: AS BUILT

P.CIICN T:XZEN:

DA: : $7 /$ /iil 96

THE TRACKING OF THE AS-BUILT DRAWINGS IS BEING ACCOMFLISHED BY THE ITEM \#6 ON. THE OFFICIAL PROJECT RUNCHLIST, "WRAP Module I Spec Section 16720 Fire Alarm and Smoke Detection," DATED 5/13/96.

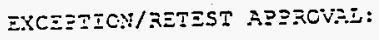

jetest agp:oved anc accspted
E๐cežich

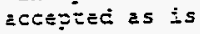

(jiesd $\dot{c}=a i$ Is)
C-ise

(Nesd citails)

ㄱeะaミ1s:

AS-BUILTS ARE STILL NEEDED. THE PUNCHLIST WILL ENSURE THAT AS-BUILTS ARE PROVIDED.

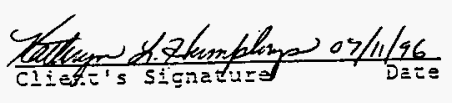

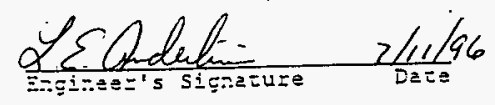

Brece ofanz $7-11-96$

$3-2$ 
FACP Exceptions:

1. Drawing Changes:

a. Designate wiring \# \& breaker \# for RFAR and FACP

RFAR X11733, 23, PP-11-102 A, electric room FACP X11734, 39, PP-11-102 A, electric room

b. Bells, strobes, pull stations located incorrectly $1 \mathrm{~S}-5,1 \mathrm{~B}-2,2 \mathrm{~S}-6,2 \mathrm{~B}-3$ (relocate on drawing)

c. EOL located incorrectly (relocate on drawing) zone 10,14 and 20

$E O I=10-40,14-7,20-9$

d. Zone 36 devices do not exist (remove from drawing)

e. Zones 27 and 28 are "flow switches" for "main alarm check valve" and "hose rack"

f. Both Process Area AHU's and Computer Control Rocm AHU's shutdown when required

g. Only zone 11 needs to shutdown equipment inside the GB detecting fire

h. There are 2 heat detectors in the shipping/receiving area which axe not shown on the drawing

i. FACP $A$ and FACP $B$ need to be reversed on plan 


$$
\text { WHC-SD-W026-ATR-013, Rev. } 0 \text { Page } 55
$$

KH-5366-ATP REV. 4

EXCEPTION SEEET

EXCEPICN NLMEER: _______ DATE: $5 / 3 / 96$ E:CEEIIO: _.STROBES CAN NOT BE BYPASSED ( PAGE 77

PTDNNED ACIICN:

SubmiT RFI

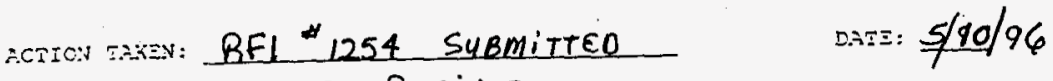
ANSWER RECineD

EXCEETICN/RETEST APPRCVEI:

Ferest agproved asci accepted $\exists x \in=\leftarrow c \mathrm{c}$

acc\%ptec as is

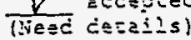

Coider

Theac cisizis\}

F⿻tละ1s:

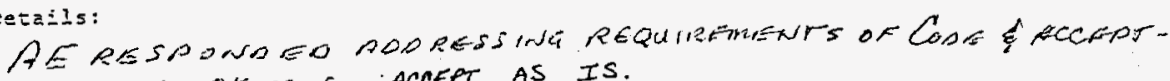
DBLVTH OKNS IS. ACCEPT AS IS.

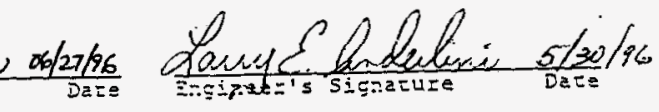

Marl 1 Bran $7-2-46$

ICF-KU AT

$3-2$ 


\section{KH-5366-ATP REV. 4 \\ EXCEYTION SEEET}

EXCEZTICN NLMEER:

3

DATE:

EXEETEN: DID NOT TEST ZONES $27+28$ RELABLE*27 "MAIN ALARM CHECK UAIVE FIOW SWITCH"

?TMNED :CTICN: TEST ON Follow uP ATP.

ACTIC: IEN: TESTED ZONES 27 ANO 28 5AIE: $5 / 20 / 96$ INSTALLED NEW LABLE

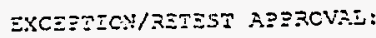

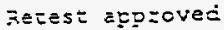
a.sci accepted

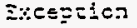
accezied as is

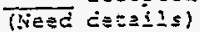
$c=$ ser

(Xiecc ceta11s)

Dะこวシปร :

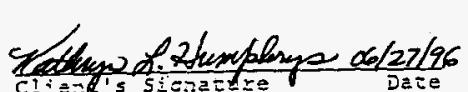

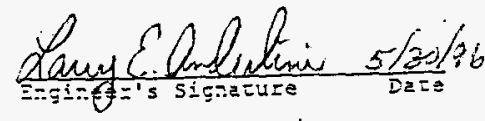

Mad' B AN

$2-1-96$

ICF.KH.AI 


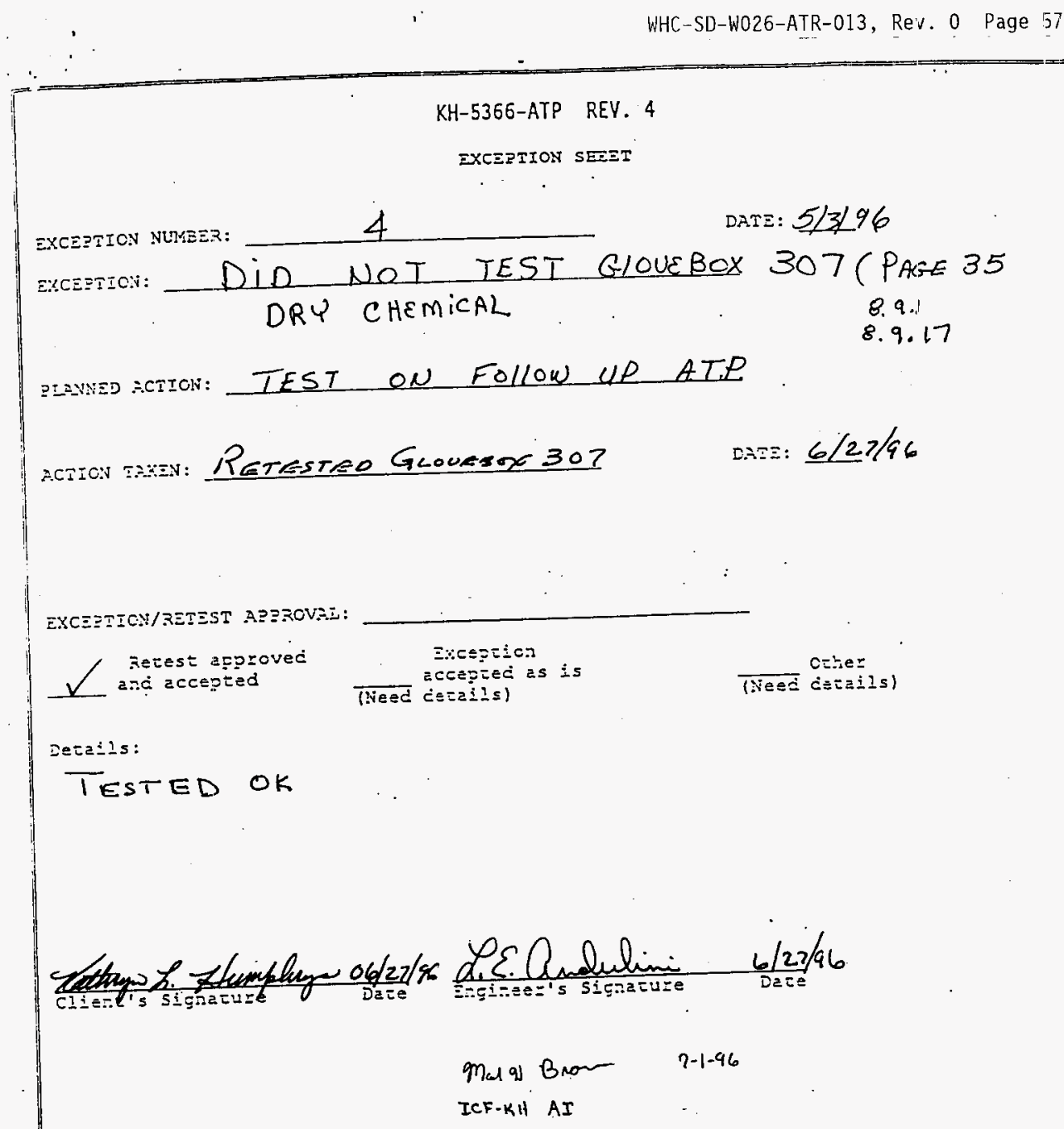




$$
\text { WHC-SU-W026-AIR-013, Kev. O Page } 58
$$

$$
\text { KH-5366-ATP REV. } 4
$$

EXCEPTION SHEET

EXCEZTICN NUMEER:

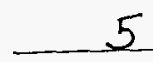

DATE: $5 / 3 / 96$

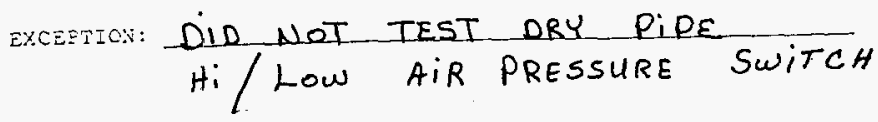

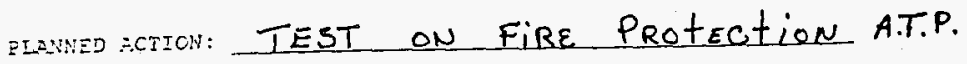

ACTION TXKEN: TESTED ON FiRE PROTECTION DATE: 5/20/96 A.T.P. PASSED REQUIRMENTS

EXCEZTICN/RETEST APPROVE:

netest acproved anci accepted

$\mathcal{L}$

Details:
Excspticn accepted as is

(Need eisails)
Ceter (Neec cerails)

Clthmughtumpling 06/27/96

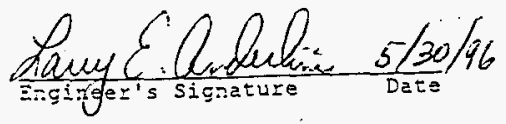

$\operatorname{Mod} B$ M

$9-196$

ICF-KH AI

$3-2$ 
KH-5366-ATP REV. 4

EXCEZTION SEEEI

EXCEPTTCN NLMEER:

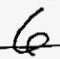

DAT $: 5 / 3 / 96$

EXCEZTC: NEED MORE BELLS AND STROBES

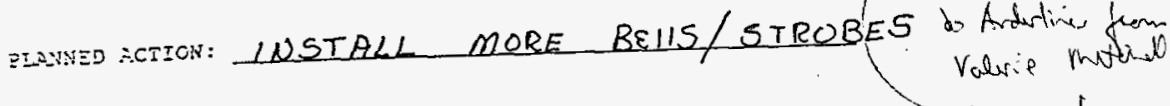

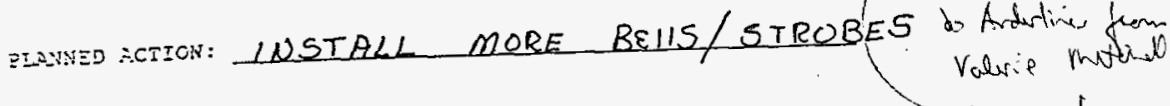
EL-INED ACTION: WSTALL MORE BEIIS/STROBES do Ardotine from sound bue the mond ACITC: TKEN: ADDED MORE BELLS AND STRO valus thatent

EXCEZTICN/RETEST A?ZROVEI:

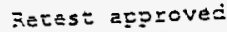
axci accepted

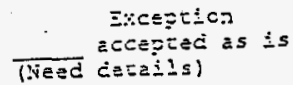

Crter

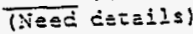

Deteizs:

Aoditional Srmoge chacelpe

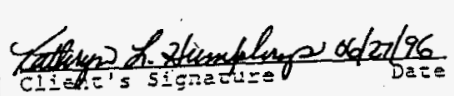

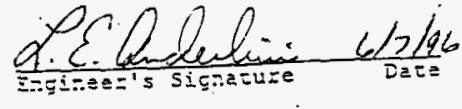

Mal of Brom

$7-1-96$

ICF-KH AI 


$$
\text { WHC-SD-W026-ATR-013, Rev. } 0 \text { Page } 60
$$$$
\text { KH-5366-ATP REV. } 4
$$$$
\text { EXCEYTION SEEZT }
$$

EXCEZTION NUMEER:

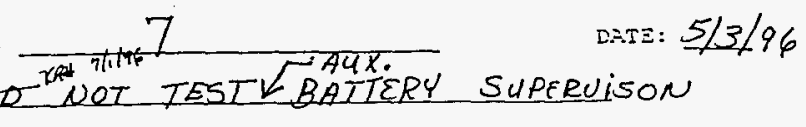

ELZWNED :CIICW: ACCEPT AS IS

ACIICN I:XEN: ACCEPT AS IS

DAIE: $7 / 1 / 96$

EXCEETICN/RETEST APJROVE:

secest approvec anc accopted

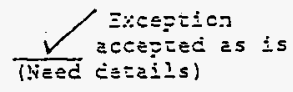
Citer (Nived esins) (Aives cisidis)

Detains:

THERE IS NO REOUIREMENT FOR THE AUX. BATTERY POWER TS BIE SUPERYISED IN THE CONSTRUCTION SPECIFICATIONS, NFPA 72, NOR NFPA 1221 .

THE CHANGE TO THIS ATP WAS REVIEWED/AUTHORIZES ON SUGMITTAL $15 T I . E$.

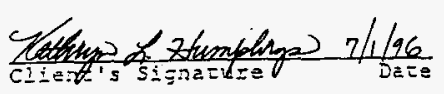

$$
\begin{aligned}
& \text { Q.E. Anslalimi } 7 / 2 / a 6 \\
& \text { Mald Bnow 2-1-96 } \\
& \text { TCF-Kit AI }
\end{aligned}
$$

$3-2$ 


\section{KH-5366-ATP REV. 4}

EXCEPTION SEZT

EXCESTICN NLMEER:

8

DAT $=5 / 3 / 96$

EXCEE?IC:H:

ZONE

36

NOI

TESTED

ETZNDED ECTICN: EXCEPT AS is (EUture ZONE)

F.CIIC: TAREN:

DลIอ:

EXCEETTCN/RETEST APPROVM:

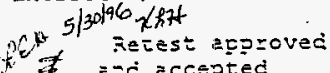

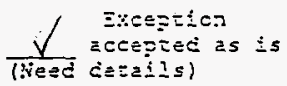
Csker

(Neec catails)

Detzis: As PMNEL Fxists THIS IS ASPARE ZONE

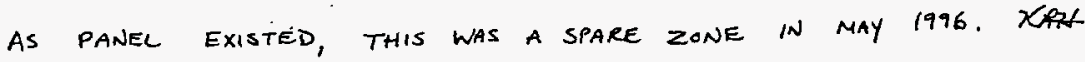

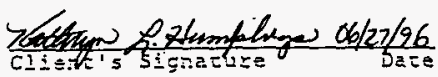

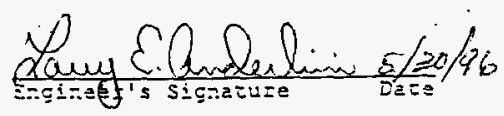

Mola BNom 7-1.96

ICF-KH AI 
$-\cdots$

PROCEDURE

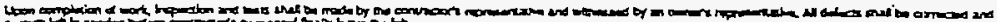

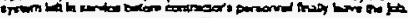

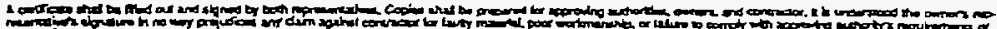

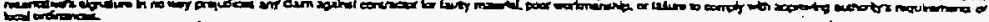

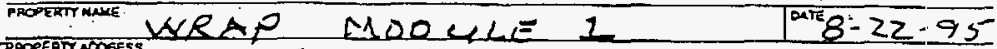

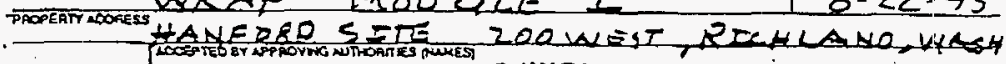

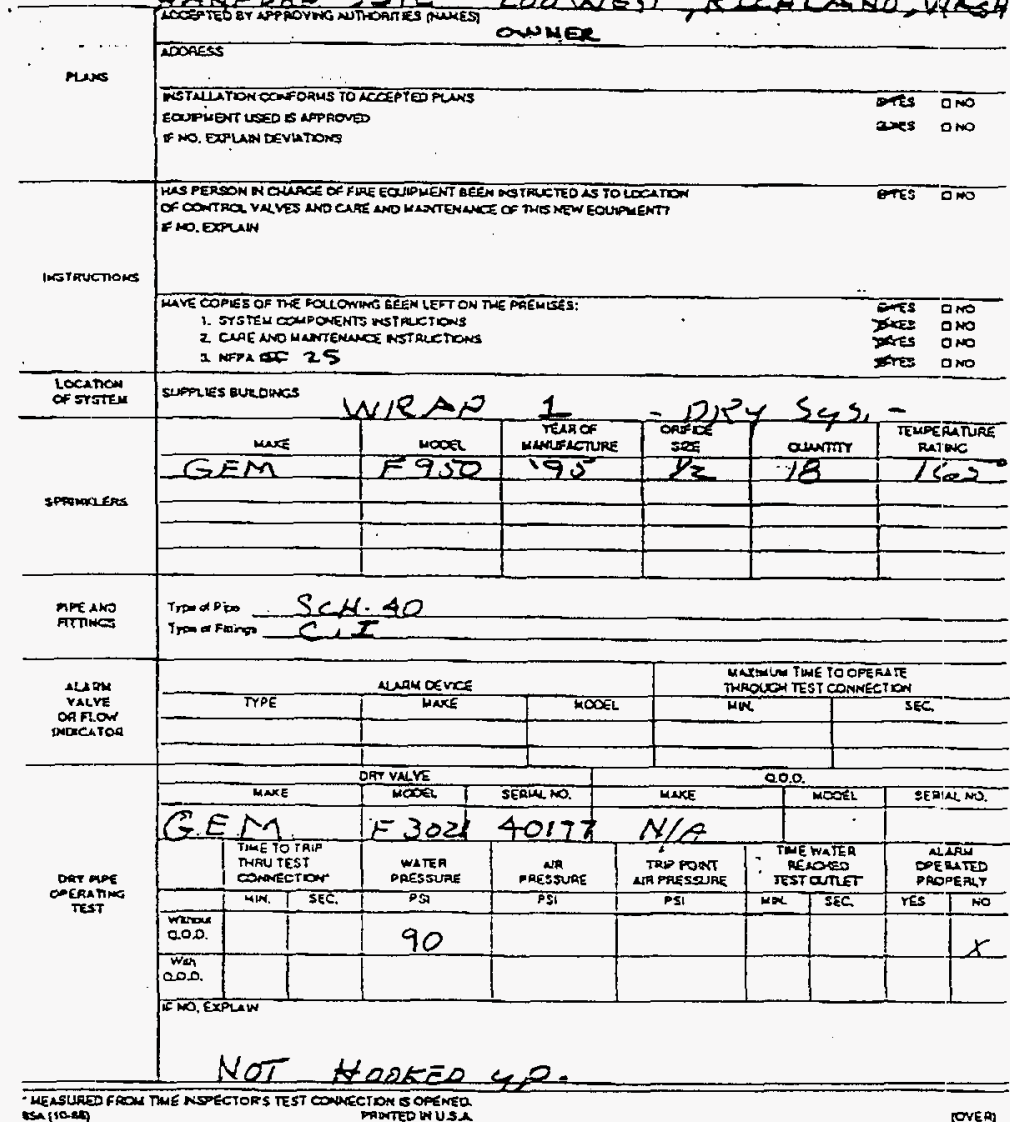

Figure 8-1(a). 


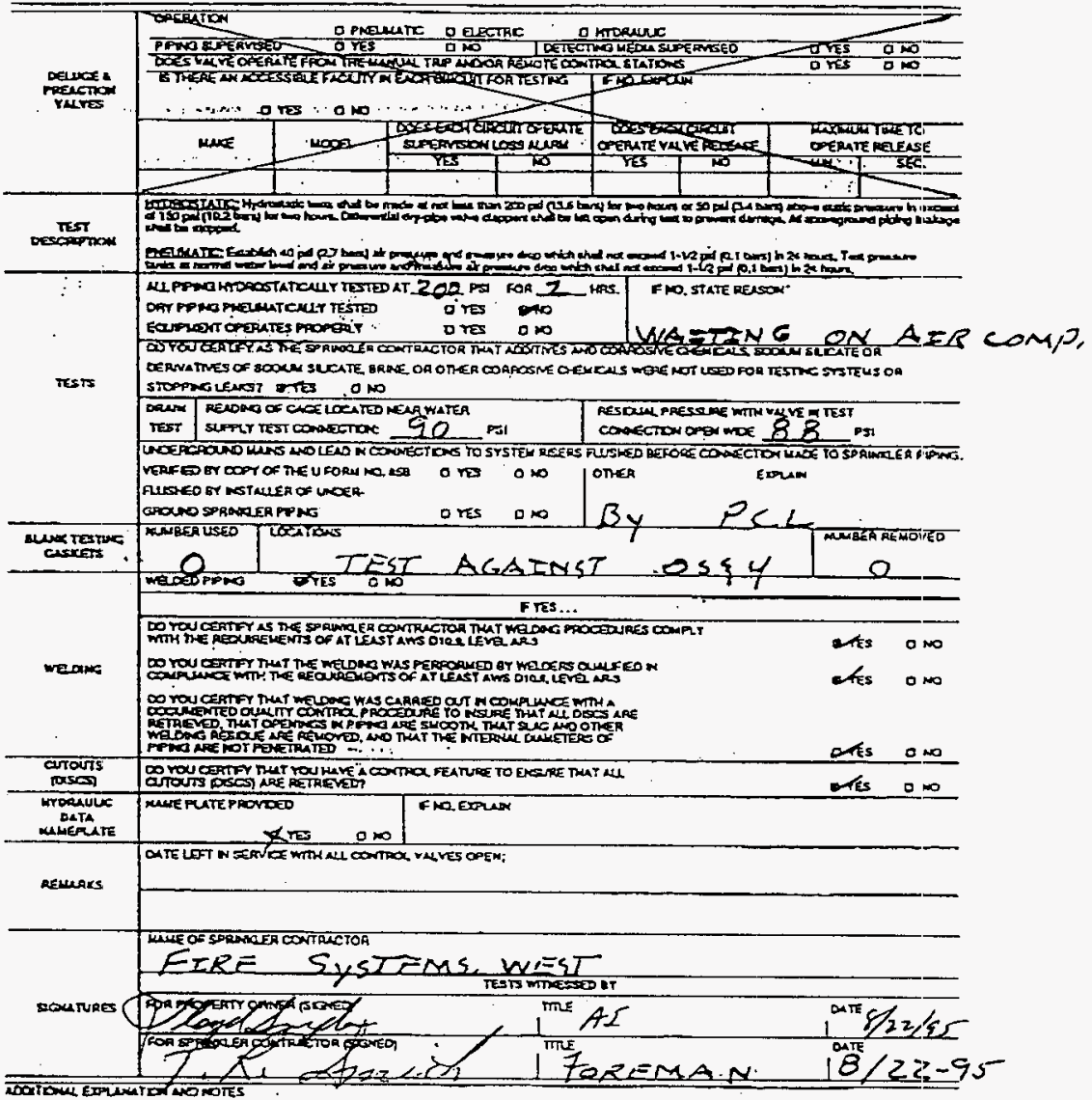




\section{CONTRACTOR'S MATERIAL \& TESTCERTIFICATEFOR A BOVEGROUNDPIPING}

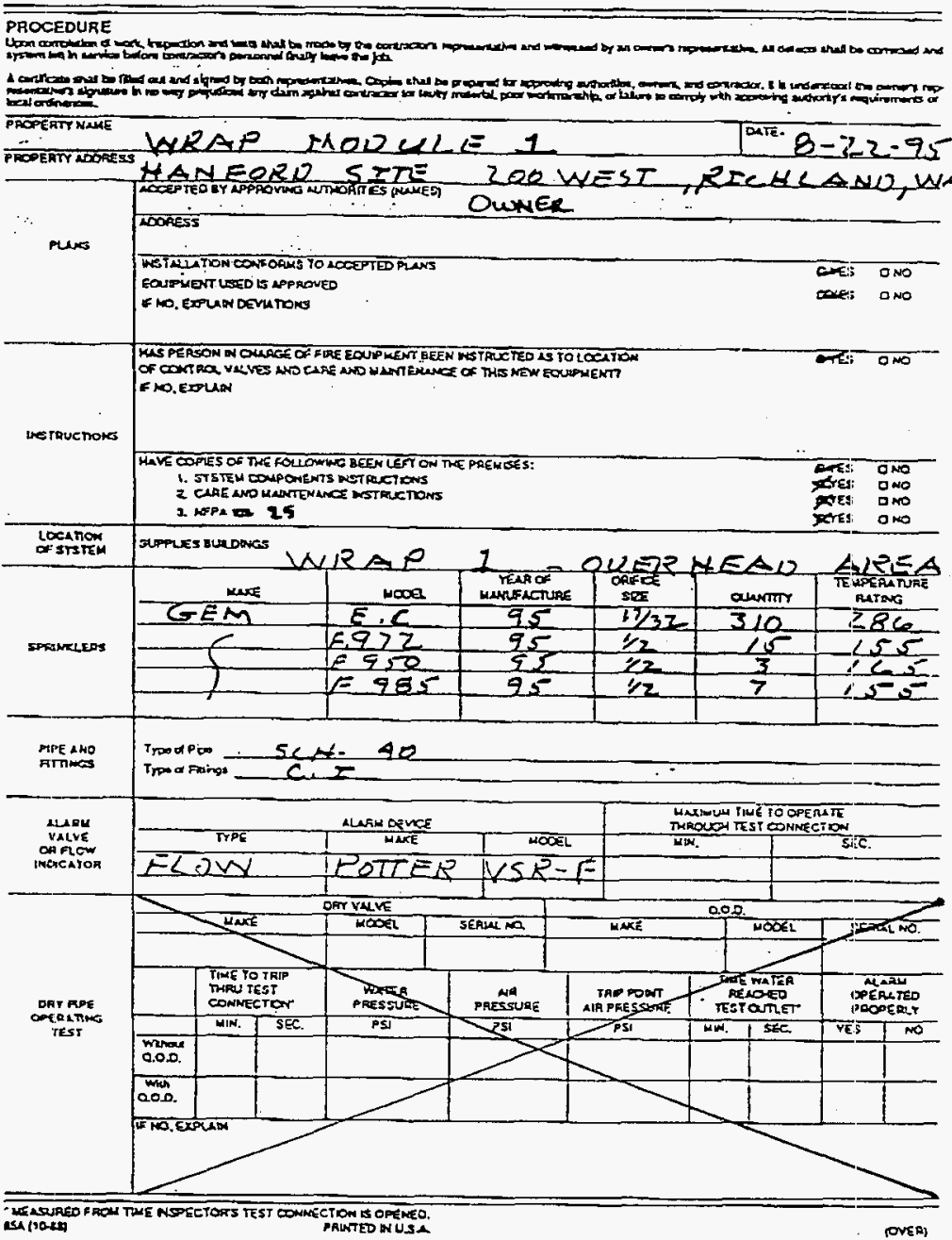

Figure \&-1(a)- 


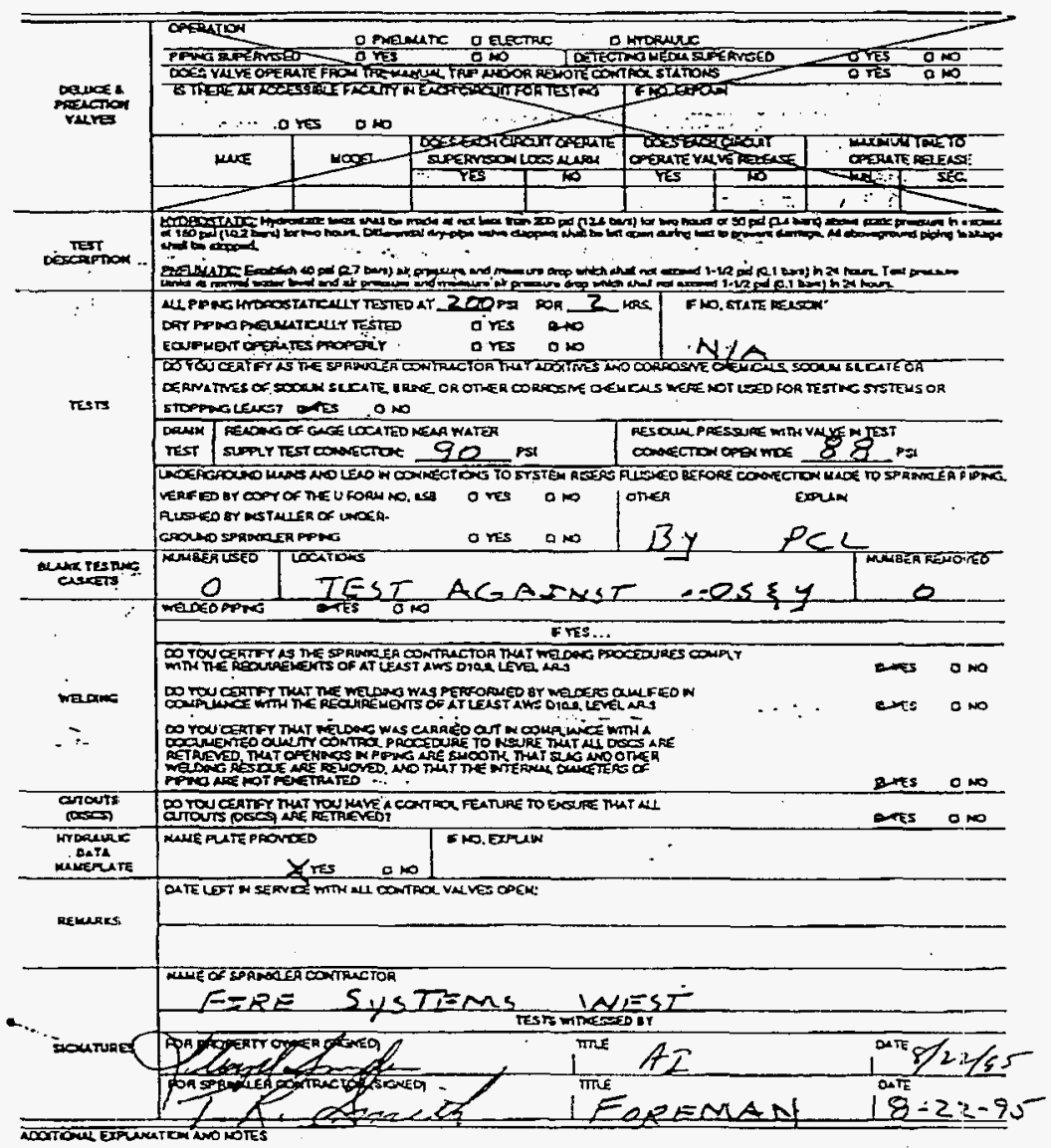

$-\cdots$ 


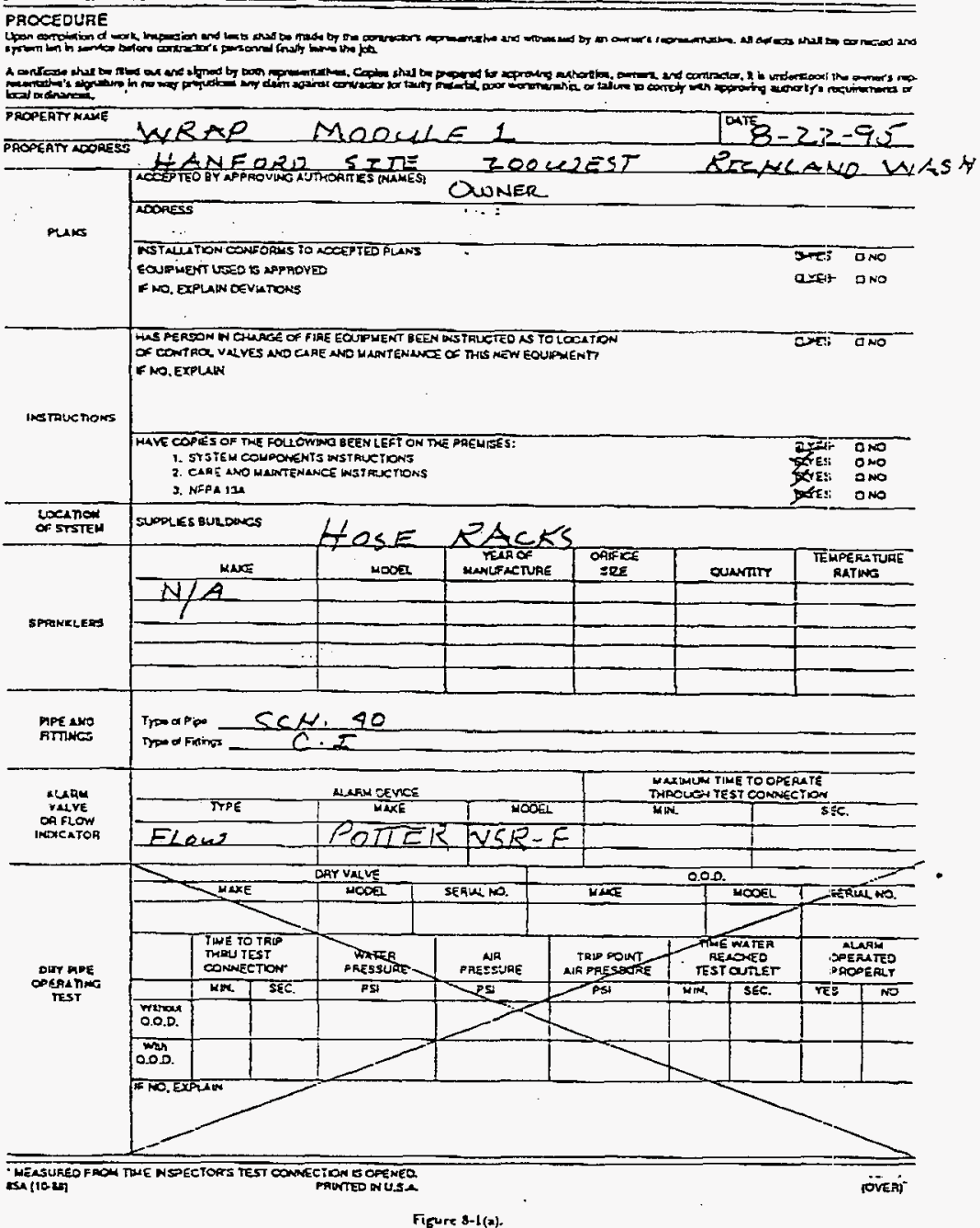




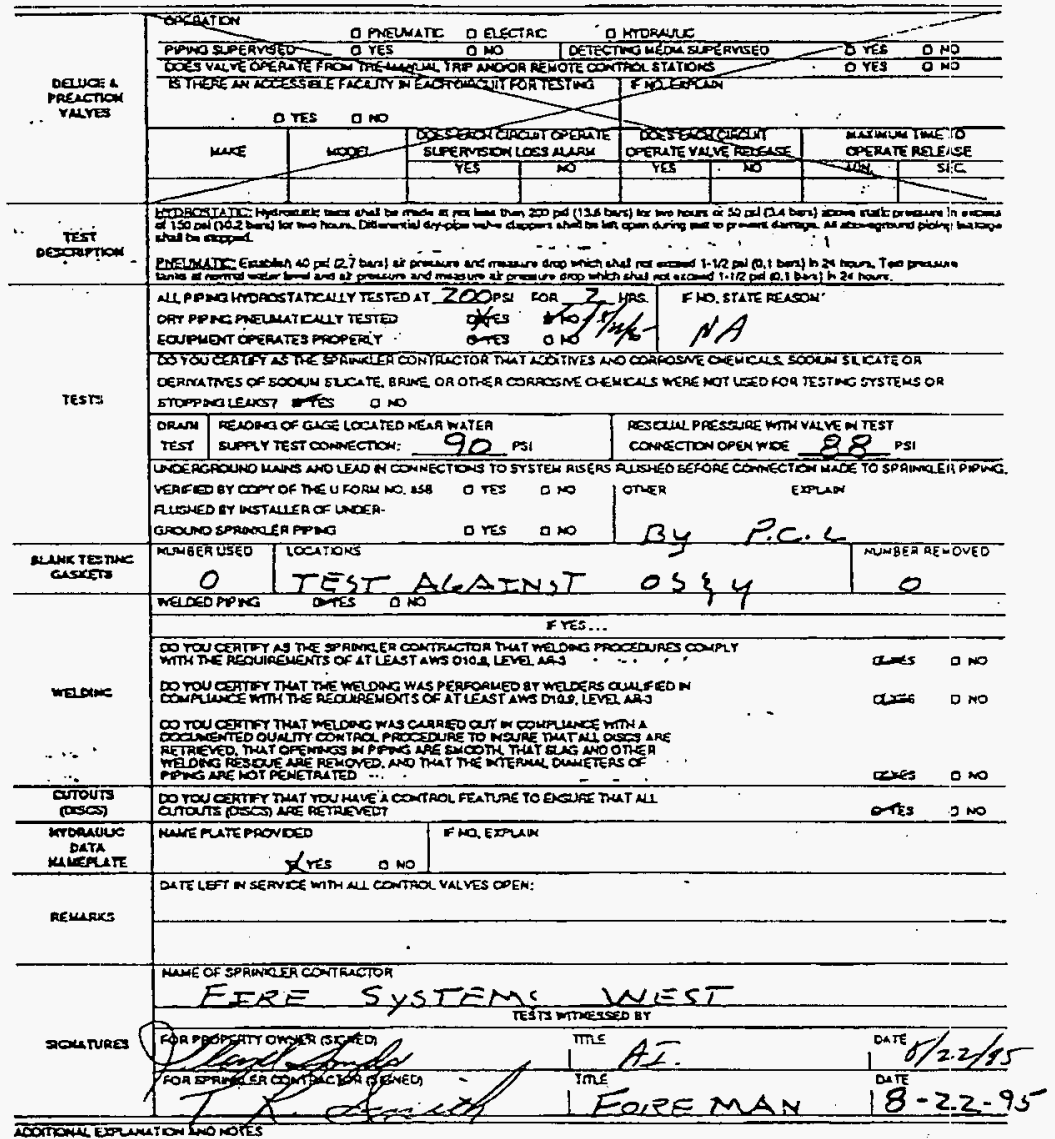




\section{CONTRACTOR'SMATERIAL \& TEST CERTIFICATEFOR $A$ BOVEGROUND PIPING}

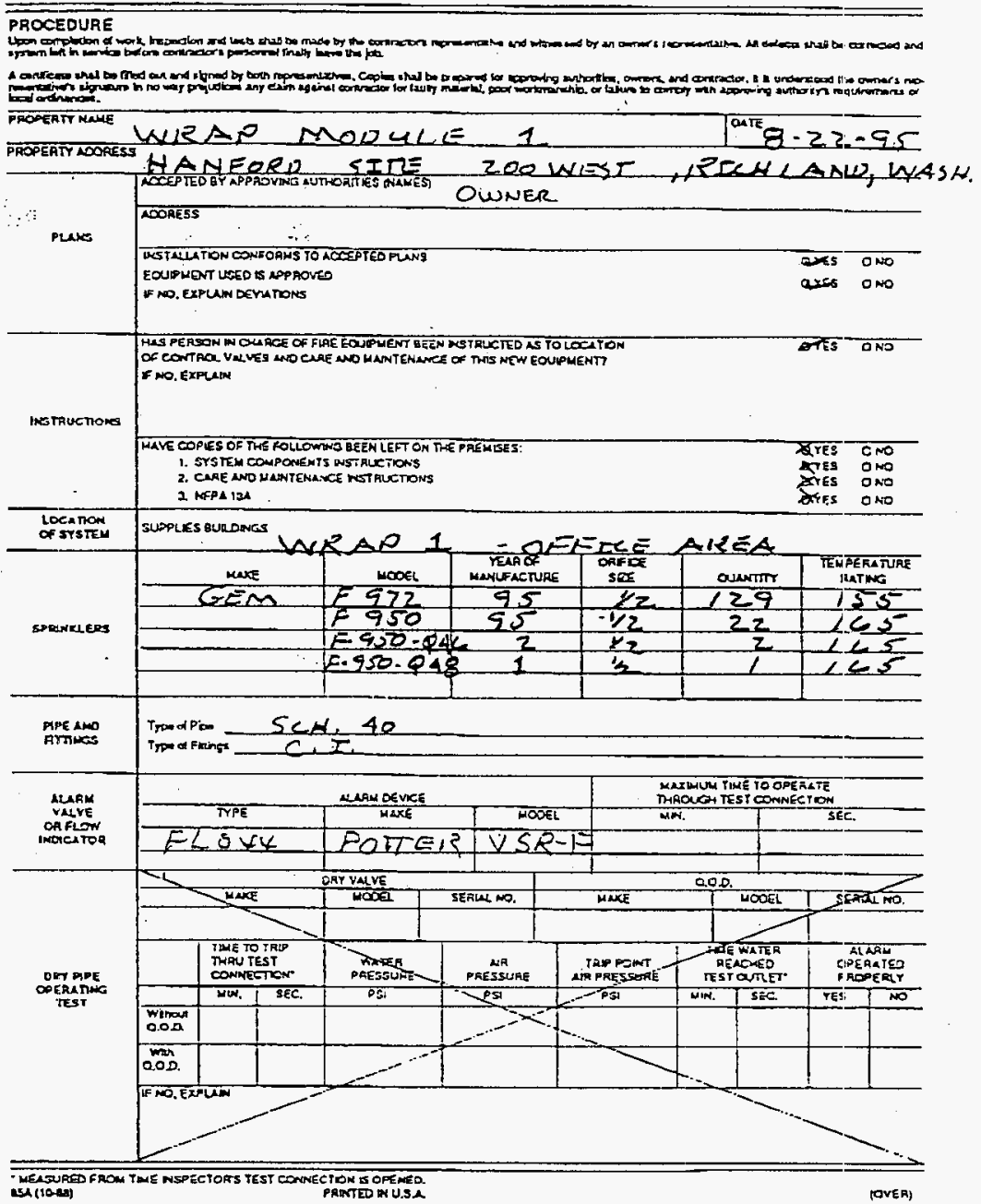

Foure 8-1(x). 


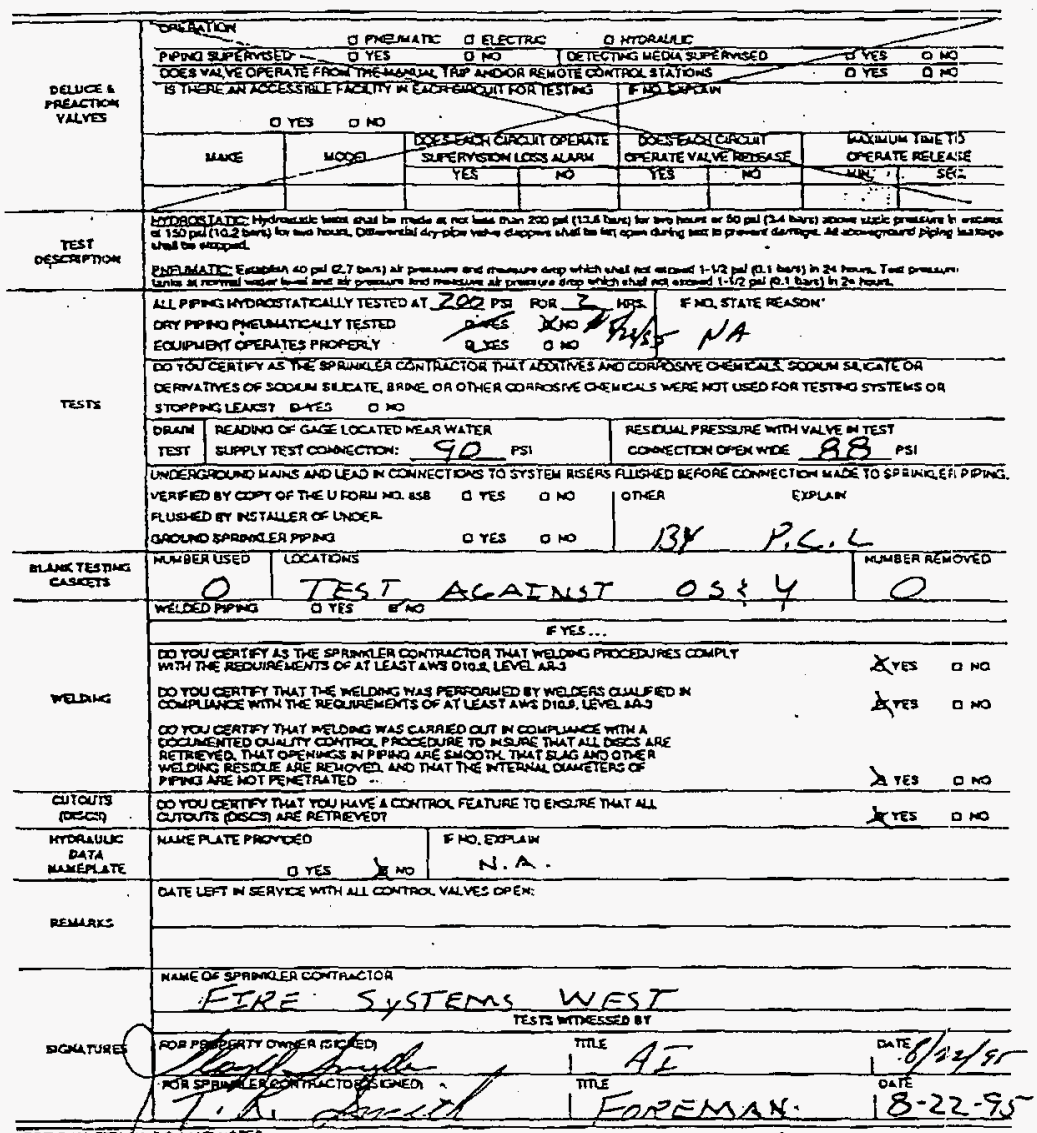

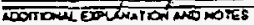




\section{CONTRACTOR'S MATERIAL \& TEST CERTIFICATE FOR UNDERGROUND PIPING}

\section{xaoure}

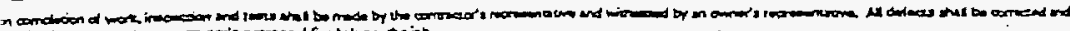

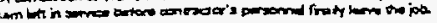

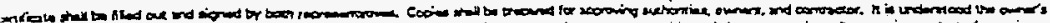

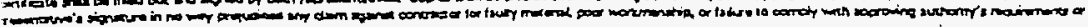
2 arinariat

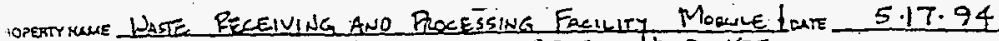

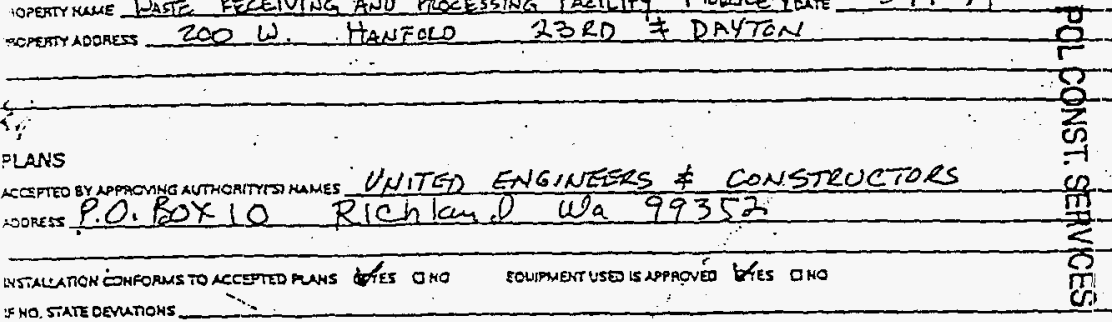

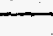

INSTRUCTIONS

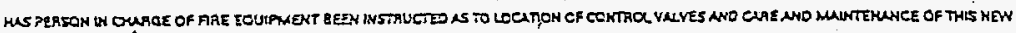

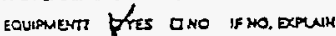

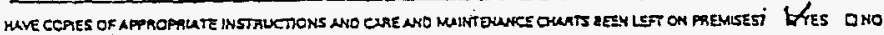

IF NO. DPRWN

LOCATTON

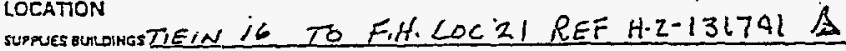

\section{UNDERGROUND PIPES AND JOINTS}

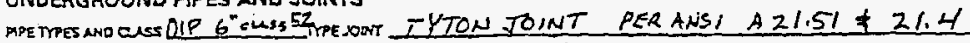

MPE COHFORUS TO

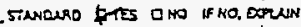

FTTNES CONRORH TO

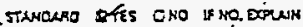

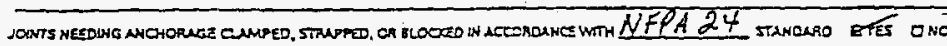
If NO. Borul

\section{TEST DESCRIPTION}

Ruskeve

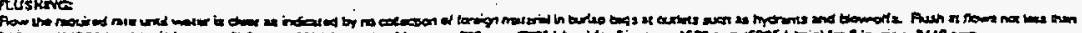

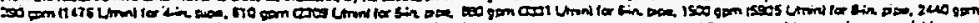

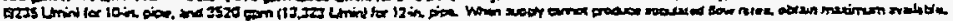

Hromotrance

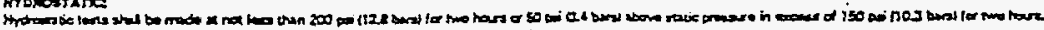
cercus:

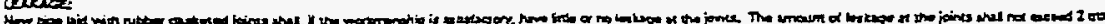

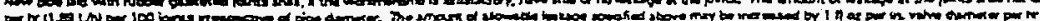

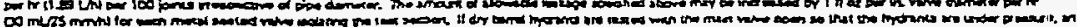

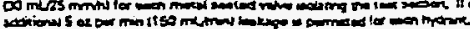

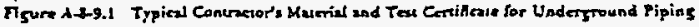




$$
+i 3 / 22 / 96 \quad 13: 47
$$

$8509372 \quad 2131$

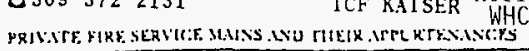

Page 71

FLUSHING TESTS

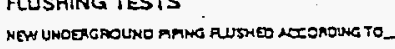

ND Jancha eres atro strousent

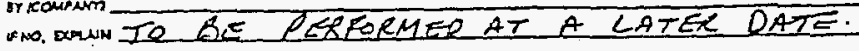

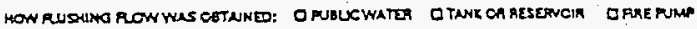

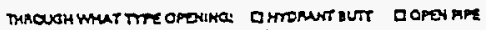

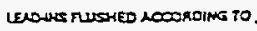
sthound OTES QRo'

Trourum

to NO, DOPANA

HOW RUSHING RLWWASOATANED: D FUEUEWATE DTMKCR RESENOW O ARE PUN

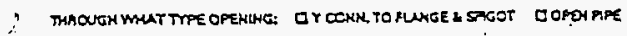

HYOROSTATC TEST

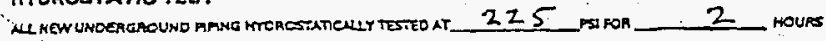

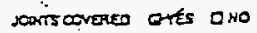

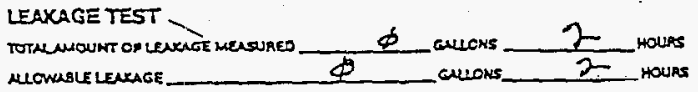

HYDRANTS

MURES WSTALD MPE ANO MANE MENLLLICN ML OPEATE SATSPLTOMLY IFES CNO CONTROL VALYES

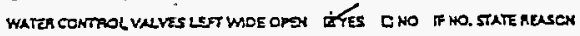

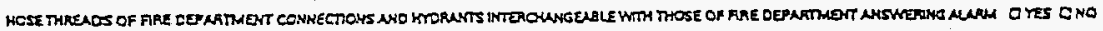

REMARKS

DAR UTT ISRMCE. $5-17-9 ;$ NEMURS

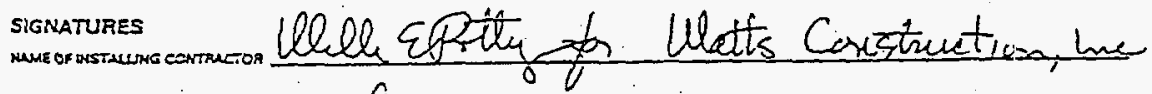

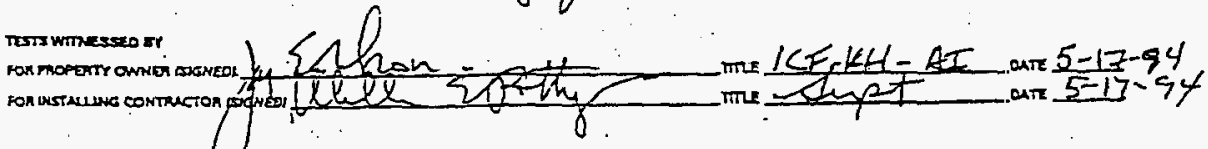

- addTONAL EXPLALUgTion aND NOTES

$$
\text { famen-Noblem (pci) silipas }
$$

Figure A-29.1 leontinnedi.

1932 Estren 


\section{. RECEIVED QNSITE

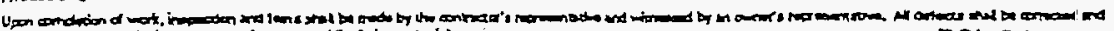

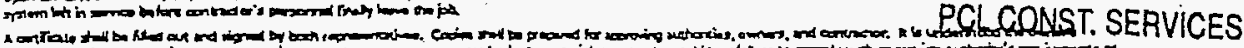

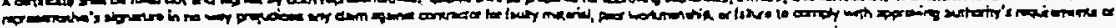

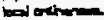

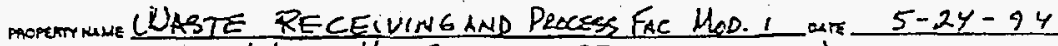
ACRRT ADORES $200 \mathrm{~W}$ HANFORD 23 :D DHYTOR

PLAKS

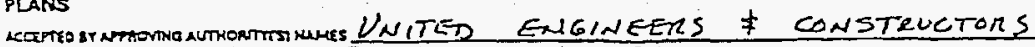
woress P.0. Jox 10 Richland, WA 99352

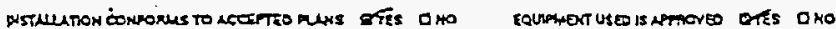
Fia ratr deminous.

\section{IRSTRUCTONS}

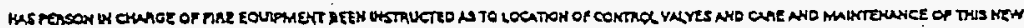

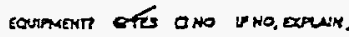

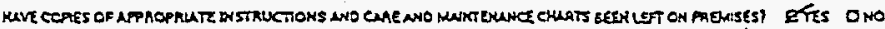
if Mo. Dorus

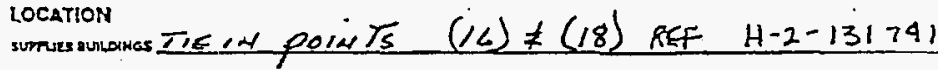

\section{UNDERGROUND PIPES ANO JOINTS}

rrE TrPES NO CUSS TIPE DONT

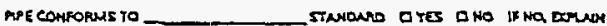

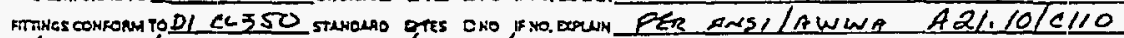

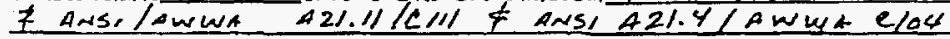

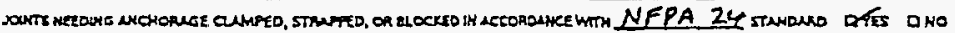
Ho, Expratr

\section{TEST DESCRIP TON}

ruruve

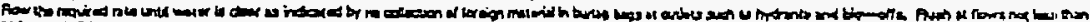

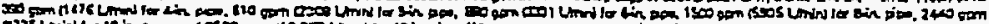

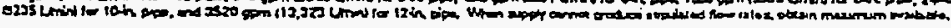
mronortare

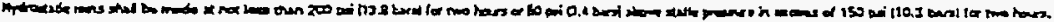
veries:

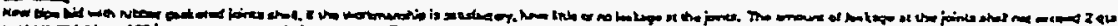

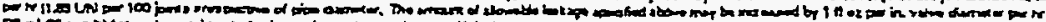

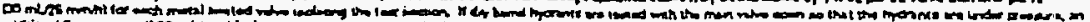

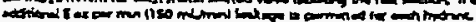




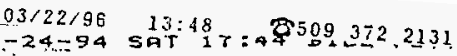

ICF KAISER MOS53

WHC-SD-W026-ATR-013, Rev. 0 Pàge 73

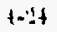

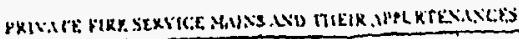

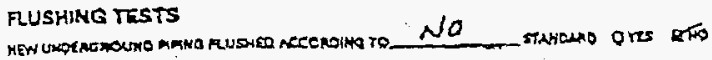

ar compars

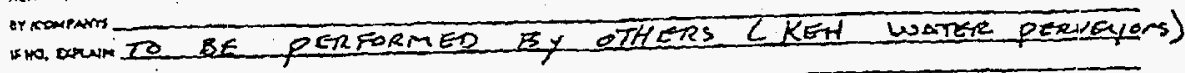

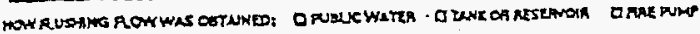

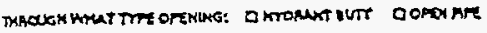

Weins hutorga LCOOHOno to stavena dits DNO

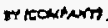

ro, ouvin

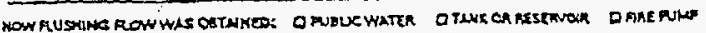

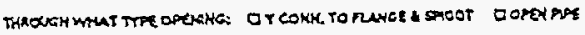

HYDAOSTATIOTEST

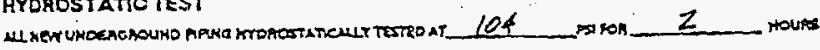
Joms covereo ores ONO

LEACGGETEST (N SERY/LE TEST)

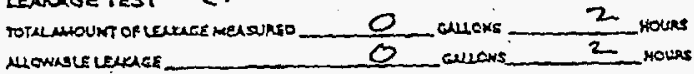

HYORANTS

MLARES INSTALIFO

me ais sux

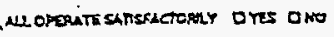

CONTROL VALVES

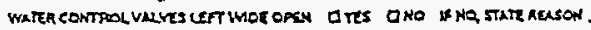

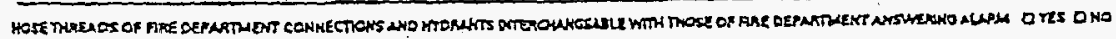

REMARXS

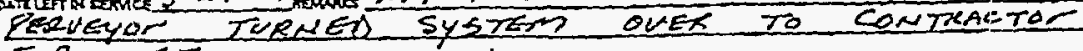
ER USE.

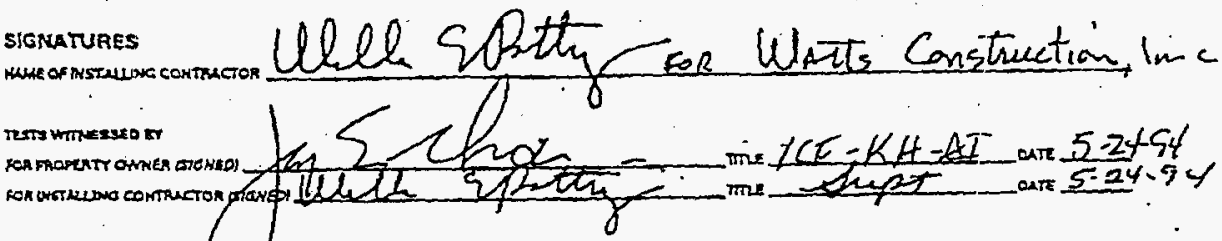

ADOITONAL EXPLAFATIN N ANO NOTES

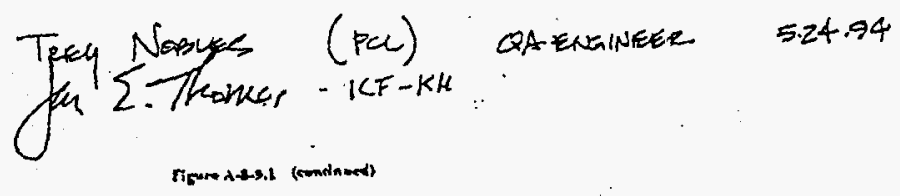




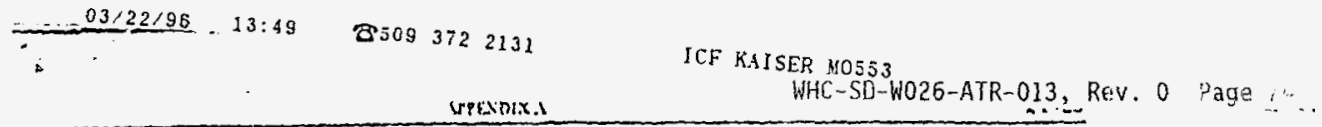

\section{CONTRACTOR'S MATERIAL \& TEST CERTFICATE FOR UNDERGROUND PIFING}

sat

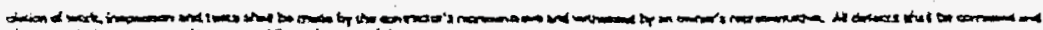

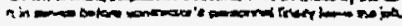

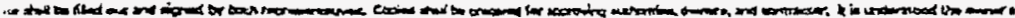

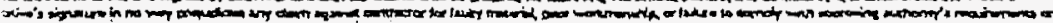
$\sin$

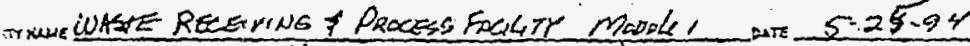

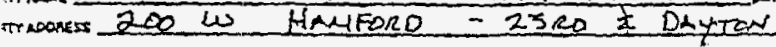

NS

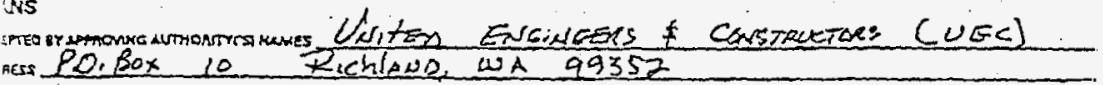

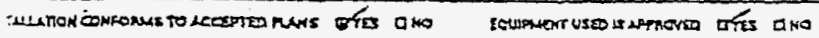

- D. sureomajoks

\section{istRUCTIONS}

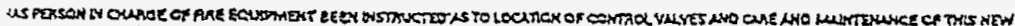

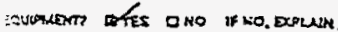

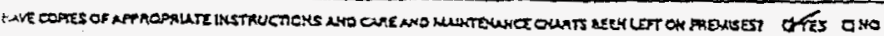

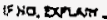

LOCATON

LNOERGROUND PIPES ANO JOINTS

Moriteg arocass D2CLSZ

Dire compons 70

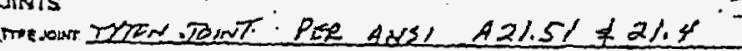

morcscinconth to

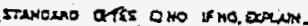

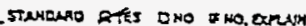

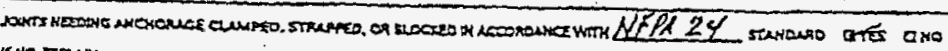
ts Ne, brum

\section{TEST OESCRIPTION}

กUSH

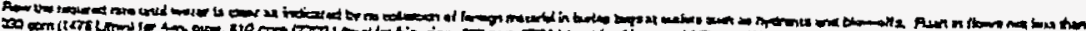

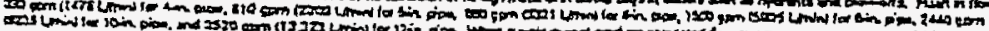
xToertiano

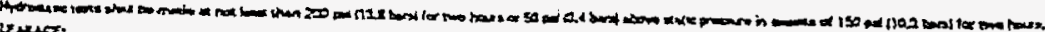
2 Liner.

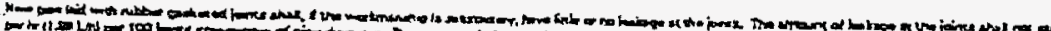

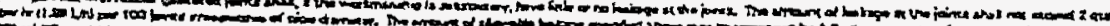

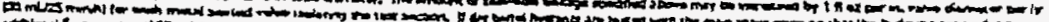

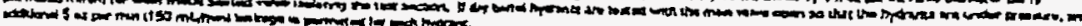

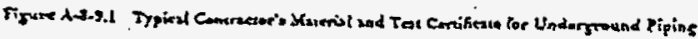


$.03 / 22 / 96-13: 49, \quad-3509 \quad 3 \% 2-2131$

ICF KAISER MO5 53

WHC-SU-W026-ATR-013, Rev. O Page 75

$24-2-1$

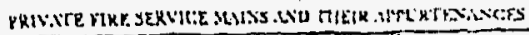

FUUSHING TESTS

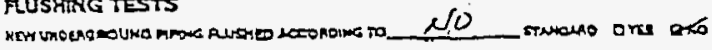

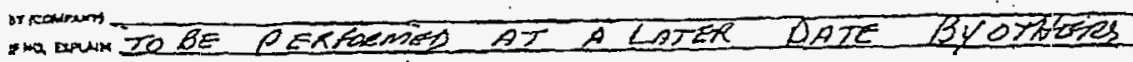

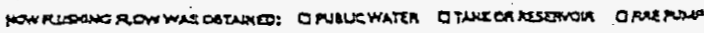

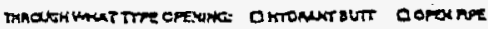

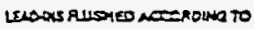
struns ares ano

roceupam

NKO, CONW

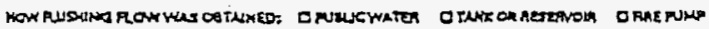

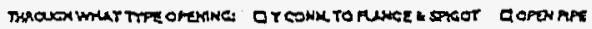

HYOROSTATICTEST

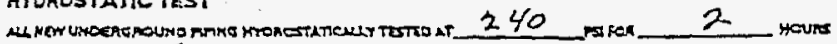
Jensomores ofis ono

$\because$ LEAKaGE TEST

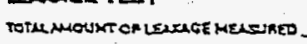

MOWLALLELTER

Q

O awons

HYRRANTS

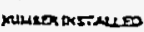

menone elON (MEDACLON)

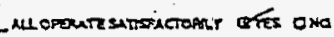

CONTROL YALYES

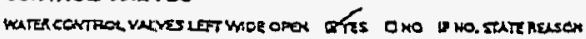

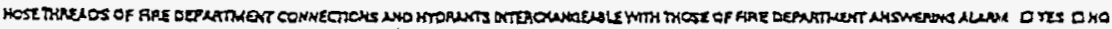

REMARXS

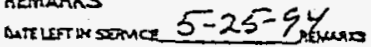

SIGMATURES

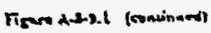




\section{acoura}

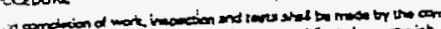

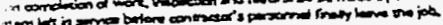

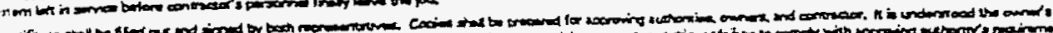

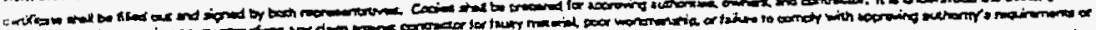

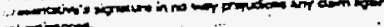

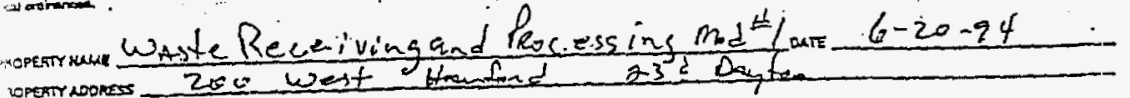

FILE:

$02-09-90$

$C C: D L$

?LANS

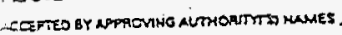
PDE $2 C$ Kulated $w_{k}$ 99352 NDORESS COUPMENT USE IS APMOVED. DTES ONO

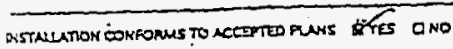

IF Na. STATE DEMATIOKS

$\because \therefore$

\section{INSTRUCTIONS}

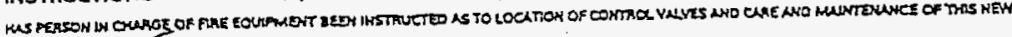

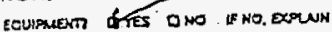

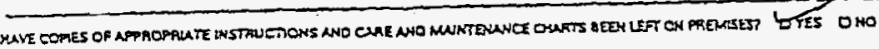

IF NO, DOWN

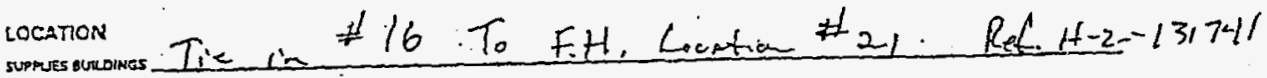

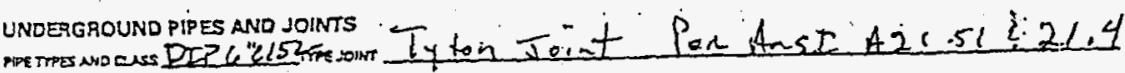
PTPE COMFORYS :O

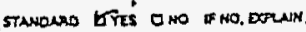

FITNES CONFORM TO

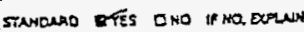

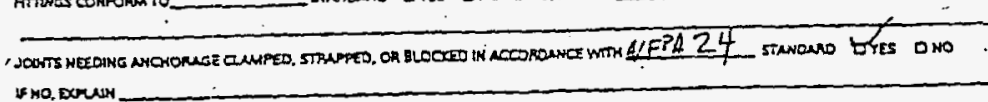

\section{TEST DESCRIPTION}

nuskme

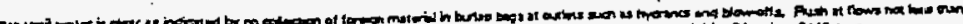

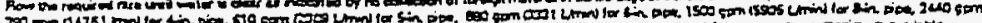

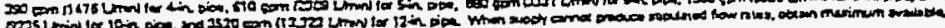

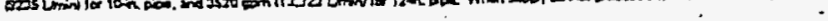

Mratitie

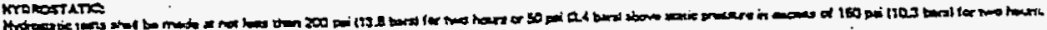

Ueves:

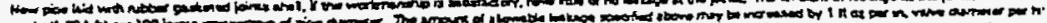

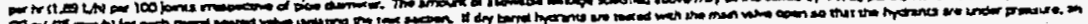

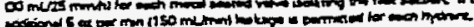

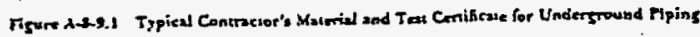




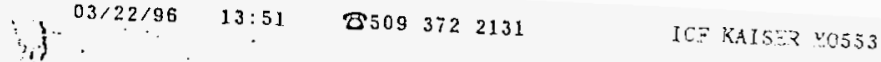

$$
\begin{aligned}
& 24-24
\end{aligned}
$$

FLUSHING TESTS

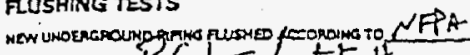

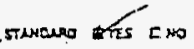

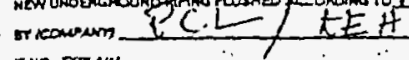

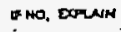

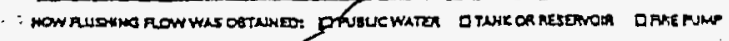

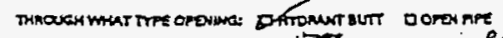

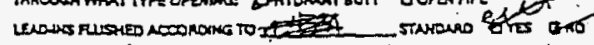
or trowents

IFO. Dormin

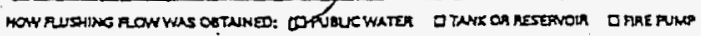

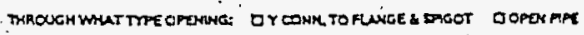

HYDROSTATIC TEST

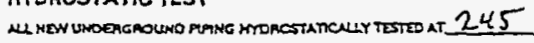
$\rightarrow$ ron Mours Jams COVERS D TES ONO

LEAKAGE TEST

TOTN MOANT OF LACAGE MEISURED

$p$

gullows HOUns

NLONABLE LENGCG acuens Hours

HYDRANTS

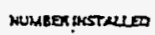
menta mua Medolion.

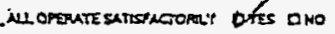

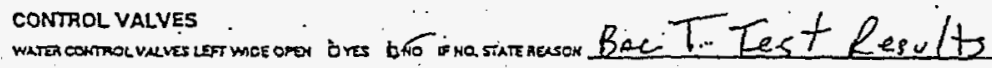

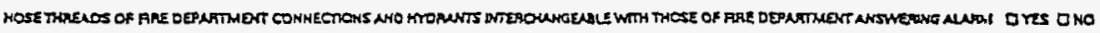

REMARKS

- ORTE LET W SEMTE Neveris

SIGNATURES

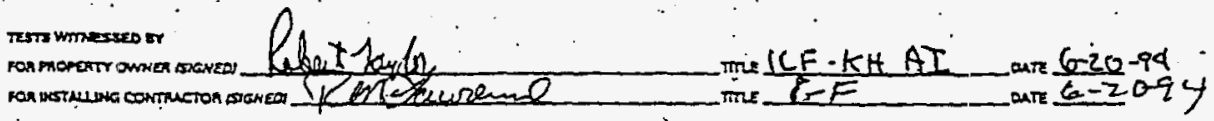

ADOTTONAL EXPLANATION AND NOTES

1992 Edtion 
rocesurat

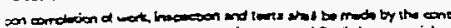

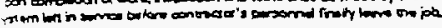

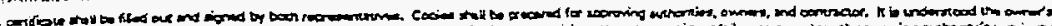

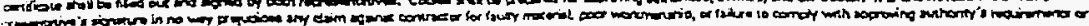
alocinares

guts 8.18 .94

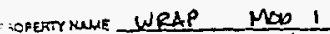

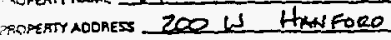

PLANS

ACCRTED BY APARCNHG AUTHOMITTS RAMES UE $U C$

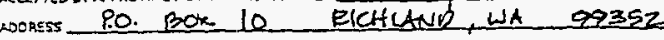

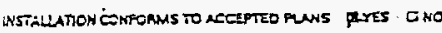

ECUIAMENT USED IS APPROVED FARS QNO

UNO STATE DENATIONS

INSTRUCTIONS

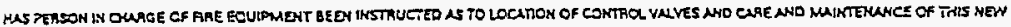

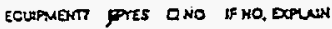

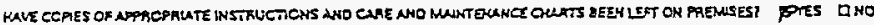

IF NC. DOPUNK

LOCATION

$$
4^{\prime \prime} \text { POTABUE }
$$

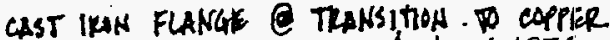

SUPRUES BULOTHES TIE IN H 23 to

REF

H. $2 \cdot 1317416$

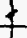

181874

UNDERGROUND PIPES ANO JOINTS

PPE TTES NDOUSS DP

MPE CONFORMS TO

FTnNeS CONFORM TO

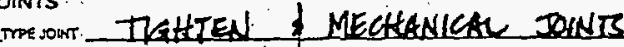

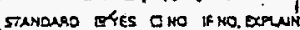
STANOARO OfES ONO IF NO. DXMaH

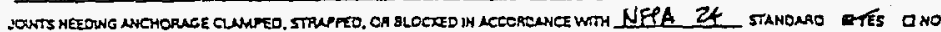

FF HO. STMUN

\section{TEST DESCRIPTION}

\section{Ruskine}

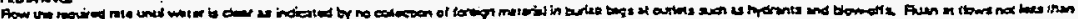

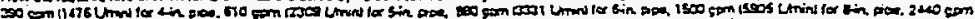

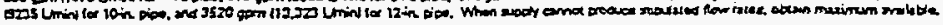

HYonostine

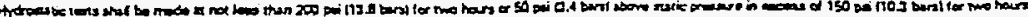
Lexces:

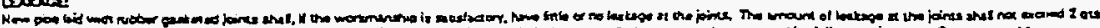

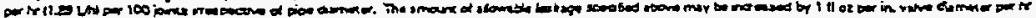

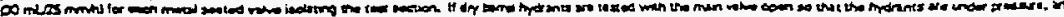

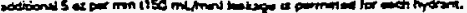

Fyore A-t-9.1 Typieal Contector's Mulerial snd Test Cenifeste for Underspound Piping 
$+$

\section{FUUSHING TESTS}

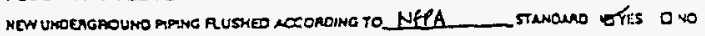

DT cowrom

F He. DPewk

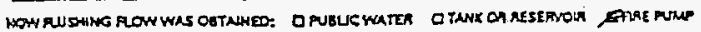

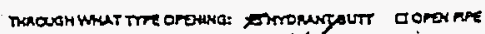

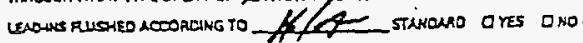

or reowpent?

$-$

WO. DONN

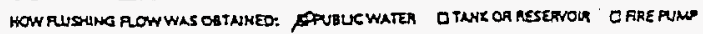

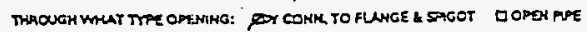

\section{HYDROSTATICTEST}

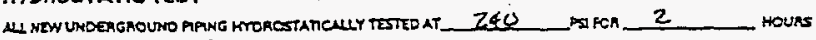

Jants coverso unes firo

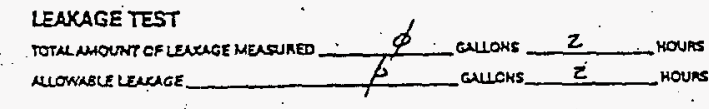

HYDRANTS

MUMEg IRSTNLE - Troma ware NLSOEUTE SUTSFATORLY O TES QNO

\section{CONTROL VALVES}

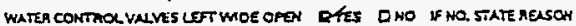

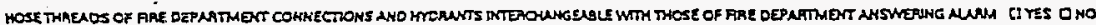

REMARKS
OUE LET SEMCE $0 / / 8 / 94$ Rourus

\section{SIGNATURES}

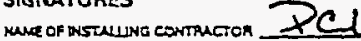

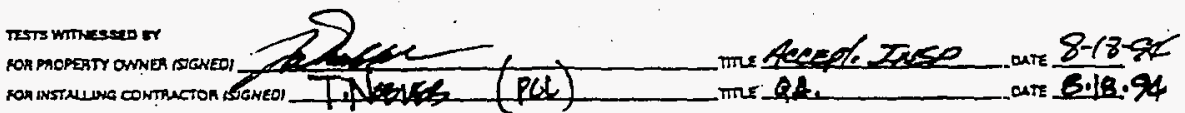

ADOITIONAL EXPLANATION AND NOTES

Ficture A+s.9.1 (esatinund) 
03/22/96 13:52 B509372 2131 ICF KAISER MO553

IPPEIBIX.1

$24-23$

WHC-SD-W026-ATR-013, Rev. 0 Page 80

CONITACTORS MATERIAL \& TEST CERṬFICATE FOR U NDERGROUND PIPING

mocouse

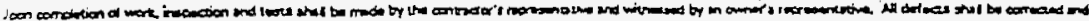

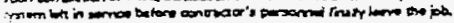

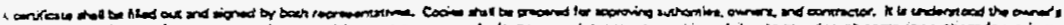

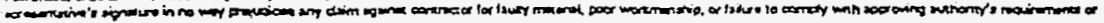

bolorinane

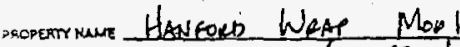

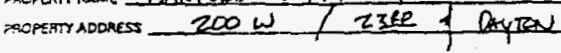

arte

$9 \cdot 7 z \cdot 94$

PLANS

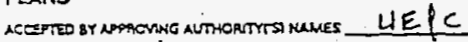

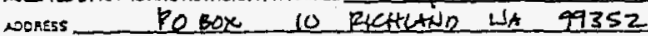

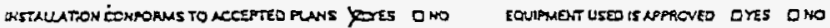

If NO, STRTE Dematows

INSTRUCTIONS

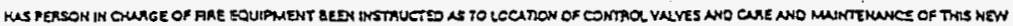

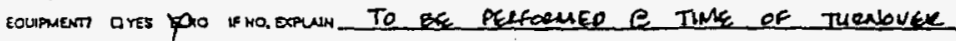

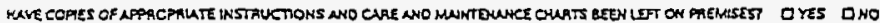

LF NO. DXFUM

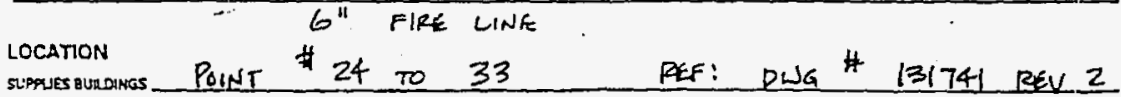

UNDERGROUND PIPES ANO JOINTS

PDE TrRes ANQCUNS DIP

PIPE CONFORMS TO NEPA

RTMNGS CONFORM TO $\triangle$ FPP

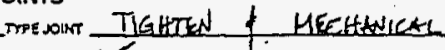

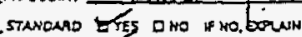

STANOANO TES anO IENO, DXMAN

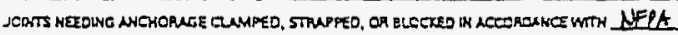

STANDNA CYES DNO

IF NO, EOPUN

\section{TEST DESCAIPTION}

ruskinas

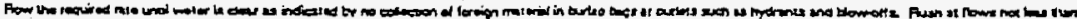

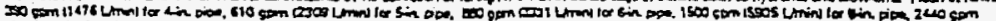

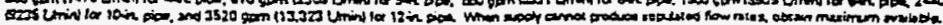

Mronotrato:

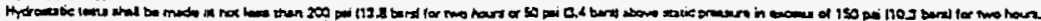

Letruac:

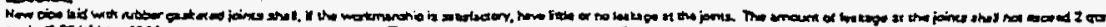

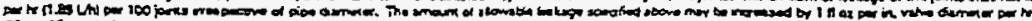

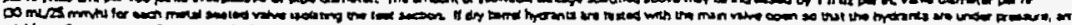

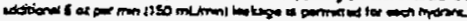

Fipure d-8-9.1 Typical Conszacior's Mverial and Tenl Cerrifieste for Underground Piping

RECEIVED ONSITE W.R.A.P.\#1

AlIG 241994

1092 Exison

PCL CONST. SER

FILE

T. SERVICES

3.5 


\section{FUSHING TESTS}

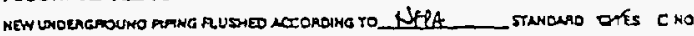

ro conew

Fro. Extwh

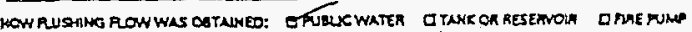

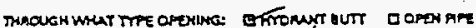

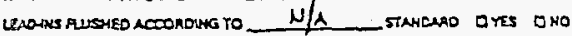

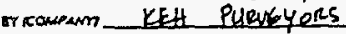

* Ho. Dorus

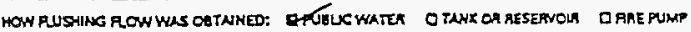

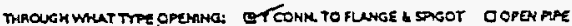

HYDROSTATTC TEST

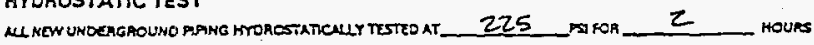

SONTS COVERED D TES OTO

\section{LEAKAGE TEST}

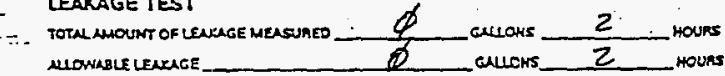

HYORANTS

WMEER AKSTMU⿴囗十 me NWD Mure

\section{CONTROL VALVES}

WATER CONTRO VMVVESIET MOEORE ETES ONO IF NQ STLERESON

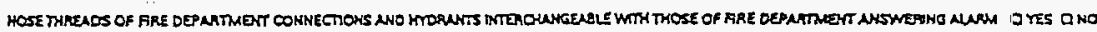

REMARKS

\section{SIGNATURES}

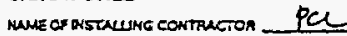

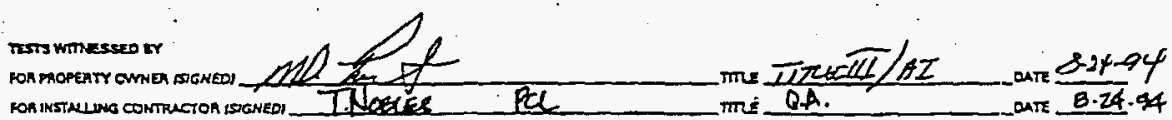

ADDMONAL EXPLANATION AND NOTES

WITNESSED FULSHINA:

TWhates. PCL. B.24.94.

Figrod A-9.1 (condinat) 


\section{CONTRACTOR'S MATERIAL \& TEST CERTIFICATE FOR UNDERGROUND PIPING}

\section{nocrouse}

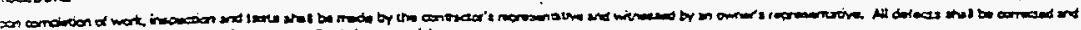

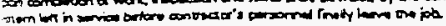

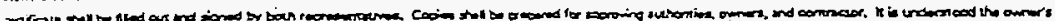

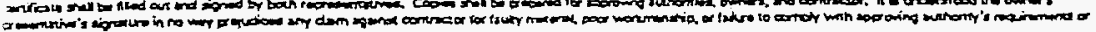
sicritinat.

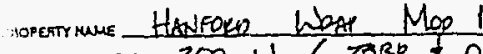
ant 8.22 .94

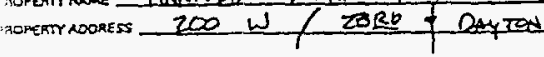

\section{gLaNs}

A.CEPTEO BY NOPAOVHG AUTHCAITTS NUMES UE $4 C$
ADORESS
Po Eox
10
LICHLANO WA

\section{2}

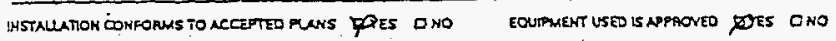

IF NO, STARE OEVMTHONS

\section{INSTRUCTIONS}

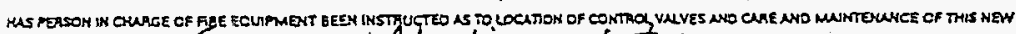

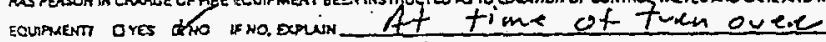

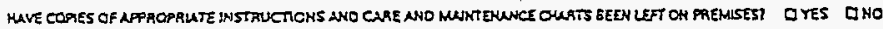

iF HO, ExNA

$$
\text { 4* IRRIGATION }
$$

LOCATION

supputs einloings POINT \# 23 TO 36 REF: DWG \#131741 2EV.2

\section{UNDERGROUNO PIPES AND JOINTS}

PISETPES ANOANS DIF

RPE CONFORMS TO $\sim$ EPA

ПTINAS CONFORM TO NIEPA
TrPe JOLit

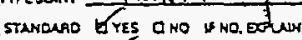
sthicaro efes ano if yo. Domuin

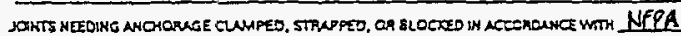
surosio efes ano

if NO. EXPLWN

\section{TEST DESCRIPTON}

Tusmera

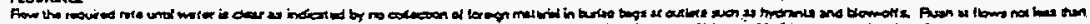

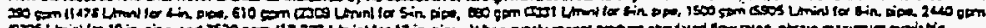

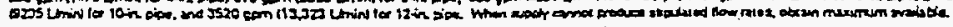

Mrokostatk

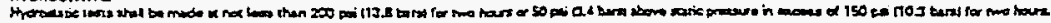

Herces:

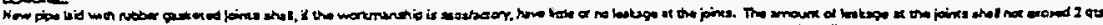

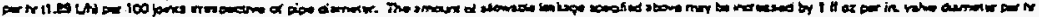

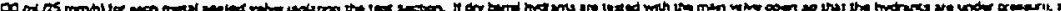

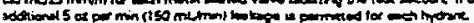

Finure ג-8.9.1 Typiesl Contrator's Matrial and Tet Certikeale for Underground Piping 


\section{FLUSHING TESTS}

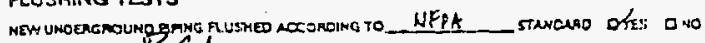
ar compum $C$

ano, Dorun

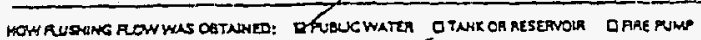

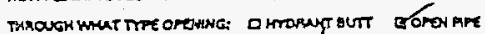

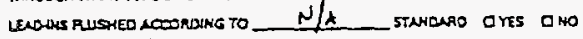

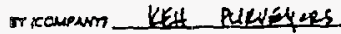

If Mo. Expun

HOW RUSHAG FLOWWAS OBTAMED: WUBUSWATER DTAKKCARESERVOR ORAEFUM

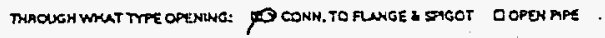

\section{HYDROSTATICTEST}

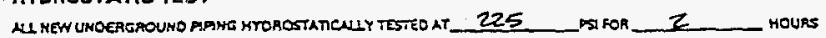

Jants COVERE D TES Yoro

\section{LEAKAGE TEST}

-. TOTL MUOUNT OF LECAGE MERSURED HLWOWBLE LEAGE $\frac{2}{2}$ cunons mours

\section{HYDRANTS}

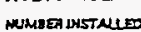
$\phi$ TrPE MNO MUAS

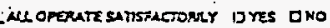

CONTROL VALVES

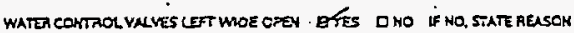

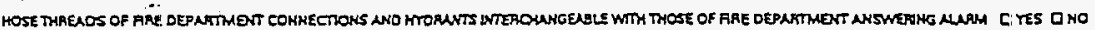

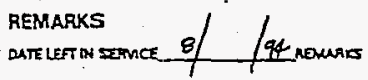

\section{SIGNATURES}

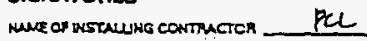

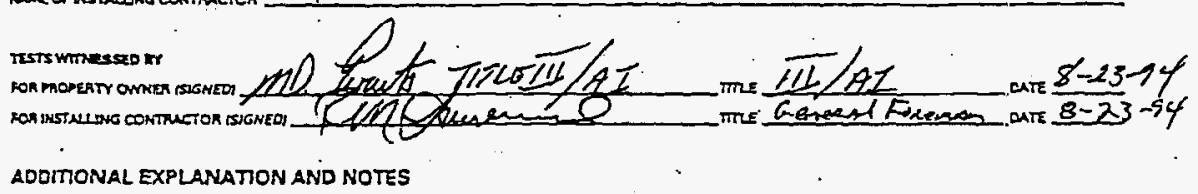

ADDITONAL EXPLANATION AND NOTES

WITESS Flush: Theues (pel) \&.16.94

Bgrare At-9.1, (eoncisurd) 
DON'T SAY IT --- Write It!

To: Larry Anderlini

cc: DJ Hart
DATE: May 2, 1996

WHC-SD-WO26-ATR-013, Rev. 0 Page 84

FROM: Valerie S. Mitchell

SWD Industrial Hygiene

Telephone: $372-3932$

SUBJECT: WRAP 1 FIRE ALARM SOUND LEVEL TESTS

Per your request, on May. 1, 1996, I conducted a sound survey at the WRAP 1 facility during the ATP for the facility's fire alarm bells.

A Quest Model 2800 Impulse Integrating Type II Sound Level Meter (serial \# HS5080028, IHEL \#N018) was used to measure the sound levels. The instrument was factory calibrated, traceable to NIST standards, on $9 / 26 / 95$ and is due for factory re-calibration by $9 / 26 / 96$. The meter was also field-calibrated, per the manufacturer's instructions, immediately prior to and after use- with a Quest QC-10 Acoustic Calibrator (serial \# QE5090032, IHEL \# NC20). The QC-10 calibrator was calibrated by the WHC Industrial Hygiene Equipment Laboratory (IHEL) on $9 / 26 / 95$ and is due for re-calibration by $9 / 26 / 96$. The pre-and postjob calibrations were $114.1 \mathrm{dBA}$, acceptably close to the $114.0 \mathrm{dBA}$ noise leve] produced by the calibrator. During the survey, the meter was set for silow response on the A-weighted scale, as is standard for surveys of this type and as recommended by WHC-IP-0030, section.

The survey results are summarized in the following table. Both the ambient" and "During" levels noted were averages for the room/area.

\begin{tabular}{|c|l|c|c|}
\hline \hline ROOM \# & ROOM DESCRIPTION & $\begin{array}{l}\text { AMBIENT (PRE-ALARM) } \\
\text { NOISE LEVEL (dBA) }\end{array}$ & $\begin{array}{c}\text { (DURING) ALARM } \\
\text { NOISE LEVEL (dBA) }\end{array}$ \\
\hline 101 & shipping/receiving & 69 & 80 \\
\hline 102 & s \& r office & 64 & 64 \\
\hline 103 & office & 69 & locked \\
\hline 104 & NDE/NDA & 68 & 79 \\
\hline 107 & process area & 70 & 74 \\
\hline 109 & sample management & 71 & 65 \\
\hline 113 & process HVAC & 70 & 81 \\
\hline 116 & switchgear room & 72 & 72 \\
\hline 118 & mechanical room & 92 & N/A \\
\hline 120 & RPT room & 64 & 64 \\
\hline 121 & storage & 64 & 64 \\
\hline 122 & RCD management & 63 & 63 \\
\hline 123 & operations mgr & 63 & 63 \\
\hline 125 & secretarial & 65 & 79 \\
\hline
\end{tabular}




\begin{tabular}{|c|c|c|c|}
\hline ROOM \# & ROOM DESCRIPTION & $\begin{array}{l}\text { AMBIENT (PRE-ALARM) } \\
\text { NOISE LEVEL (dBA) }\end{array}$ & $\begin{array}{l}\text { (DURING) ALARM } \\
\text { NOISE LEVEL (dBA) }\end{array}$ \\
\hline 126 & reception/waiting & 66 & 85 \\
\hline $128 / 129$ & conf/lunch room & 64 & 64 \\
\hline 124 & RPT & 66 & (locked) \\
\hline 131 & soiled laundry & 66 & 66 \\
\hline 131 & clean laundry & 65 & 67 \\
\hline 134 & storage & 65 & 72 \\
\hline 136 & storage & 67 & 68 \\
\hline 139 & equip. maint. & 63 & 64 \\
\hline $140 / 141$ & women's locker & 66 & 66 \\
\hline 144 & women's SWP/PCM & 65 & 65 \\
\hline 146 & men's SWP/PCM & 66 & 66 \\
\hline $148 / 150$ & men's locker & 66 & 66 \\
\hline 149 & men's shower & 67 & 68 \\
\hline 152 & material prep. & 64 & 64 \\
\hline $\begin{array}{l}\text { North } \\
\text { stairwell }\end{array}$ & -non--o- & 65 & 67 \\
\hline $\begin{array}{l}\text { South } \\
\text { stairwell }\end{array}$ & -...-- & $64^{\circ}$ & not taken \\
\hline 201 & control room & 66 & 76 \\
\hline $201 \mathrm{~A}$ & control office & 68 & 71 \\
\hline 202 & computer room & 66 & 76 \\
\hline 203 & mechanical room & 69 & 75 \\
\hline
\end{tabular}

In six of the rooms/areas -- 101, 104, 113, 125, 126, and 201 -- the sound levels measured during the alarm were at least $10 \mathrm{dBA}$ higher than the "ambient" (pre-alarm) levels. In the remainder of the rooms/areas, the noise level difference was less than the $10 \mathrm{dBA}$ difference specified in the WRAP 1 FDC, SDC 7.8.

It is important to note that the "ambient" levels observed were those present at the time of the survey and are not necessarily indicative of what sound levels will be when the facility is operational and/or occupied.

If you have any questions, please call me at 372-3932 or stop by to see me. 
DON'T SAY IT -.- Write It!

TO: Larry Anderlini

cc: DJ Hart

RJ Koll
WHC-SD-W026-ATR-013, Rev. 0 Page 86

DATE: June 3,1996

FROM: Valerie S. Mitchel

SWD Industrial Hygiene

Telephone: $372-3932$

SUBJECT: WRAP 1 FIRE ALARM SOUND LEVEL TESTS CONDUCTED ON 6/3/96

Per your request, on June 3, 1996, I conducted a sound level survey at the WRAP 1 facility during an ATP for the facility's audible fire alarm devices. These devices were installed to replace the fire alarm bell system previously tested on $5 / 2 / 96$ (see DSI, VS Mitchell to L. Anderlini, dated 5/2/96).

A Bruel \& Kjaer Model 2231 Modular Precision Integrating Type I Sound Level Meter (serial \# 1735186, IHEL \#N007) was used to measure the sound levels. The instrument was factory calibrated, traceable to NIST standards, on 1/08/96 and is due for factory re-calibration by $1 / 07 / 97$. The meter was also field-calibrated, per the manufacturer's instructions, immediately prior to and after use with a Bruel \& Kjaer Type 4231 Acoustical Calibrator (serial \# 1795583, IHEL \# NC14). The 4231 calibrator was calibrated on $1 / 08 / 96$ and is due for re-calibration by $1 / 07 / 97$. The pre-and post-alarm calibrations were $94.2 \mathrm{dBA}$, acceptably close to the $94.0 \mathrm{dBA}$ noise level produced by the calibrator. During the survey, the meter was set for slow response on the A-weighted scale, as is standard for surveys of this type and as recommended by WHC-IP-0030, Section IH 1-10

The survey results are summarized in the following table. Both the "Ambient" and "During" levels noted are averages for the room/area, except in those cases where it was felt that a range more appropriately characterized the noise levels.

\begin{tabular}{||l|l|c|c|}
\hline ROOM \# & ROOM DESCRIPTION & $\begin{array}{c}\text { AMBIENT (PRE-ALARM) } \\
\text { NOISE LEVEL (dBA) }\end{array}$ & $\begin{array}{c}\text { (DURING) ALARM } \\
\text { NOISE LEVEL (dBA) }\end{array}$ \\
\hline 101 & shipping/receiving & 64 & 89 \\
\hline 102 & s \& $r$ office & (locked) & (locked) \\
\hline 103 & office & (locked) & (locked) \\
\hline 104 & NDE/NDA & 65 & 86 \\
\hline 107 & process area & 68 & 89 \\
\hline 109 & sample management & 62 & 100 \\
\hline 113 & process HVAC & 60 & 86 \\
\hline 116 & switchgear room & 70 & 90 \\
\hline 118 & mechanical room & 66 & 74 \\
\hline 120 & RPT room & 53 & (compressor OFF) \\
\hline 121 & storage & 55 & $\begin{array}{c}63 / 78 \\
\text { (compressor } 0 \text { F) }\end{array}$ \\
\hline
\end{tabular}




\begin{tabular}{|c|c|c|c|}
\hline ROOM \# & ROOM DESCRIPTION & $\begin{array}{l}\text { AMBIENT (PRE-A.LAR|M) } \\
\text { NOISE LEVEL (CEA) }\end{array}$ & $\begin{array}{l}\text { (OURING) ALAFN } \\
\text { NOISE LEVEL (dBA) }\end{array}$ \\
\hline 122 & RCD management & 51 & 95 \\
\hline 123 & operations mgr & 51 & 97 \\
\hline 125 & secretarial & 61 & 85 \\
\hline 126 & reception/waiting & 63 & 96 \\
\hline $128 / 129$ & conf/lunch room & 58 & 96 \\
\hline 124 & RPT & 51 & 100 \\
\hline 131 & soiled laundry & 53 & 65 \\
\hline 131 & clean laundry & 52 & 66 \\
\hline 134 & storage & 52 & 74 \\
\hline 136 & storage & 52 & 69 \\
\hline 139 & equip. maint. & 51 & 94 \\
\hline $140 / 141$ & women's locker & 59 & $85-93$ \\
\hline 144 & women's SWP/PCM & 57 & 54 \\
\hline 146 & men's SWP/PCM & 60 & 67 \\
\hline $148 / 150$ & men's Tocker & 61 & 85-1owest \\
\hline 149 & men's shower & 64 & 86 \\
\hline 152 & material prep. & 55 & 93 \\
\hline $\begin{array}{l}\text { North } \\
\text { stairwell }\end{array}$ & $-\cdots-\cdots$ & 50 & 65 \\
\hline $\begin{array}{l}\text { South } \\
\text { stairwell }\end{array}$ & --------- & 57 & 71 \\
\hline 201 & control room & 60 & 78 \\
\hline $201 \mathrm{~A}$ & control office & 57 & 89 \\
\hline 202 & computer room & 59 & 65 \\
\hline 203 & mechanical room & 55 & $85-92$ \\
\hline
\end{tabular}

In five of the rooms/areas where noise levels were measured both pre- and duringalarms -- 118 (mechanical room), 121 (storage room), 144 (women's SWP/PCM room), 146 (men's SWP/PCM room) and 202 (computer room), -- the sound levels measured during the alarm were less $10 \mathrm{dBA}$ higher than the "ambient" (pre-alarm) levels. In the remainder of the rooms/areas, the noise level difference was at least the $10 \mathrm{dBA}$ difference specified in the WRAP 1 FDC, SDC 7.8.

It is important to note that the "ambient" levels observed were those present at the time of the survey and are not necessarily indicative of what sound levels will be when the facility is operational and/or occupied.

If you have any questions, please call me at $372-3932$ or stop by to see me. 
Name of Prutected property: KFH-5366-WRAP

Cerrificate of Cumpletion

Address: 23rd \& Dayton, 200 West Area, Richland, WA 99352

Rep. of Prorected Prop. (name/phone): ICF Kaiser-Hanford Co.

Auchoricy Having Jurisdiction: Hanford Fire Chief

Address/Phone Number:

1. Type(s) of System or Service:

NFPA 72 . Chapeer 9 - Local

If alarm is transmitted to locacion(s) off premise, list where received:

NEPA 72. Chapter 9 - Emcrgency Voice/AJam Service Quantity of vice/alarm channels:

Quancity of speakers installed:

Single:

Quancity of tilephones or relephone jacks included insystem:

Multiple:

NFPA 72 , Chapter 4 - Auxiliary

Indicate type of connection:

Local energy. Shunt,

Locacion and telephone number for receipt of signals:

Parallel telephone

NFPA 72, Chapter 4 - Remote Station

Alarm:

Supervisory:

$X$ NFPA 72, Chapter 4 - Proprietary

If alarms are retransmitted to public fire service communications center or others, indicate location and telephone number of the or ganization receiving alarm:

Hanford Fire Station

Indicate how alarm is retransmitted:

KFAR Radio Transmitter

NFPA 72, Chapter $4-$ Central Station

The Prime Conrractor:

Central Stztion Location:

Mcans of transmission of signals from the protected premise to the central station:

MeCulloh

﹎ Digital Alarm Communieator Two-Way Radio

One-Way Radio

Others

Means of transmission of alams to the public fire service communications center:

l.

2 .

System Location:

Supplier

Organizacion Name/Phone

Plateau Electric

Representative Name/Phon:

Installer

3-1) Protection Systems
3-D Protection Systcms

R1chard Schneehagen (206) 881-5127

Service Organization

Denois Latt (503) 221-0299

Doug Perske (503) 22.1-0299

Figure 1-7.2.1 Cerideace of Compleciug.

[From NFPA 72 - 1990, 2-2.2 modified, and NFTA 71, 1.4.5 modihed] 
Electric Fire Punup:
e) _._. Fire I"ump Puner
1) — Firc Pump Running
g) — Phase Reversal
Engine-Driven Fire Pump:
h) _._Selector in Auto Posicim
i) _... Engine or Contol Panel Trouble
j) __ Fire Pump Runing
Engine-Driven Cenerator:
k) __ Selector in Auto Position
i) — Control Pancl Troublc
m) _- Transfer Switches
n) En_._. Engine Running
Other Supervisury Function(s) (specify):

6. Alarm Nouificacion Appliances and Circuics

Quanuty of indicating sppliance circuits connected to the system:

7

Types and quancites of alarm indicating appliances installed:
3) 16 Bclls
10 Inch
b) $\longrightarrow$ Horns
c) Chirnes
d) Other:
c) 19 Visual Sigrals Type: Strobe
f) _. Local Annunciator
with andible $\mathrm{X}$ w/o audible

i. Signaling Line Circuits:

Quancicy and Sryle (See NFPA 72, Table 3-6.1) of signaling line circuits connected to system:

Quaritity: Style:

8. System Power Supplies

a) Primary (Main)

Overcurrenc Procection:

Location:

Nominal Voltage: 120 V $\Lambda \mathrm{C}$

Type: CKT BREAKER

Current Rating. $3.5 \mathrm{AMP}$

b) Secondary (Suandby):

$\mathrm{X}$. Storage Baticry: Amp-Hour Rating 17.2 A/H

Colculated capncity to drive system, in hours: $-24-60$

Engine-driven generator dedicated to fire alarm system:

Location of fuel storage:

c) Emergency or Standby Systern used as backup to Primary Power Supply, instead of using a Secondary Power Supply:

Emergency System described in NFPA 70, Arricle 700

- Legally Required Standby System described in NFP. 70. Aricle 701

_._. Optional Scandby System described in NFPA 70. Arucle 702. Which also mees the performance requiremerits of Article 700 or 701

9. System Sofware

a) Operating System Software Revision Level(s):

b) Applicacion Software Revision Level(s):

c) Revision Completted by:

10. Comments:

(name) (firm)


Location of Recotd (As.Builh) Drawings:

3-D Protection Systems, 0110 S.W. Porter St., Fortland, 0R 97201

I.su:tion of Owners Matuals;

3-D Yrotection Systems

Locition of lest Repurts:

3-1 Protection Systems

A contracs, dated . dated , for lest and inspertion in accordance with NFPA statedard(s) No.(s) is in etticst.

2. Certificarism of System Installation

(Fill out after installation is complete and wiring checked for opcns, shorts, ground faults, and improper branching, hut prim tu (o) ducting operational acceptance tests.)

This system has been instulled in accordante with the NFHA standards as listed below, wits inspected by oir - includes the devices listred below and has bern in scrvice since

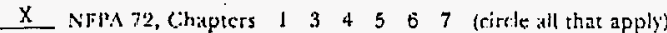

X- NFPA 70, Notionul Flectrical Comb, Article 760

- Manutocurer's Instructions

-... Other (specify):

Signed:

Organization: Plateau Electrical

3. Centuation ol System Operation

All operational features and fincrions of this system werc tested by

Date:

fouml a be operating properly in acordance with the requirements of:

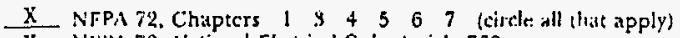

$\mathrm{X}$ NFPA 70, Natiunal Electrical Code, Articic 760

X Manufacturer's Instrustions

Oitier (speciti):

Signed:

Date:

Organizacion: 3-D Protection Systems

4. Alarm Initiuting Devices and (ircuits (Use blanks (1) indicate quantity of devices.)

M.NILAL

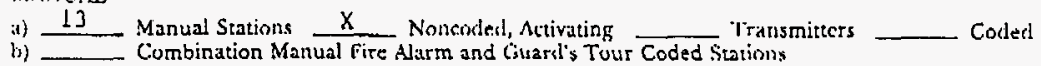

ALICOMAIT:

Coveringe:

Complete; _. Partial:
a) Smoke Detectors
b) T Dui: Detectors
c) 79 Heat Detector's
Ion
d) 3 Sprimkler iVater Flow Swiwhes: $\bar{X}$ Noncoded, attivating
Photo

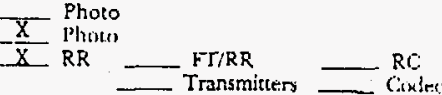

i) 1 Other (list): Ait Ssmpling Detector

5. Supervivory Signal Initiating Devices and Circuits (Use blanks to indicate quantity of devices.)

GLARD'S IOUK

a) ___ Coded Sucions

b) __ Nonceded Stations Activaling

c) Compulsory (iuard Tour System Cimprised of

Transmitters

Nuts: Combination devites rewrded under 4(b) and $5(a)$.

Tritnsmitter Stations and Intermedince Stationts

SPRINKLEKSYSIEM

a) _Coded Valve Supcrvisory Signaling Attaciments

b) __ Bulve Supcrvisory Switches Activasing __ _ Transmituen

c) - Site Wacer Temperature Foints

d) — Sice Witer Supply Level Points

Figure 1-7.2.1 Certificate of Completion, (cont)

[From NFIA 7n - 1990, 2-2.2 modified, and NFPA 7!, 1-4.4 modifie!! 
Location of Record (As.Bulle) Drawings:

3-D Protection Systems, 0110 S.W. Porter \$t., Portland, OR 97201

l.obilion of Owners Manuals:

3-D Protection Systems

Location of lest Repurts:

$3-D$ Protection Systems

$A$ coniser, dater , dacerl , for rest and inspection in accordance with NFPA standard(s) No.s.(s)

2. Certificarion of Systcm Installation

(Fill out after installalion is complete and wiring checked for opcus. shorts, ground faults, and improper branching. bus prior tu conducting operational atccptance tests.)

Thi, system has been installed in accordance with the NFHA standards as listed below, was inspected by on . intlus es the devices lisird below and has lomen in scivice since

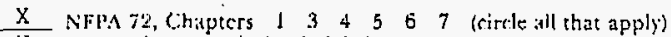

X- NFPA 70 , Natimul Electrical Coule, Articlo 760

$\mathrm{X}$ Manutacturer's Insmactions

Other (specify):

Signed:

Organization: Plategau Electrical

3. Cerrifualion of Sysecm Operation

All upcracional festures aull hunctions of this sysiem wacre tested by

fouml to be operating properly in atourdance with the requirentents of:

Date:

X NFPA 72. Chapters 1 \& 45667 (circle all (hat apply)

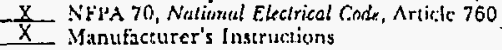
( Oiler (sjecify):

Signed:

Date:

Organizacion: $3-\mathrm{D}$ Procection Systems

4. Narm Initiating Devices and (Gircuits (Use blanks to indicate quantity of devices.)

MNILAL

i) 13 Menual Stations $\frac{X}{13}$ Noncoded, Activating Transmitters Coded

b) Combination Manual Fire Alarm and Guard's Tutr Coded Stotions

ALTOMATIC:

coverage:

Complete: $\mathrm{X}$ Partial:
i) 5 Smoke Detectors
b) $\frac{5}{79}$ Dus: Detectors
$\longrightarrow \operatorname{lom}$
Ion
(i) 79 Hcat Dctectors
TT
d) 3 Sprinkler Water Flow Swithes: $\frac{X}{1}$ Noncided, activating
Photo

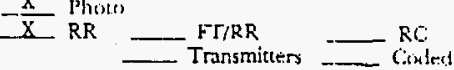

-) Lother (list): AIr Sampling Detector

5. Supervisory Signal Initiating Devices and Circuits (Use blanks to indicate quantity of devices.)

\section{GLARD'S IOOUR}

a) ___ Coded Scations

b) __ Nomcuded Stations Activating _______ Transmitcers

c) Compulsory Ciuard Tour System Cumpriscd of

Nute: Combination devices recorded under $4(\mathrm{~b})$ and $5(\mathrm{a})$.

Trinsmitter Statons amd Ititermediate Stationts

\section{SPRINKLEK SYSIEM}

i) Coded Valve Supcrvisory Sizmaling Atcaclumentw

bj Vulve Supcrvisory Switches Act

c) _L Site Wacer Temperature Points

d) _ Sile Wiater Supply Level Points

Figure [-7.2.1 Certificato of Completion, (cont)

[From NFl'A 78 - 1990, 2-2.2 moditied, and NFPA 71, 1.4.4 modiflis!] 



\section{DISTRIBUTION SHEET}

\begin{tabular}{|c|c|c|c|c|c|}
\hline \multirow{2}{*}{$\begin{array}{l}\text { To } \\
\text { Distribution }\end{array}$} & \multirow{2}{*}{\multicolumn{3}{|c|}{$\begin{array}{l}\text { From } \\
\text { WRAP } 1 \text { Facility }\end{array}$}} & \multicolumn{2}{|l|}{ Page 1 of 1} \\
\hline & & & & \multicolumn{2}{|c|}{ Date $12 / 12 / 96$} \\
\hline \multirow{2}{*}{\multicolumn{4}{|c|}{ Project Title/Work Order }} & \multicolumn{2}{|c|}{ EDT No. 161635} \\
\hline Project $W-026$ & & & & \multicolumn{2}{|l|}{ ECN No. } \\
\hline \multicolumn{2}{|c|}{ Name } & $\begin{array}{c}\text { Text } \\
\text { With All } \\
\text { Attach. }\end{array}$ & Text Only & $\begin{array}{l}\text { Attach./ } \\
\text { Appendix } \\
\text { Only }\end{array}$ & $\begin{array}{l}\text { EDT/ECN } \\
\text { Only }\end{array}$ \\
\hline
\end{tabular}

TL Watson

T4-02

X

JB Payne

T4-02

X

$\mathrm{KL}$ Humphrys

T4-52

X

RJ Bottenus

T4-52

$x$

JR McGee

T4-02

$X$

JK Kersten

T4-52 X

WRAP 1 DMC

T4-02 $X$ 
